\title{
Water Wells on St. Thomas, U.S. Virgin Islands
}

\section{By Judy I. Steiger and Richard Kessler}

\author{
U.S. GEOLOGICAL SURVEY
}

Open-File Data Report 91-504

Prepared in cooperation with the

U.S. VIRGIN ISLANDS WATER AND POWER AUTHORITY 


\section{U.S. DEPARTMENT OF THE INTERIOR}

BRUCE BABBITT, Secretary

U.S. GEOLOGICAL SURVEY

Dallas L. Peck, Director

For additional information write to:

District Chief

U.S. Geological Survey

P.O. Box 364424

San Juan, Puerto Rico 00936-4424
Copies of this report can be purchased from:

U.S. Geological Survey

Books and Open-File Reports Section

Federal Center, Box 25425

Denver, CO 80225-0425 


\section{CONTENTS}

Abstract

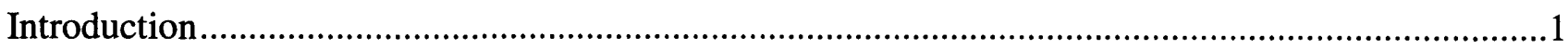

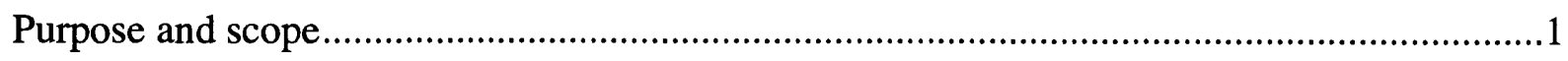

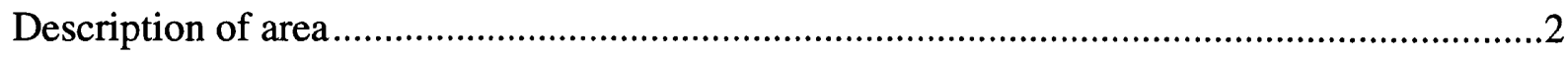

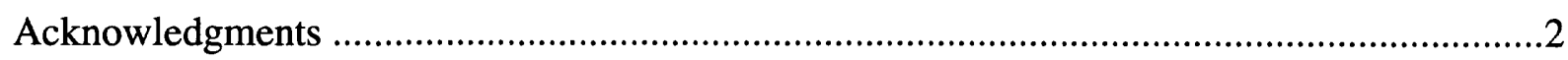

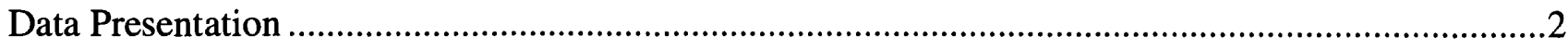

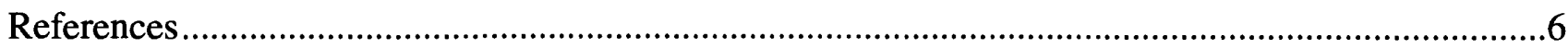

Appendix A - Well names, Estates, and site-identification

numbers of wells on St. Thomas, U.S. Virgin Islands................................................................

Appendix B - Lithologic descriptions and remarks for wells

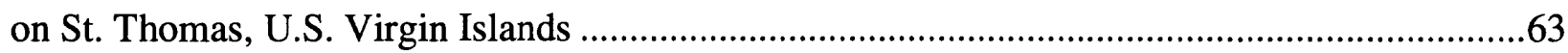

\section{Figures}

1 - 19. Maps showing:

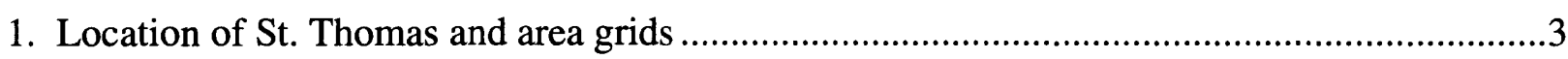

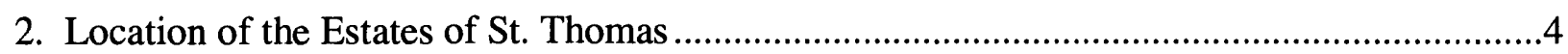

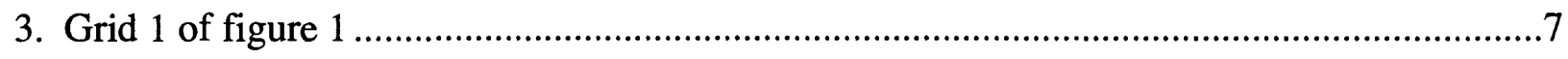

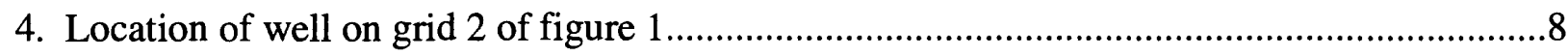

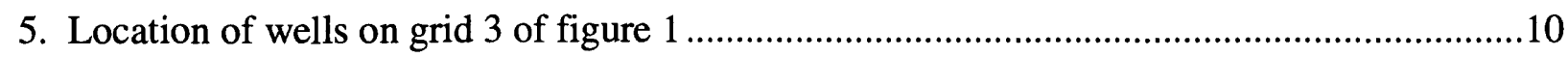

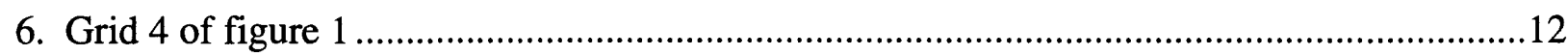

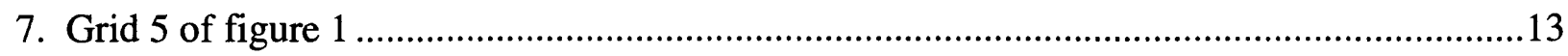

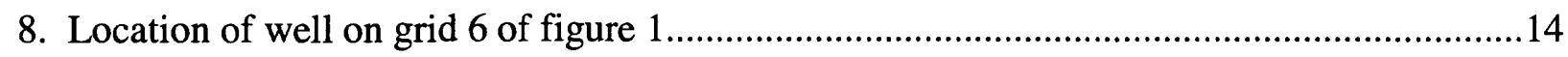

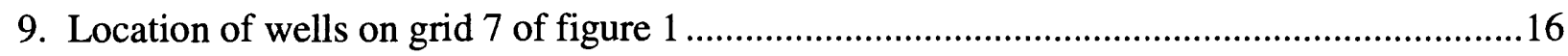

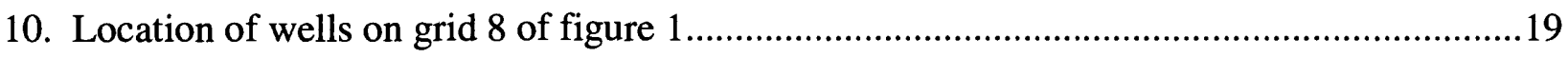

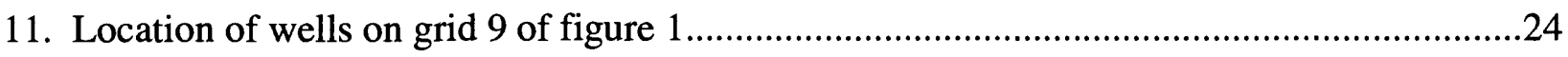

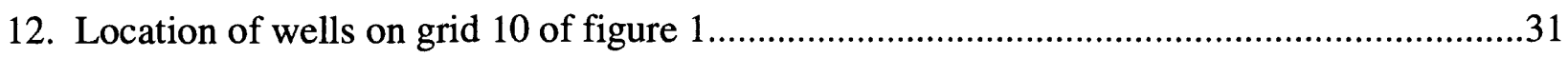

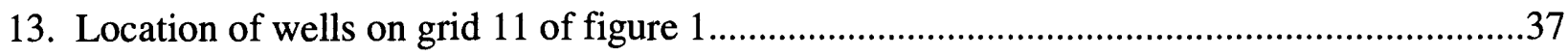

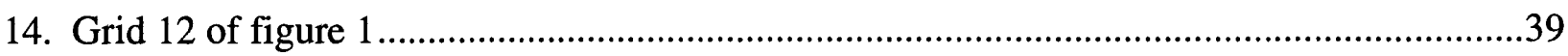

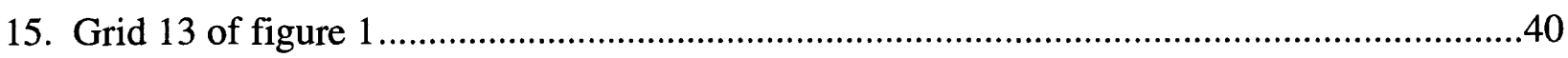

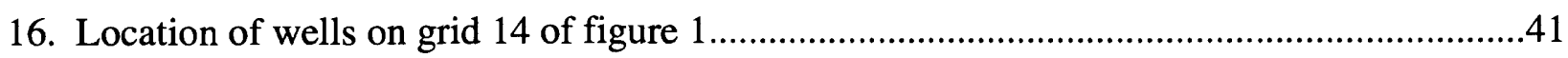

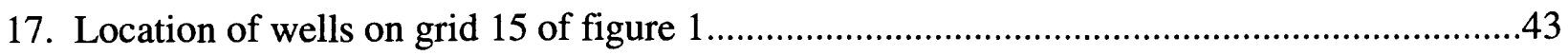

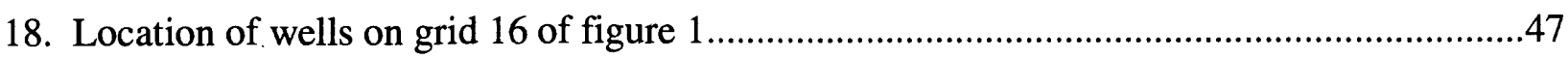

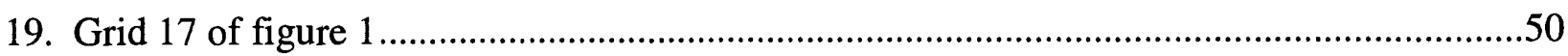




\section{Tables}

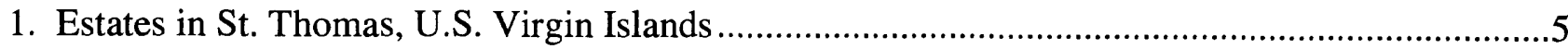

2 - 12. Description of:

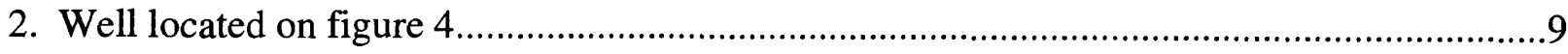

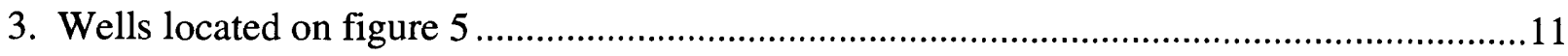

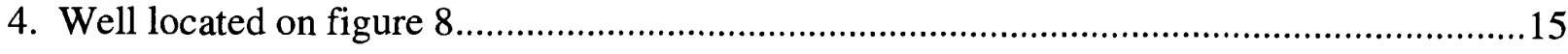

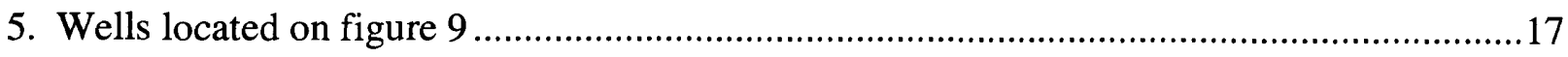

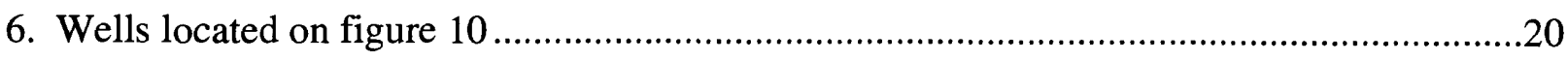

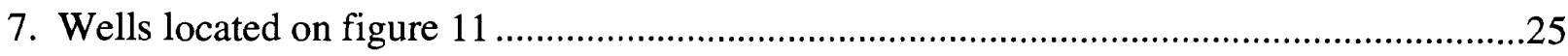

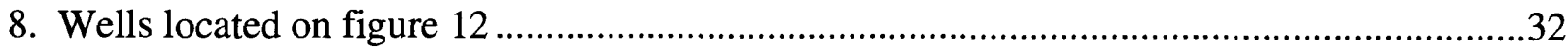

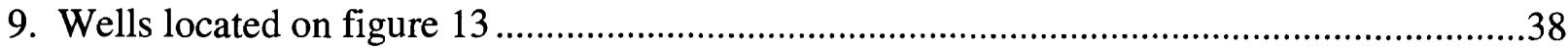

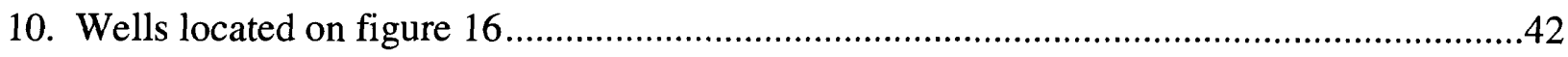

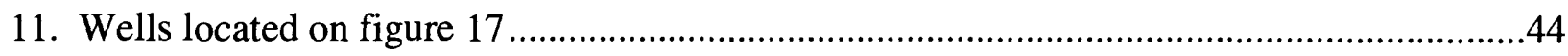

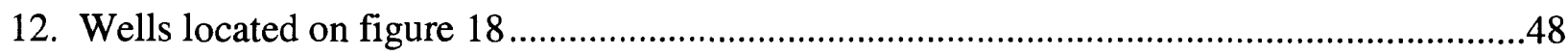

\section{CONVERSION FACTORS AND ACRONYMS}

\begin{tabular}{rll}
\hline Multiply & By & To obtain \\
\hline inch & 25.4 & millimeter \\
foot & 0.3048 & meter \\
mile & 1.609 & kilometer \\
square mile & 2.590 & square kilometer \\
gallon & 3.785 & liter \\
gallons per minute & 0.06308 & liter per second \\
gallons per day & 0.003785 & cubic meter per day \\
\hline
\end{tabular}

Acronyms used in this report:

U.S. Geological Survey (USGS)

U.S. Virgin Islands (USVI)

U.S. Virgin Islands Department of Planning and Natural Resources (VIDPNR)

U.S. Virgin Islands Water and Power Authority (VIWAPA) 


\title{
Water Wells on St. Thomas, U.S. Virgin Islands
}

\author{
By Judy I. Steiger and Richard Kessler
}

\begin{abstract}
This report presents a compilation of well-inventory data collected between December 1989 and December 1990 on St. Thomas, U.S. Virgin Islands. The report includes maps of well locations and tables of well-inventory data for 367 wells. The demand for freshwater in St. Thomas has exceeded the supply, because of the growth in population and tourism. Currently, the demand for water in St. Thomas is met by desalinated seawater, rainfall collected in cisterns, and ground water. The wellinventory data in this report provides a database to assist hydrologists, water managers, and planners in the development and full utilization of the ground-water resources of St. Thomas.
\end{abstract}

\section{INTRODUCTION}

Freshwater is a scarce and expensive commodity in the U.S. Virgin Islands (USVI) (Gómez-Gómez and others, 1985). To meet the demand for freshwater in St. Thomas, all available freshwater sources are used. In 1984, desalinated seawater provided about 71 percent of the freshwater supplies, and rooftop-rainfall catchments and ground water provided about 19 and 10 percent of the freshwater supplies respectively (Torres-Sierra and Dacosta, 1984).

The U.S. Virgin Islands Water and Power Authority (VIWAPA) is responsible for the supply and distribution of public water for the U.S. Virgin Islands. Because of increases in population and tourism, the development of the water resources of the USVI is becoming more important for the future of St. Thomas. In planning for long-term supply of water for the USVI, VIWAPA is considering all possible sources of water. It is anticipated that carefully planned ground-water development is the key to an inexpensive water supply in the future if ground-water contamination can be identified and controlled.

\section{Purpose and Scope}

The U.S. Geological Survey (USGS) entered into a cooperative program with VIWAPA during 1989 to complete a well inventory of St. Thomas, USVI. This report presents data for 367 wells including locations from the completed inventory conducted in St. Thomas between December 1989 and December 1990. 


\section{Description of Area}

St. Thomas is the second largest of the three principal islands (St. Croix, St. Thomas, and St. John) that comprise the U.S. Virgin Islands (fig. 1). The island is approximately 14 miles long and 2 to 3 miles wide and has an area of 32 square miles. The land surface is almost entirely sloping and extends seaward from a central ridge, which is 800 to 1,200 feet high and runs the length of the island. Flat land is confined to only a few small alluvium-filled embayments and parts of Estate Anna's Retreat and Estate Charlotte Amalie (fig. 2) in eastern St. Thomas (Jordan and Cosner, 1973).

\section{Acknowledgments}

The authors express their appreciation to the U.S. Virgin Islands Department of Planning and Natural Resources (VIDPNR), Division of Environmental Protection, for their assistance in helping to locate many of the wells on St. Thomas. Also, the cooperation of the many well and land owners who allowed access to their property and provided information about their wells is gratefully acknowledged.

\section{DATA PRESENTATION}

The locations and descriptions of 367 wells constructed before January 1991 on St. Thomas are presented in this report. For ease in locating wells, St. Thomas was divided into 17 grids (fig. 1). All grids shown in figure 1 are presented as separate figures in this report (figs. 3 through 19). Each grid represents a two-minute square section of the USGS 1982 photorevised, 7.5 minute series, topographic maps of St. Thomas. Each figure is published at the original topographic map scale of 1:24,000. Some grids cover areas in which no wells exist.

Grids that have wells located on them are followed immediately by a table containing selected well data (tables 2 through 12). When available, information presented in these tables includes: well number as referenced on the preceding figure, well name, primary use of water, year the well was constructed, reported depth of well, measured depth of well, well casing diameter, type of well finish and finish interval, landsurface altitude at well site, depth to water below land-surface datum, date water level measured, and yield of well. The well numbers used in tables 2 through 12 consist of two parts. The first part refers to the grid number from figure 1 , and the second part is a sequence number for the well within the grid. These numbers apply only to wells located on the accompanying figure. Identifiers mentioned in other reports can be identified by the site-identification numbers, which are presented in appendix A. The site-identification numbers conform with the established USGS Ground-Water Site Inventory classification scheme. The siteidentification number does not change and can be used to reference a specific well in other USGS publications.

The report also identifies the location of wells in terms of Estates in St. Thomas (fig. 2, table 1, and appendix A). Other information included in this report are the subsurface lithologic descriptions (when available) of a well site, and any special information about a well (appendix B). 

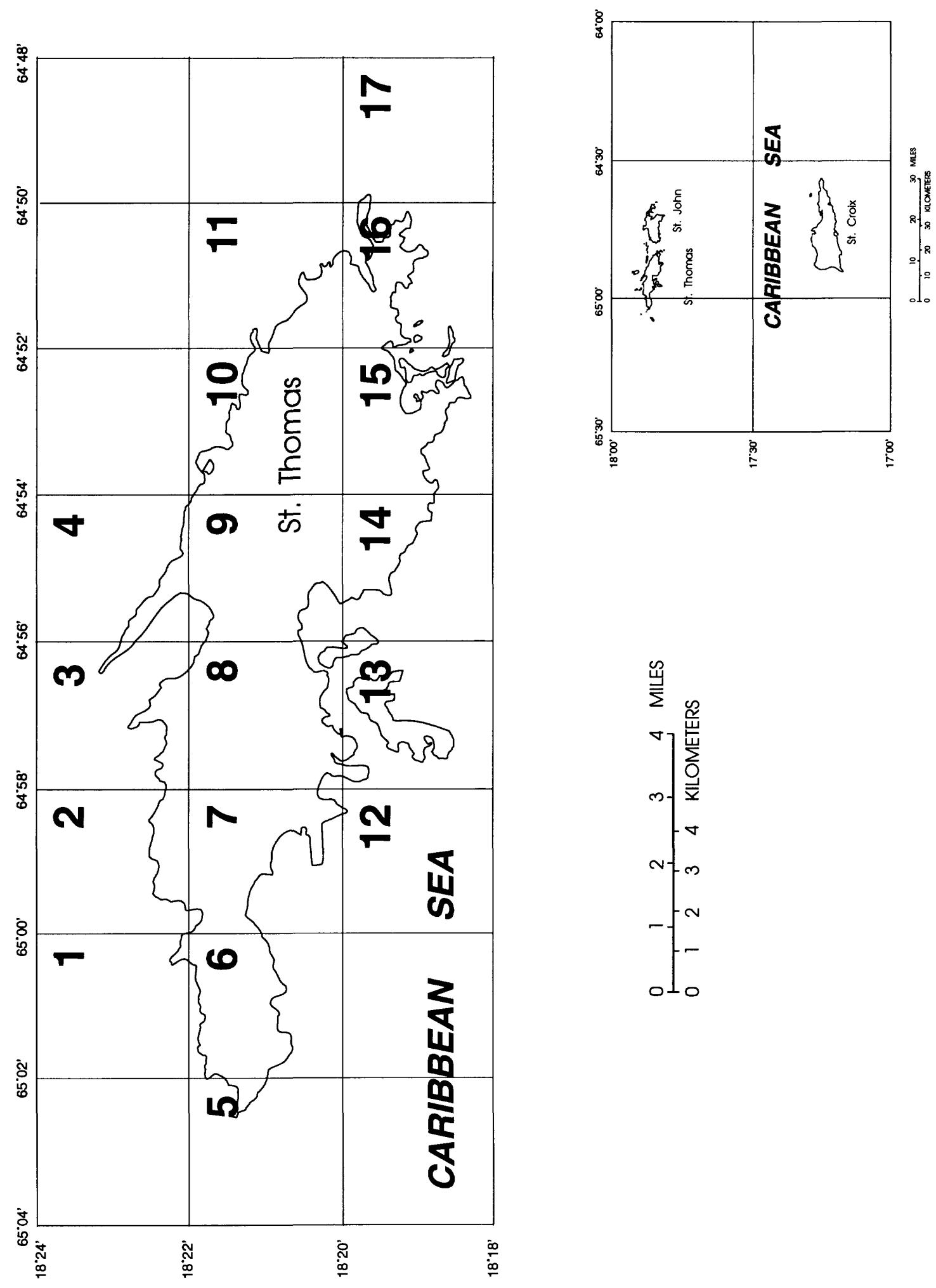

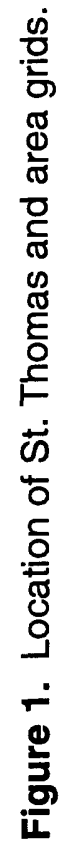



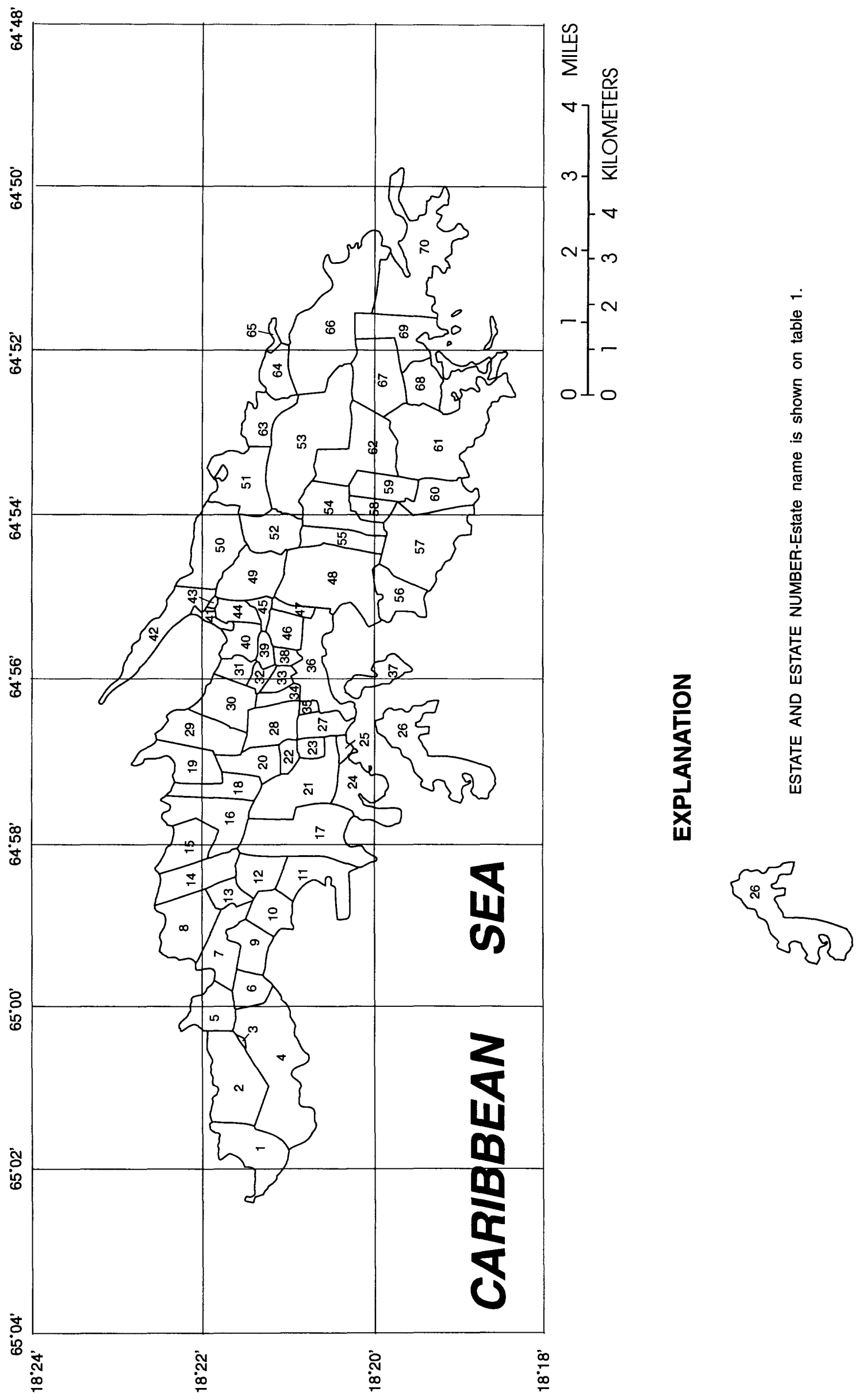

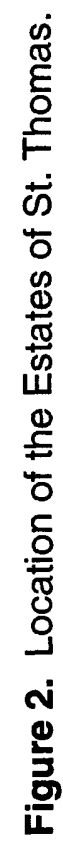


Table 1. Estates in St. Thomas, U.S. Virgin Islands

\begin{tabular}{|c|c|c|c|}
\hline $\begin{array}{l}\text { Estate' } \\
\text { number } \\
\text { (see } \\
\text { fig. 2) }\end{array}$ & $\begin{array}{l}\text { Estate } \\
\text { name }\end{array}$ & $\begin{array}{l}\text { Estate } \\
\text { number } \\
\text { (see } \\
\text { fig. 2) }\end{array}$ & $\begin{array}{l}\text { Estate } \\
\text { name }\end{array}$ \\
\hline 1 & Botany Bay & 36 & City of Charlotte Amalie \\
\hline 2 & Bordeaux & 37 & Hassel Island \\
\hline 3 & Bethesda & 38 & Litton's Fancy \\
\hline 4 & Fortuna & 39 & Mafolie \\
\hline 5 & Hope & 40 & Zurfriedenheit \\
\hline 6 & Perseverance & 41 & Magens Bay \\
\hline 7 & Santa Maria & 42 & Peterborg \\
\hline 8 & Sorgenfri & 43 & Herlein's Kob \\
\hline 9 & Bonne Esperance & 44 & Canaan and Sherpen Jewel \\
\hline 10 & Adelphi & 45 & Louisenhoj \\
\hline 11 & John Brewer's Bay & 46 & Hospital Ground \\
\hline 12 & Crown and Hawk & 47 & Ross \\
\hline 13 & Pearl & 48 & Thomas \\
\hline 14 & Caret Bay & 49 & St. Joseph and Rosendahl \\
\hline 15 & Neltjberg & 50 & Lovenlund \\
\hline 16 & Dorothea & 51 & Mandahl \\
\hline 17 & Lindberg Bay & 52 & Wintberg \\
\hline 18 & Bonne Resolution & 53 & Anna's Retreat (Tutu) \\
\hline 19 & Hull & 54 & Donoe \\
\hline 20 & Lilliendahl and Mariendahl & 55 & Raphune \\
\hline 21 & Contant & 56 & Bakkero \\
\hline 22 & Upper John Dunko & 57 & Frenchman Bay \\
\hline 23 & Lower John Dunko & 58 & New Herrnhut \\
\hline 24 & Nisky & 59 & Hoffman \\
\hline 25 & Crown Bay & 60 & Bolongo \\
\hline 26 & Water Island & 61 & Bovoni \\
\hline 27 & Altona & 62 & Charlotte Amalie \\
\hline 28 & Solberg & 63 & Tabor and Harmony \\
\hline 29 & St. Peter & 64 & Frydendahl \\
\hline 30 & Lerkenlund & 65 & Coki Point \\
\hline 31 & Misgunst & 66 & Smith Bay \\
\hline 32 & Elizabeth & 67 & Mariendahl \\
\hline 33 & Agnes' Fancy & 68 & Nadir \\
\hline 34 & Staabi & 69 & Frydenhoj \\
\hline 35 & Anna's Fancy & 70 & Nazareth \\
\hline
\end{tabular}

\footnotetext{
${ }^{1}$ The Estate number applies only to this report.
} 
Records of wells in St. Thomas were complied from the USGS historical files, VIDPNR files, previously published reports (Robison and others, 1973; Stevens and others, 1981; Graves and González, 1988), and verbal reports of well locations. These records were used to locate wells in the field. All the wells presented in this report have been verified and inventoried by USGS personnel.

Lithologic information and information about the construction of the wells was taken from well-drillers' logs, when available. Wells that could be sounded with a weighted steel tape were measured for depth by USGS personnel. Some of the information on well depths and type of well finish were provided by the well owner or were reported on previous well inventories.

Well casing diameters represent the inside diameter of the casing visible at land surface. In some instances, there may be a smaller diameter casing within the well. The smaller diameter casing is noted in the remarks section of the table.

Land-surface altitudes at the well site were estimated from USGS topographic maps. The contour interval of the topographic maps of St. Thomas is 40 feet. Twenty-foot contour intervals are shown where slopes are low. The accuracy of reporting land-surface altitudes in this report is considered to be half of the contour interval used.

Depth-to-water measurements were made by USGS personnel. Yields were reported by the driller or estimated by the well owner or well manager. Wells that were pumping and had in-line flow meters were measured for yield by USGS personnel.

\section{REFERENCES}

Gómez-Gómez, Fernando, Quiñones-Márquez, Ferdinand, and Zack, A.L., 1985, U.S. Virgin Islands groundwater resources -National Water Summary 1984: U.S. Geological Survey Water-Supply Paper 2275, p. 409-413.

Graves, R.P., and González, Ralph, 1988, Potentiometric surface of the Turpentine Run basin aquifer in the Tutu area, eastern St. Thomas, September 11, 1987: U.S. Geological Survey Water-Resources Investigations Report 88-4131, $1 \mathrm{pl}$.

Jordan, D.G., and Cosner, O.J., 1973, A survey of the water resources of St. Thomas, Virgin Islands: U.S. Geological Survey Open-File Report, 55 p.

Robison, T.M., and others, 1972, Water records of the U.S. Virgin Islands, 1962-69: U.S. Geological Survey Water Data Report, 163 p.

Stevens, K.E., Gómez-Gómez, Fernando, and Alicea, José, 1981, Water wells in the U.S. Virgin Islands, Part 1, St. Thomas: U.S. Geological Survey Open-File Report 82-82, 112 p.

Torres-Sierra, Heriberto, and Dacosta, Rafael, 1984, Estimated water use in St. Thomas, U.S. Virgin Islands, July 1983 - June 1984: U.S. Geological Survey Open-File Data Report 84-721, 1 pl. 


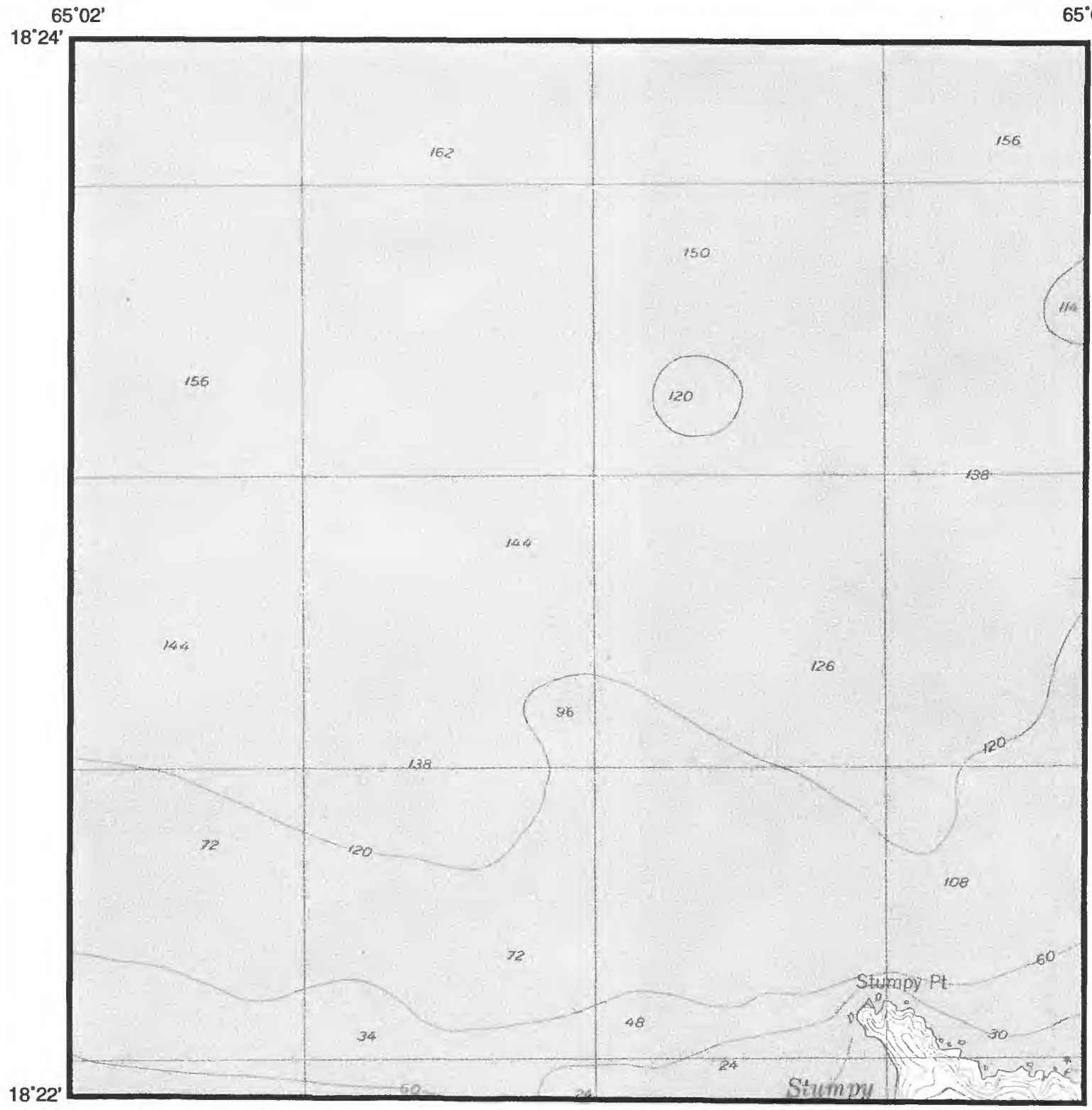

Base from U.S. Geological Survey

Westem St. Thomas, V.I. 1:24,000, 1982

SCALE

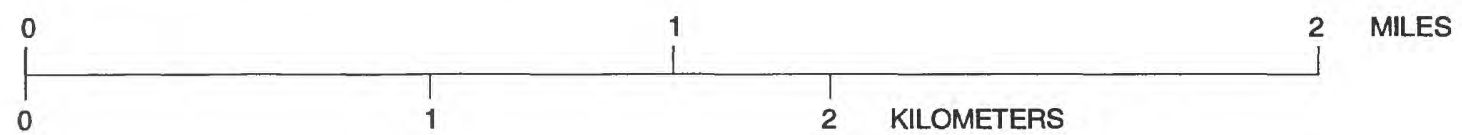

Figure 3. Grid 1 of figure 1. 


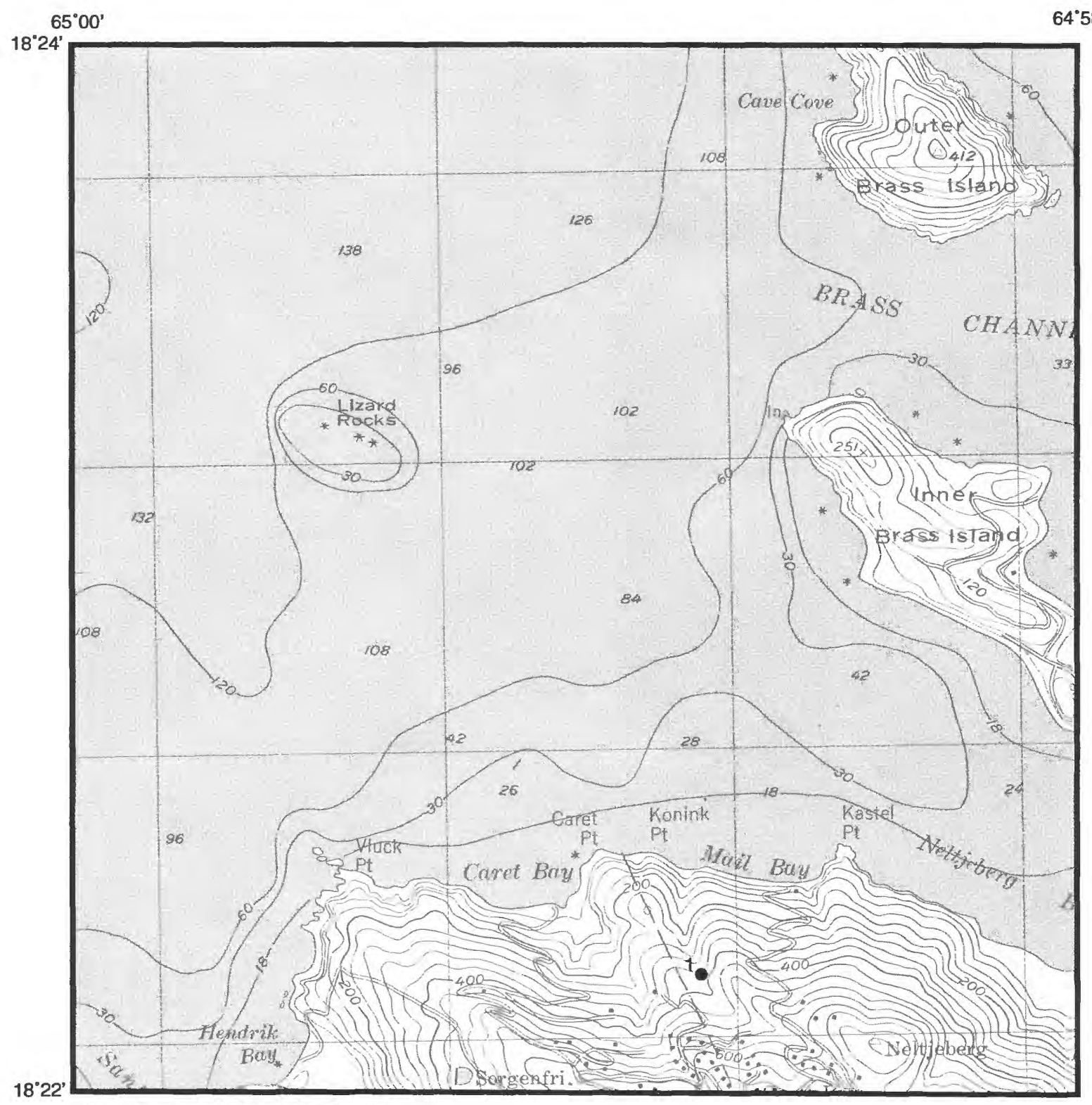

Base from U.S. Geological Survey

Central St. Thomas, v.l. 1:24,000, 1982 SCALE

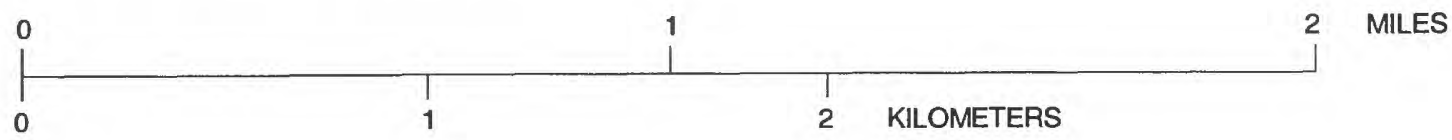

Figure 4. Location of well on grid 2 of figure 1. The well number shown on this figure corresponds to the well number which begins with 2- in table 2 and appendixes $A$ and $B$. 


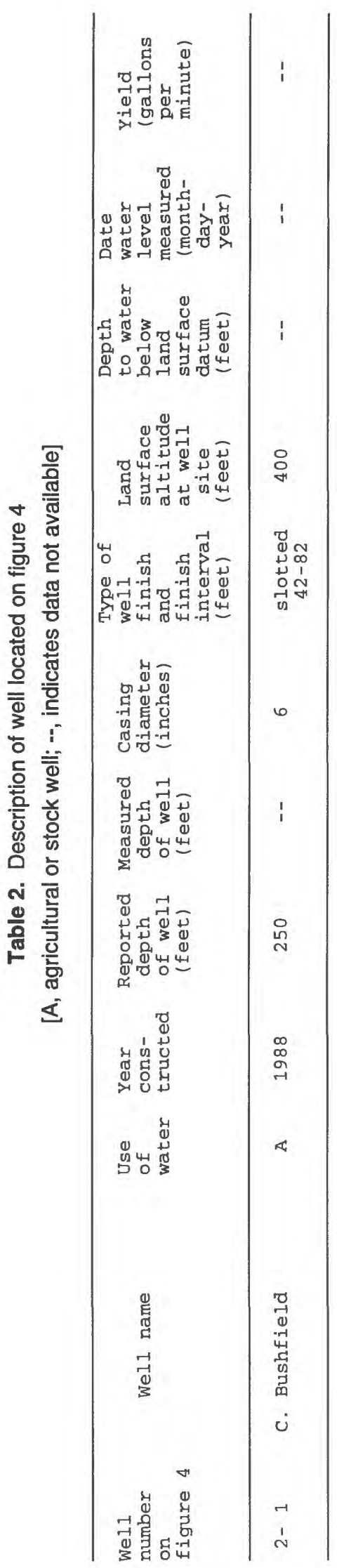




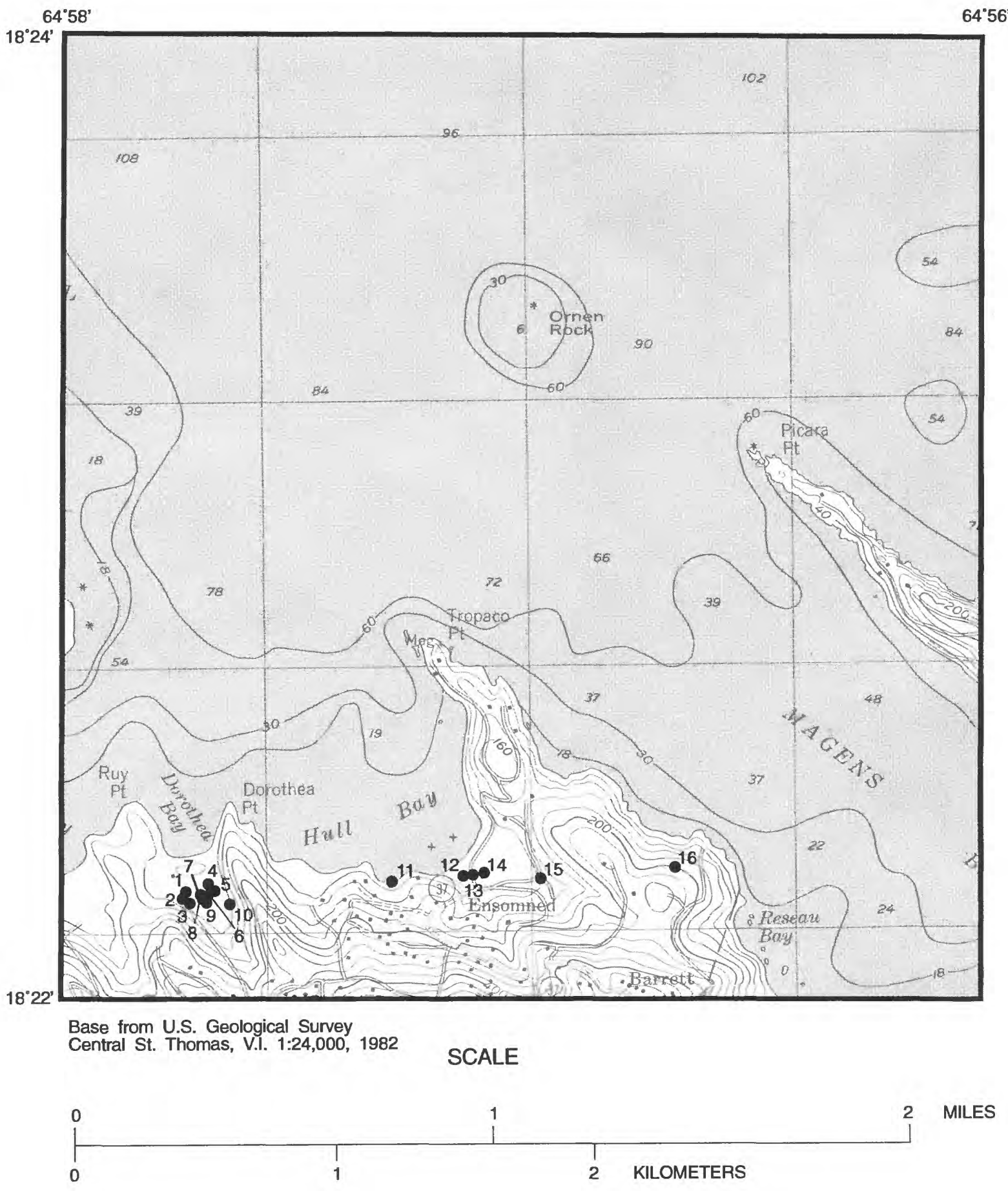

Figure 5. Location of wells on grid 3 of figure 1. The well numbers shown on this figure correspond to the well numbers which begin with 3 - in table 3 and appendixes A and B. 


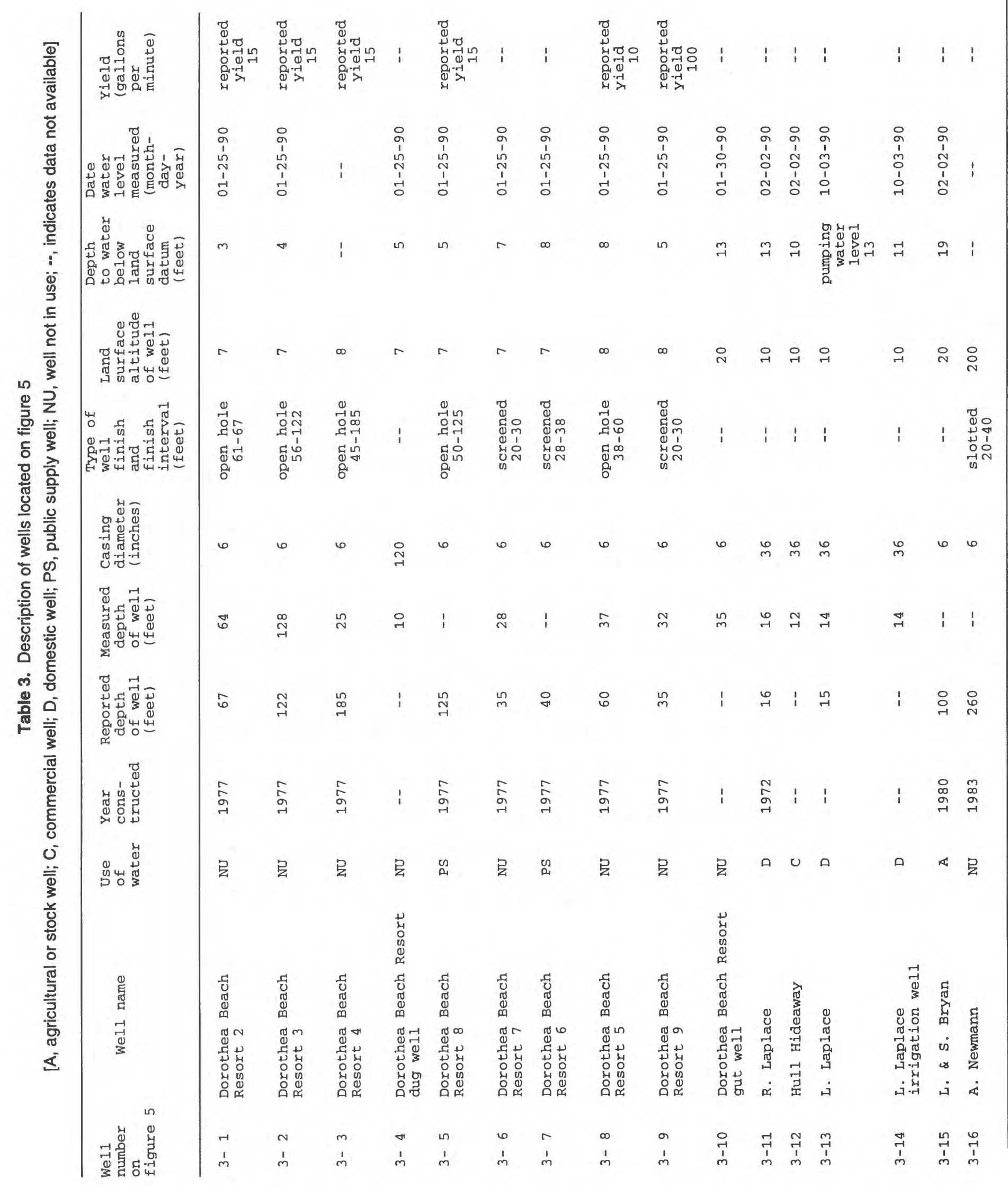



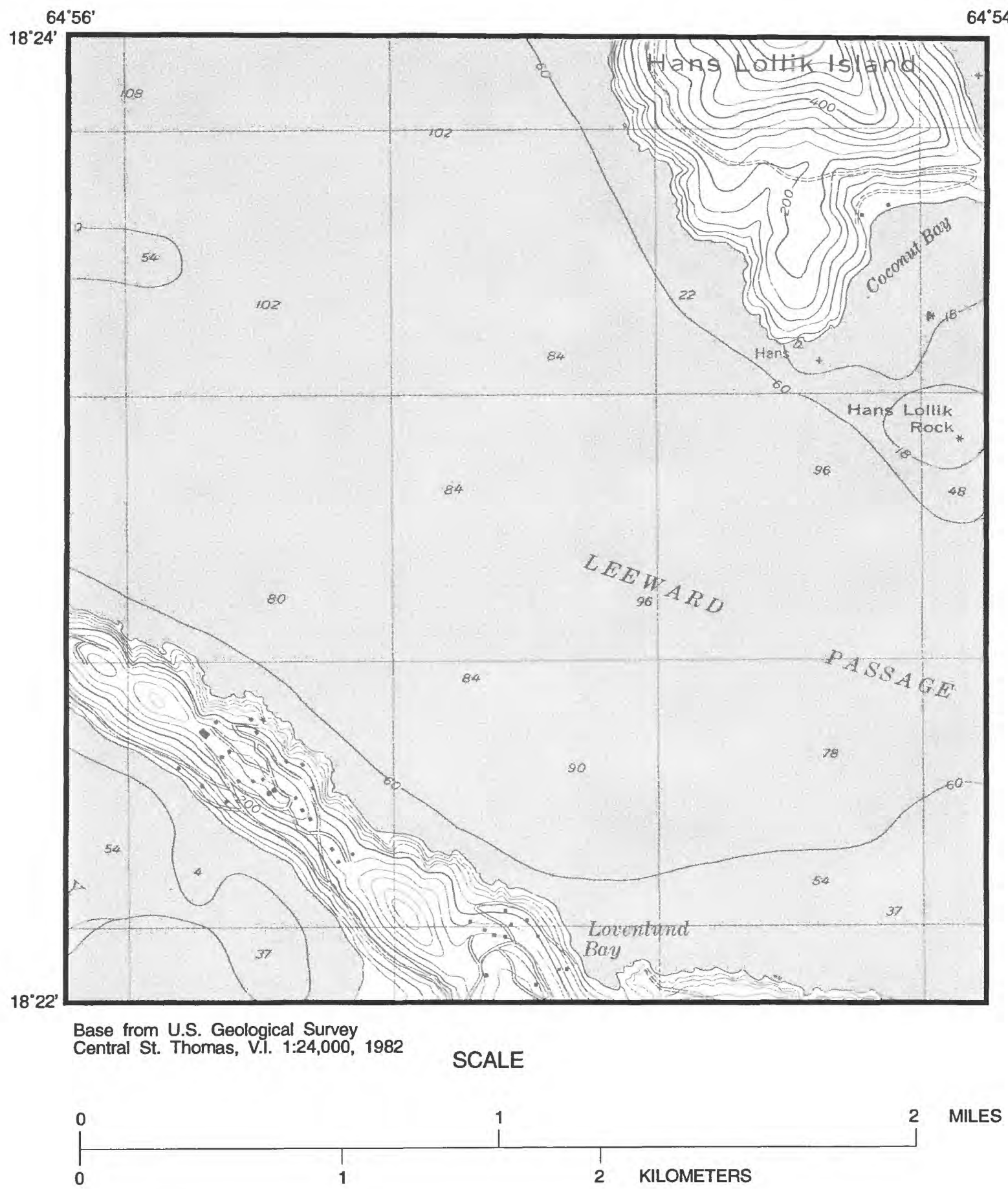

Figure 6. Grid 4 of figure 1. 

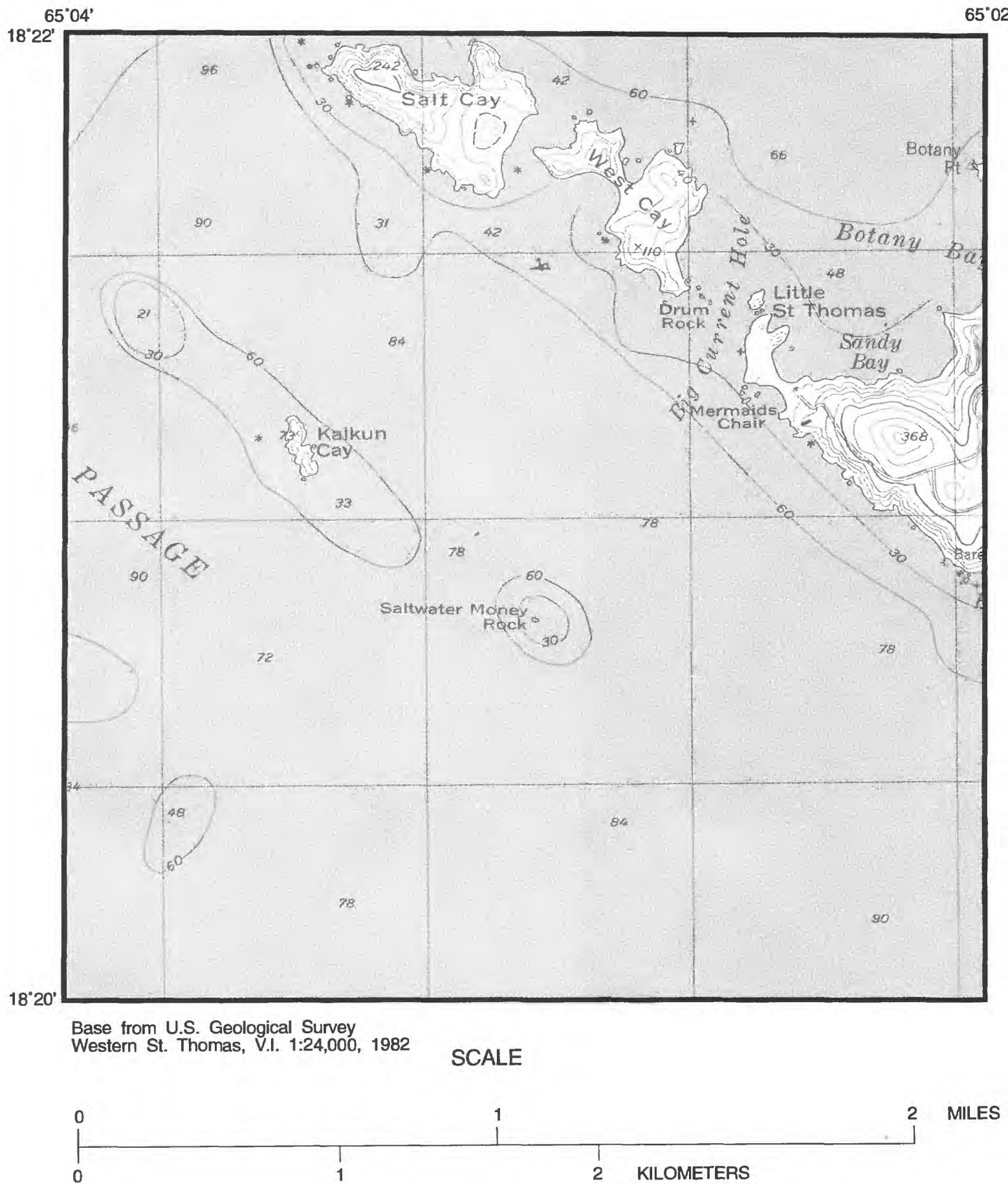

Figure 7. Grid 5 of figure 1. 


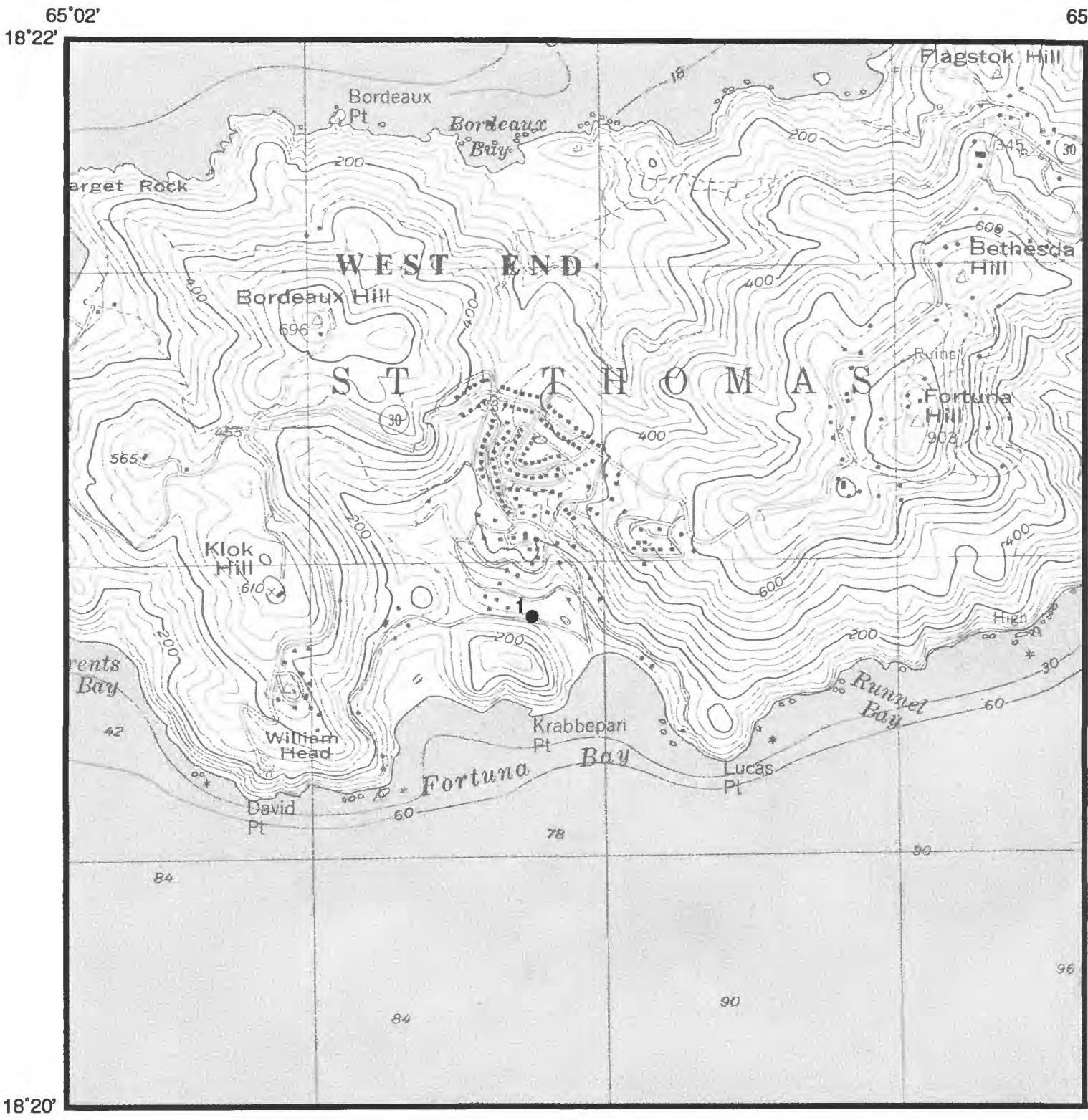

Base from U.S. Geological Survey Western St. Thomas, V.I. 1:24,000, 1982

SCALE

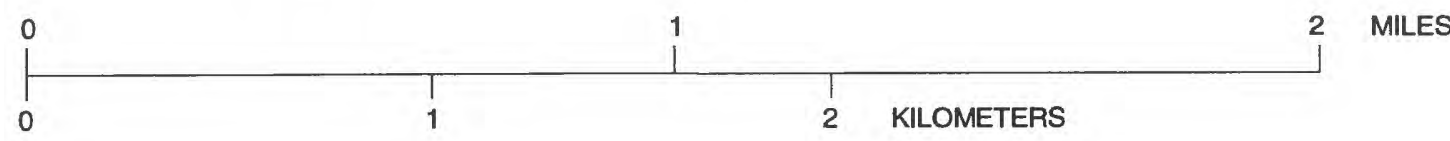

Figure 8. Location of well on grid 6 of figure 1 . The well number shown on this figure corresponds to the well number which begins with 6 - in table 4 and appendixes A and B. 


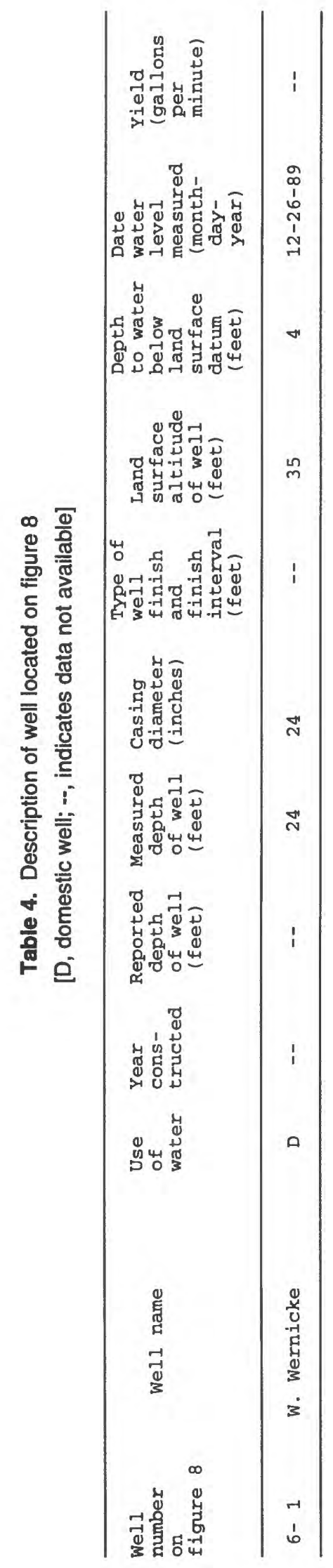




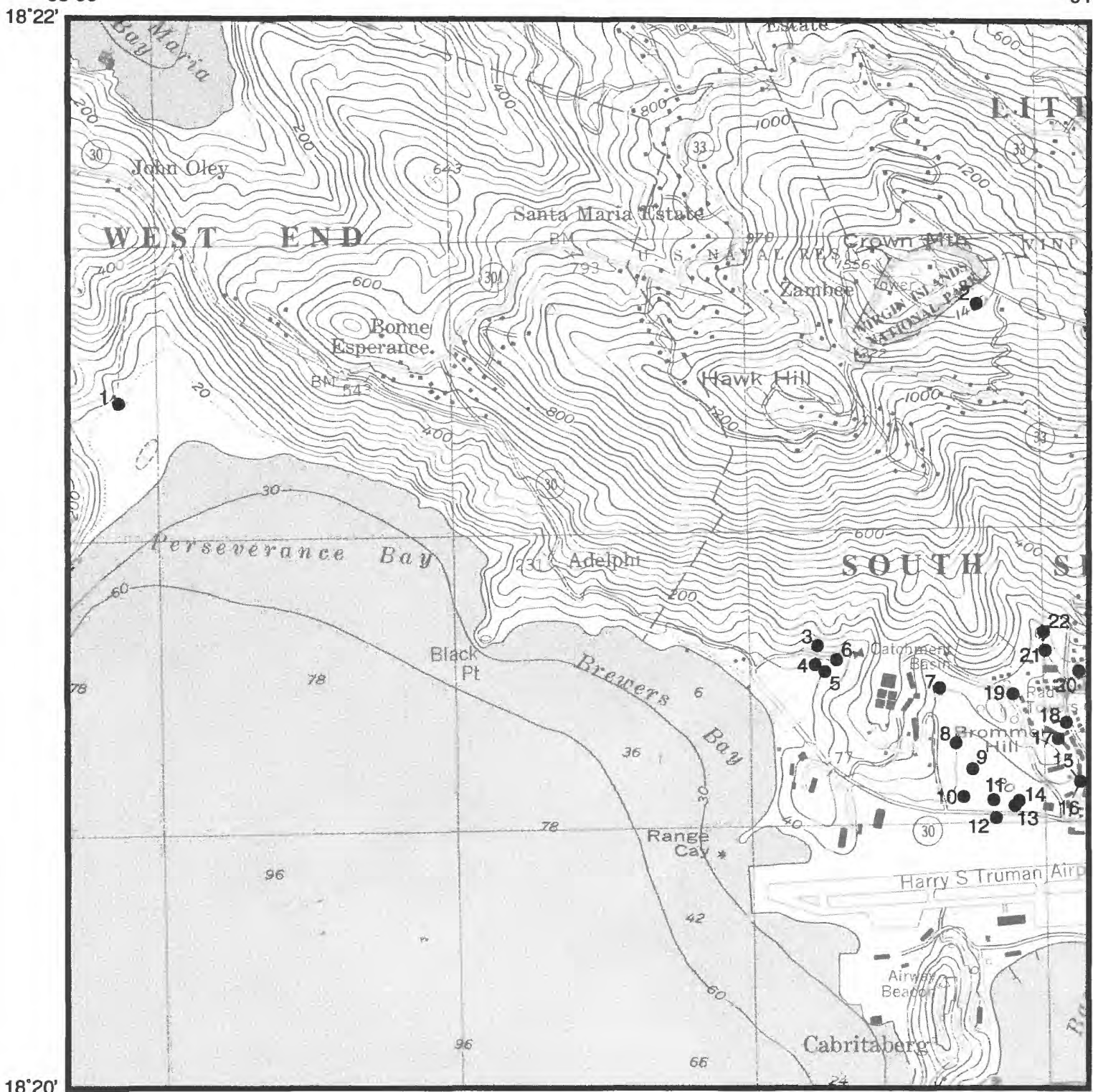

Base from U.S. Geological Survey

Central St. Thomas, v.1. 1:24,000, 1982

SCALE

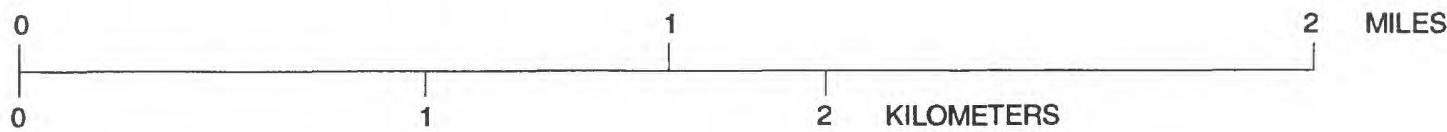

Figure 9. Location of wells on grid 7 of figure 1. The well numbers shown on this figure correspond to the well numbers which begin with 7 - in table 5 and appendixes $A$ and $B$. 


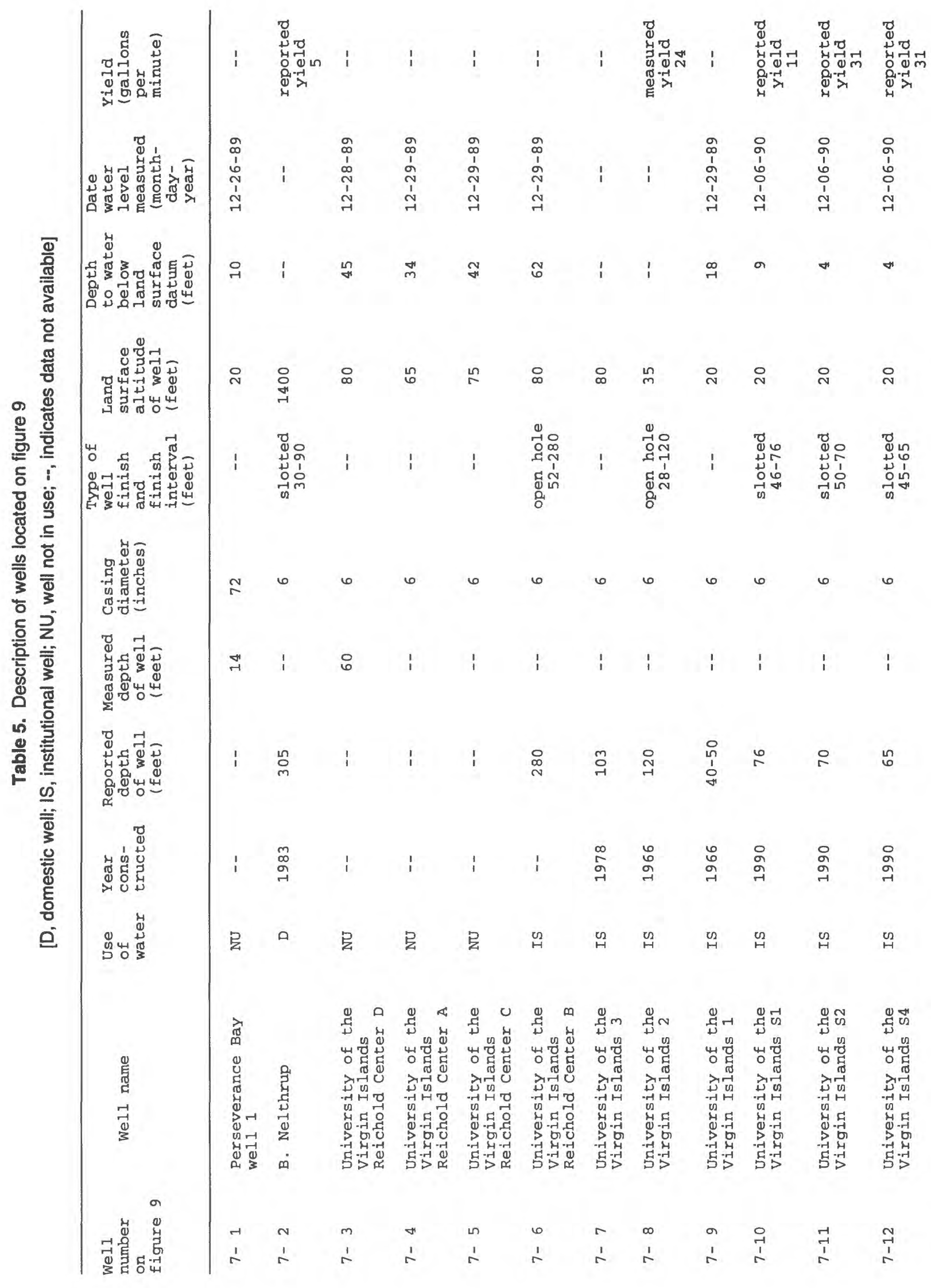




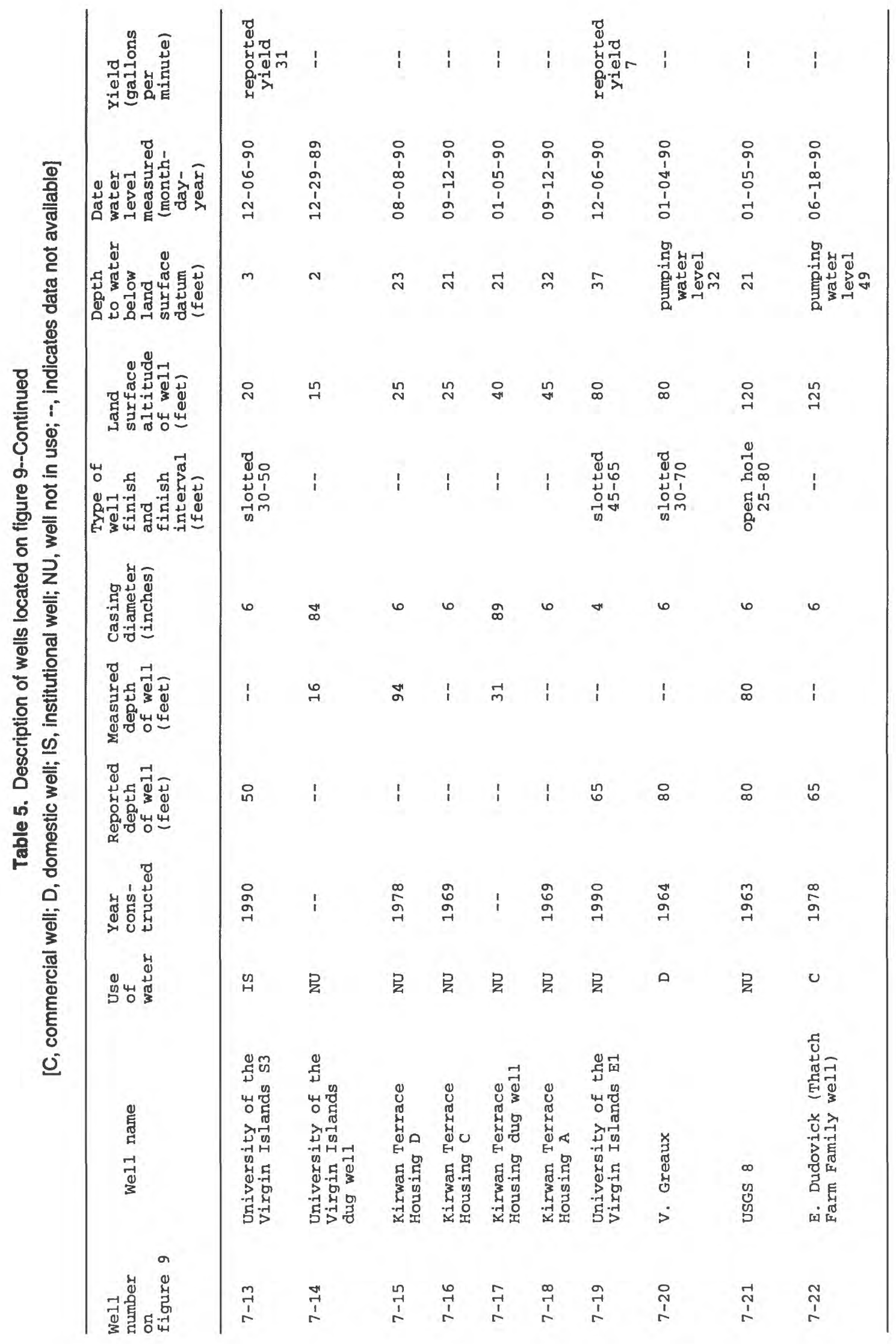




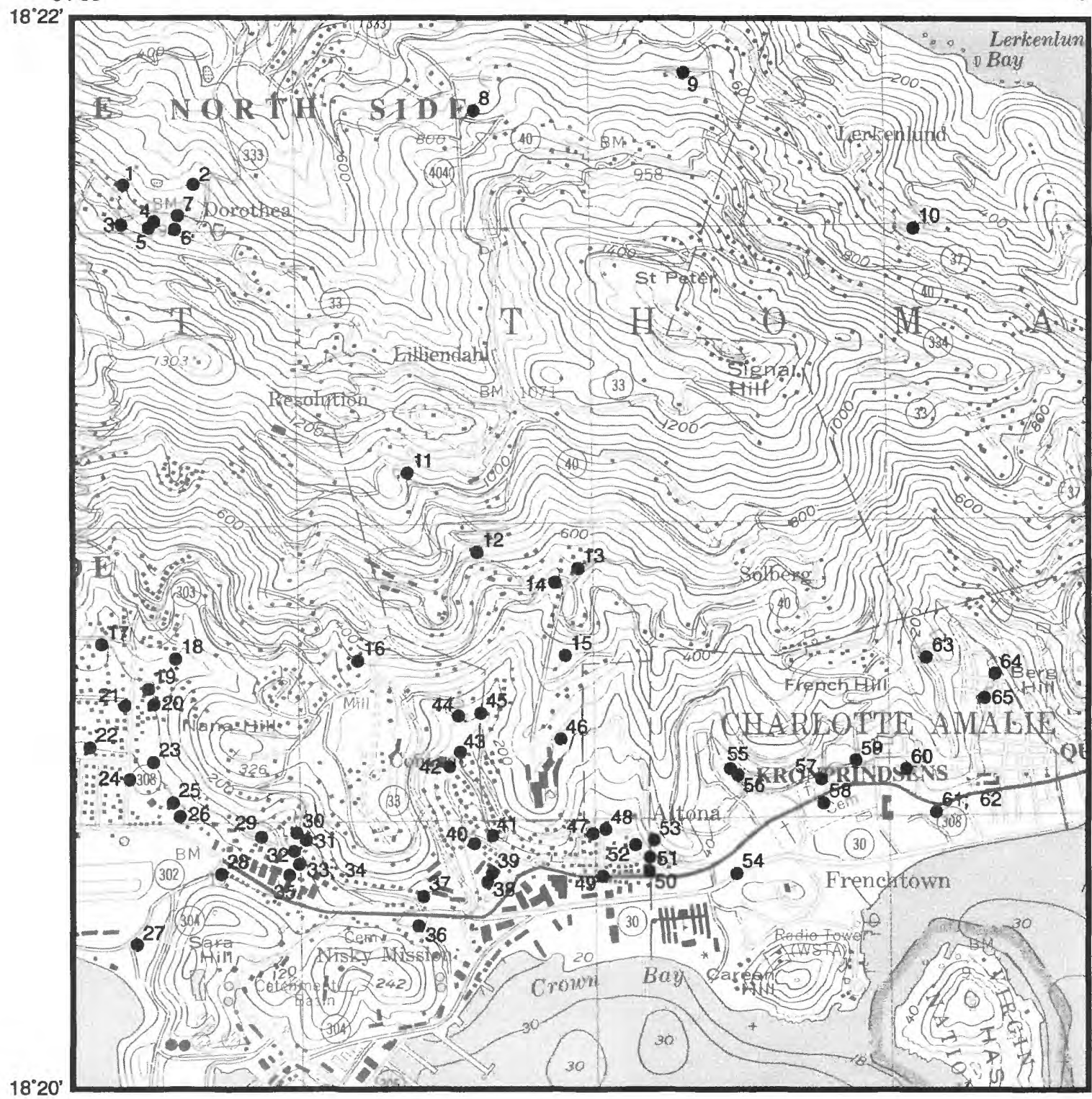

Base from U.S. Geological Survey Central St. Thomas, V.I. 1:24,000, 1982

SCALE

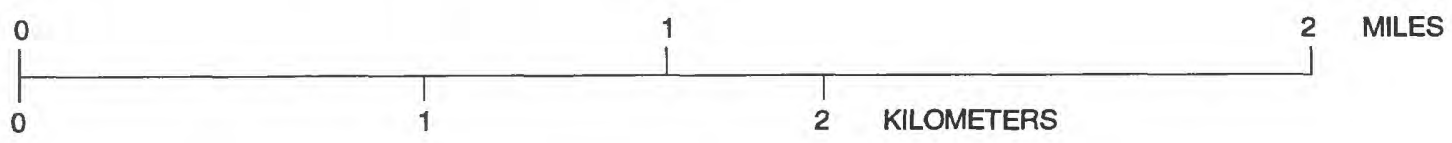

Figure 10. Location of wells on grid 8 of figure 1 . The well numbers shown on this figure correspond to the well numbers which begin with 8 - in table 6 and appendixes A and B. 


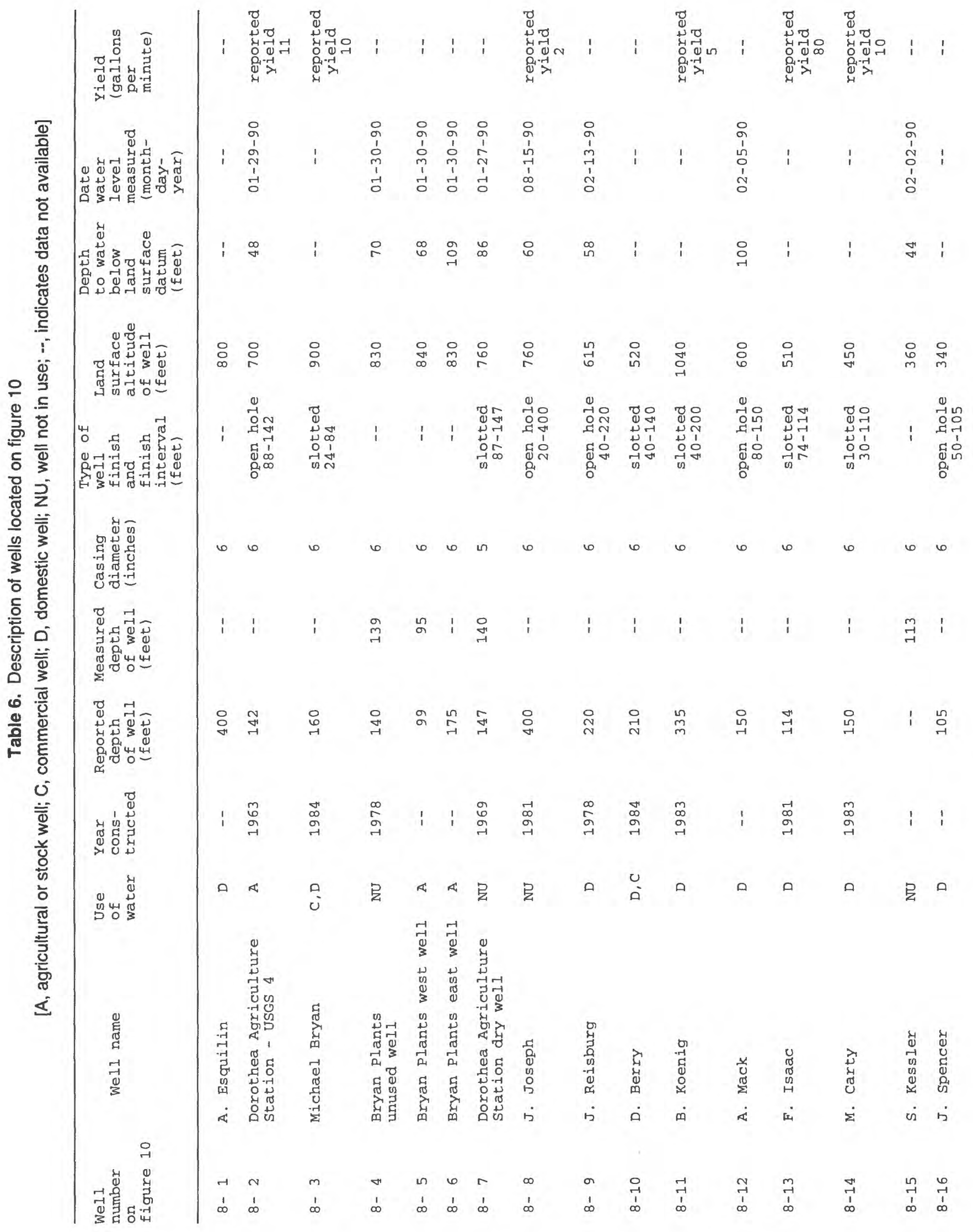




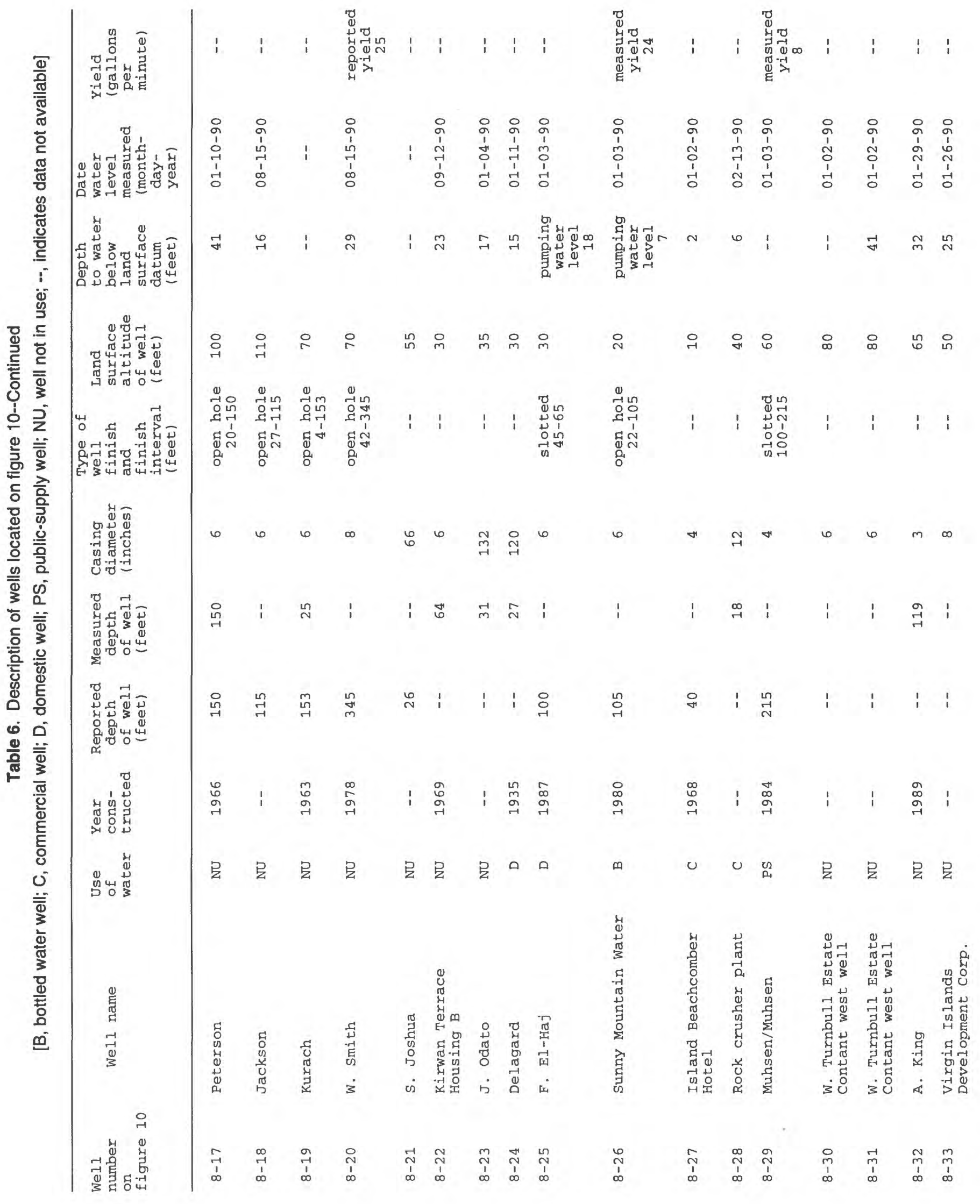




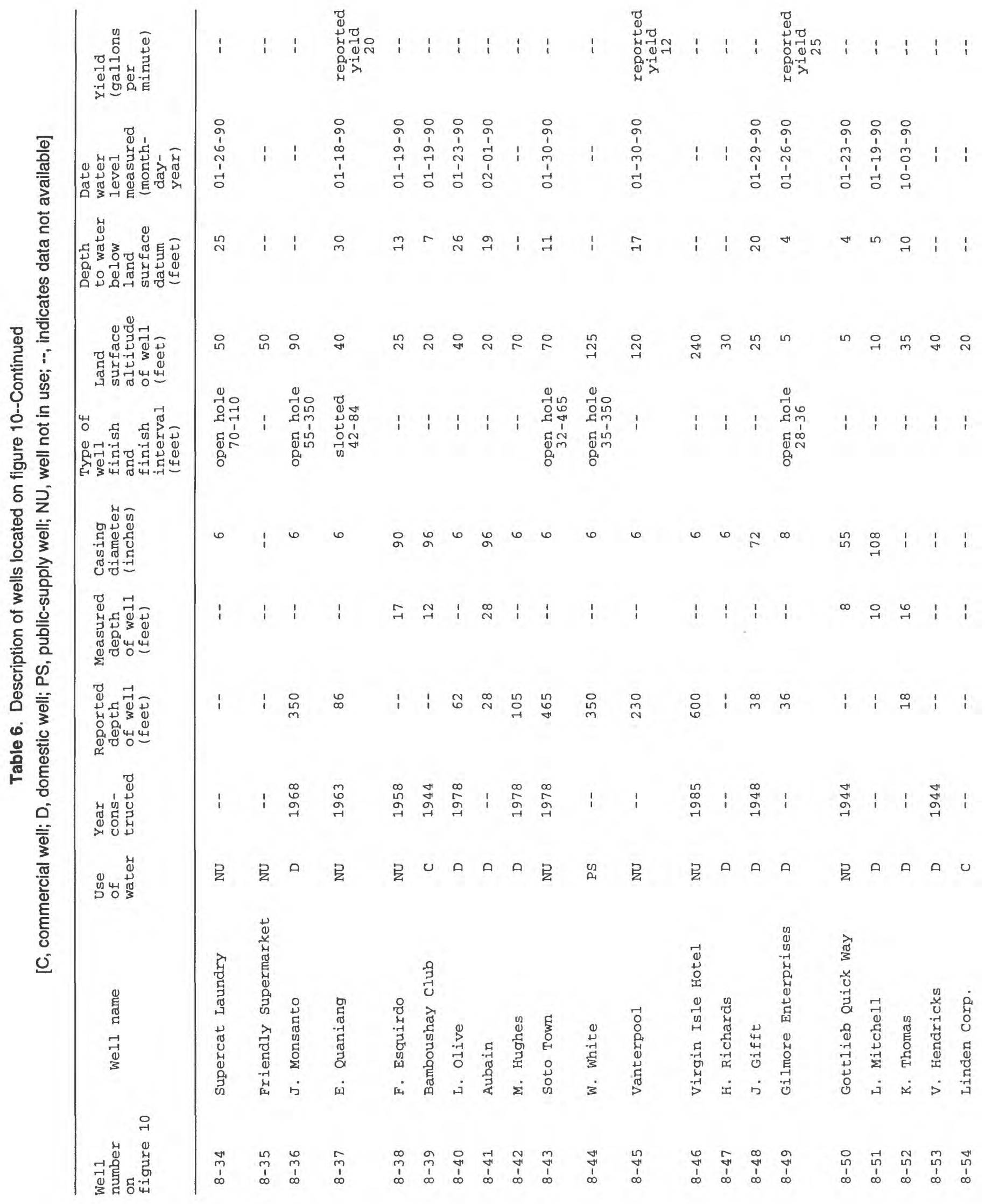




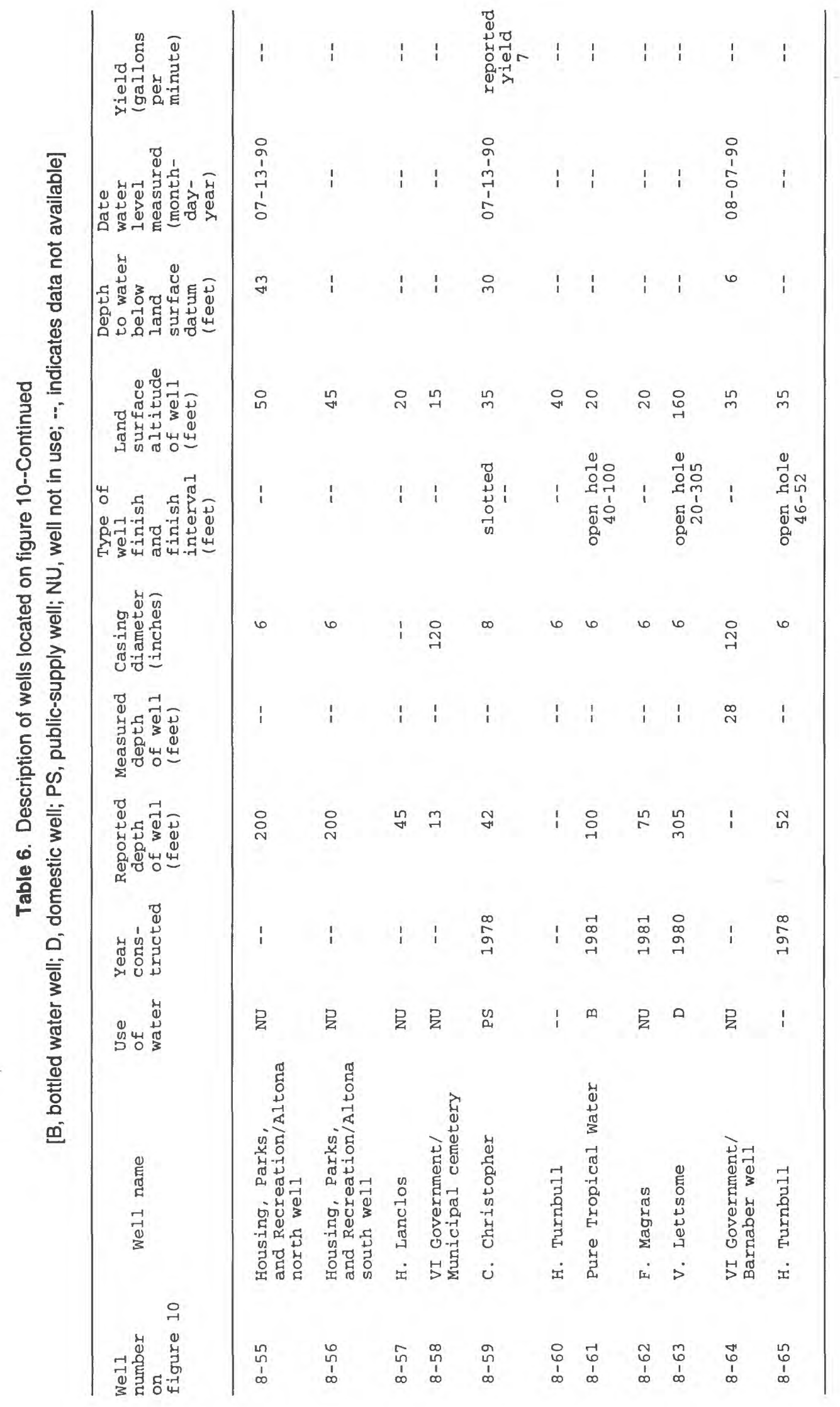




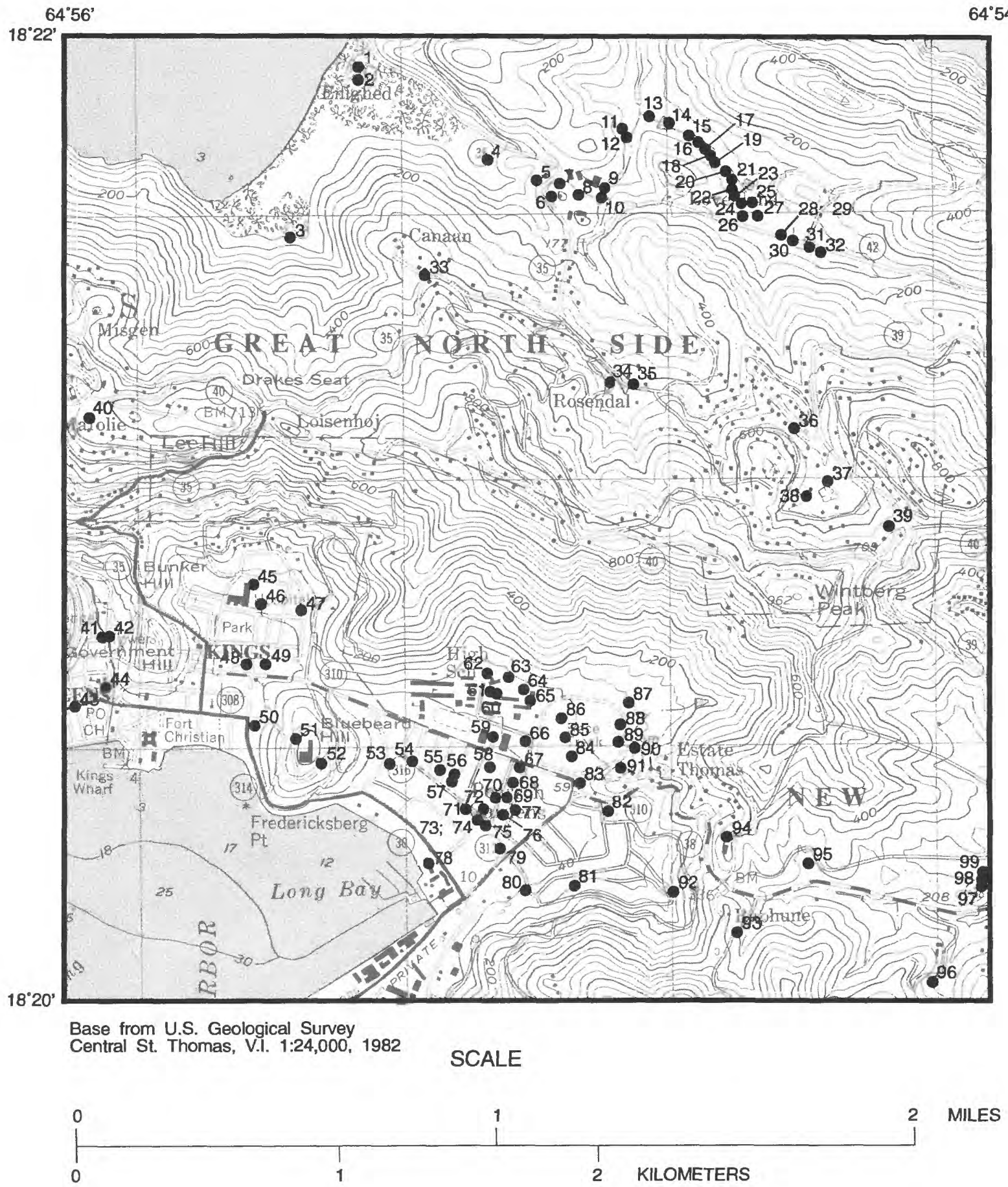

$64^{\circ} 54^{\prime}$

Figure 11. Location of wells on grid 9 of figure 1. The well numbers shown on this figure correspond to the well numbers which begin with 9 - in table 7 and appendixes $A$ and $B$. 


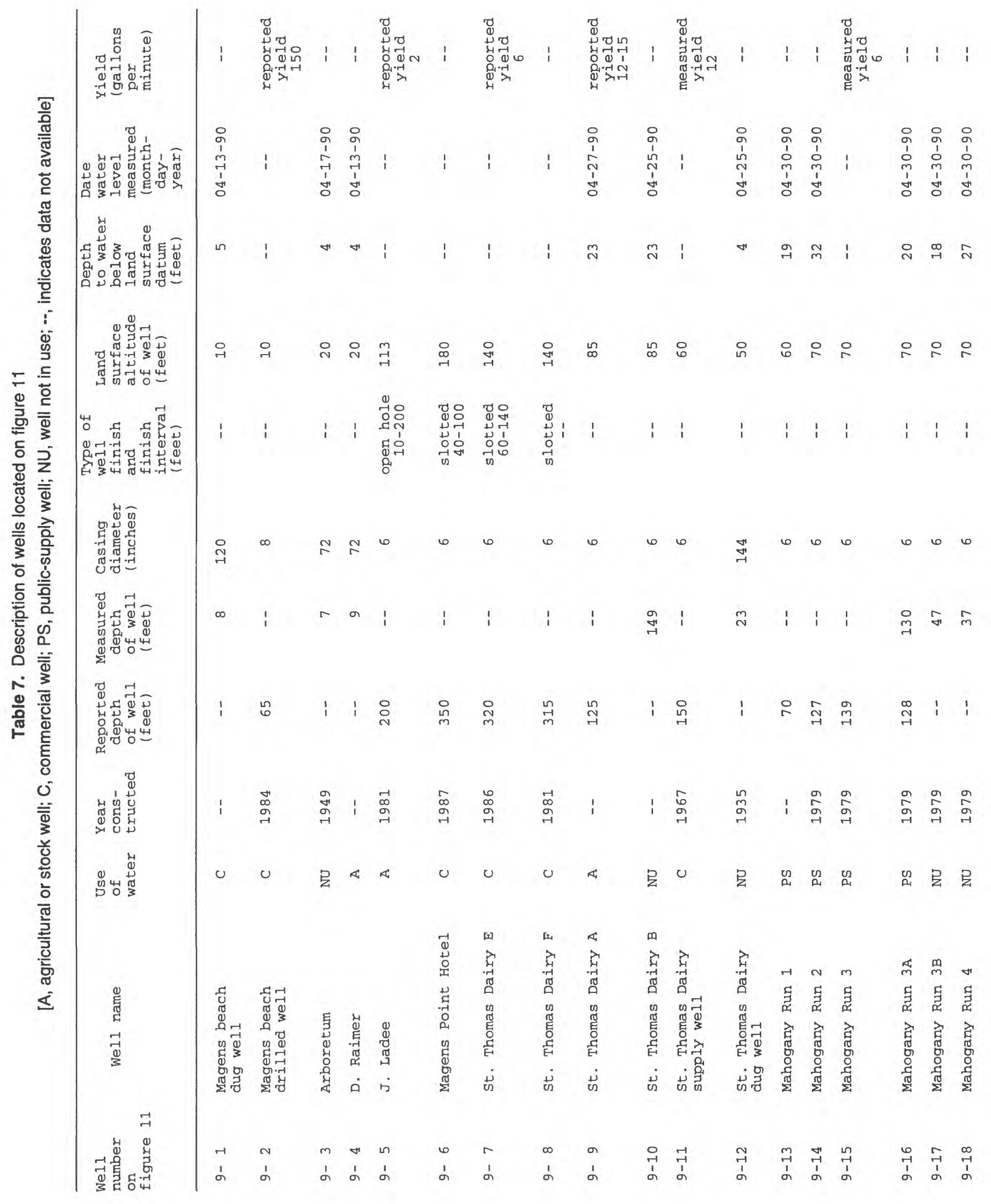




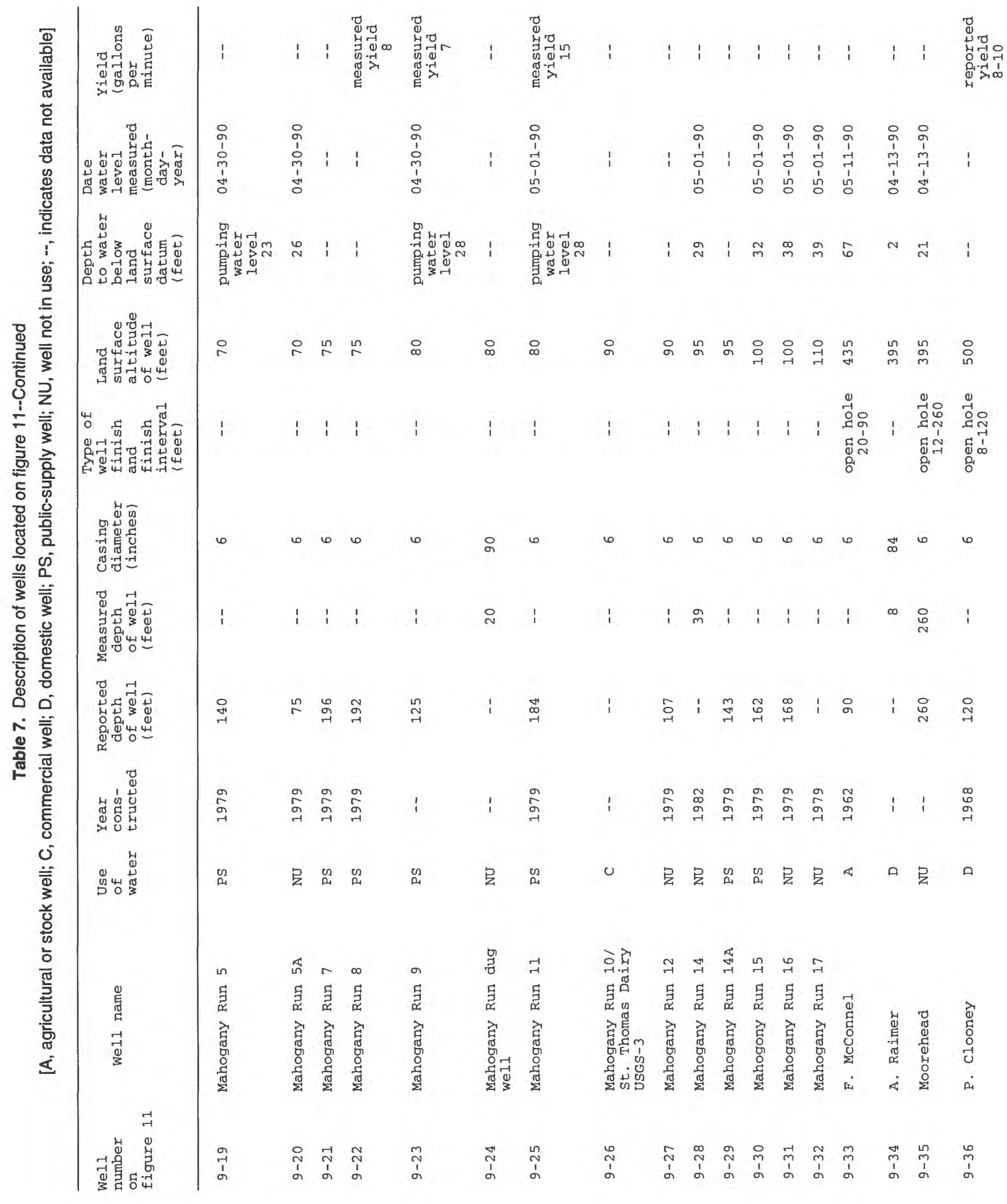




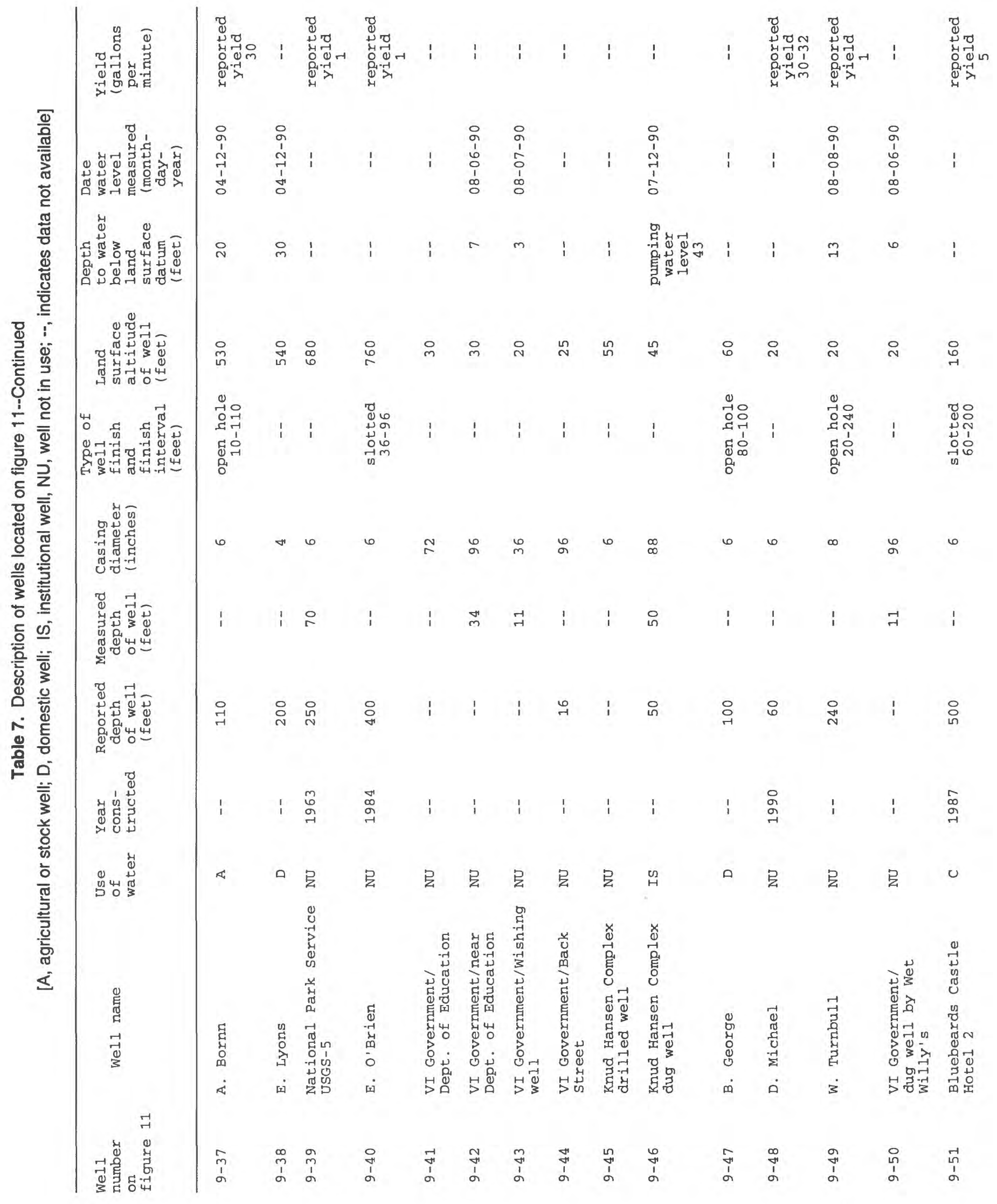




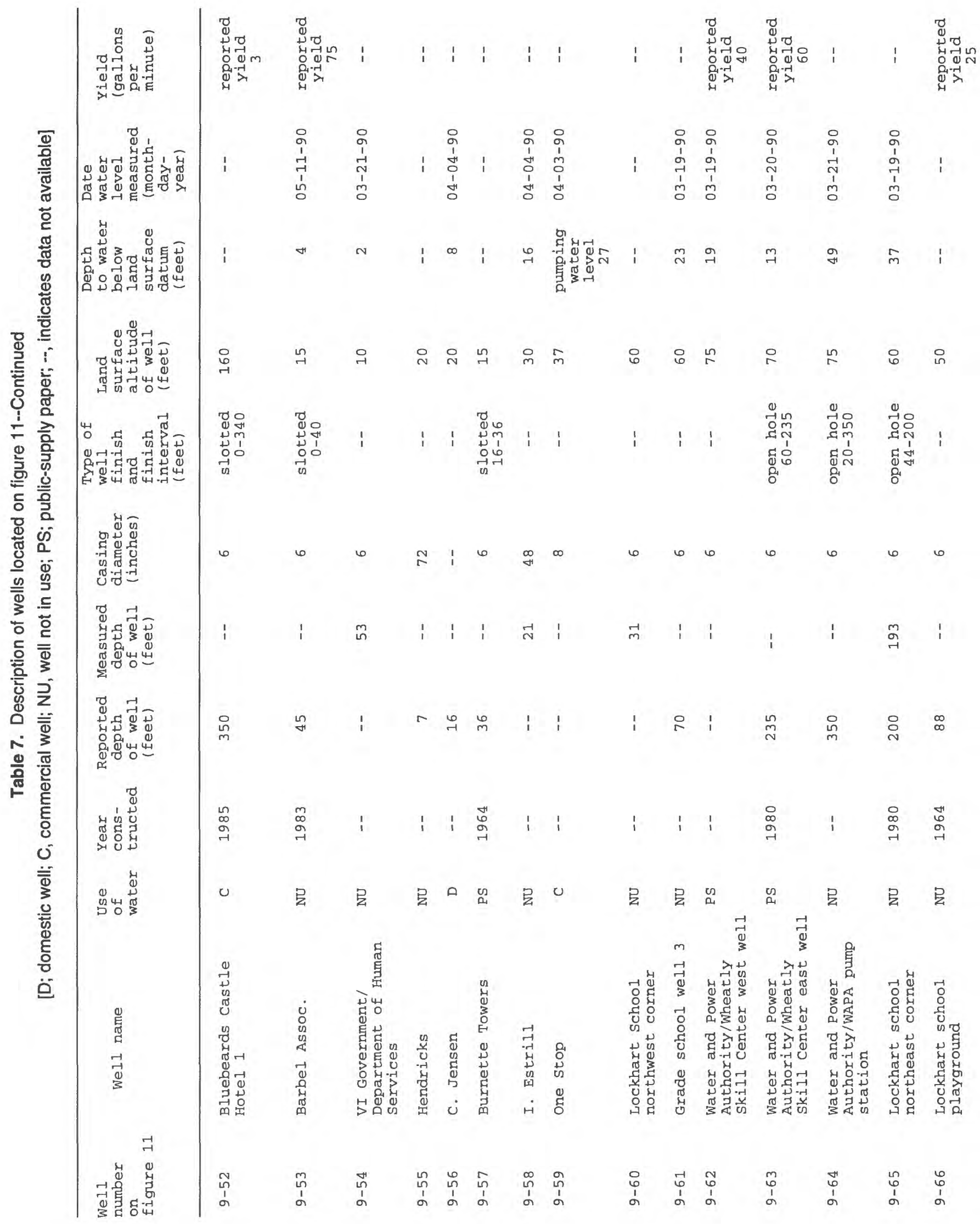




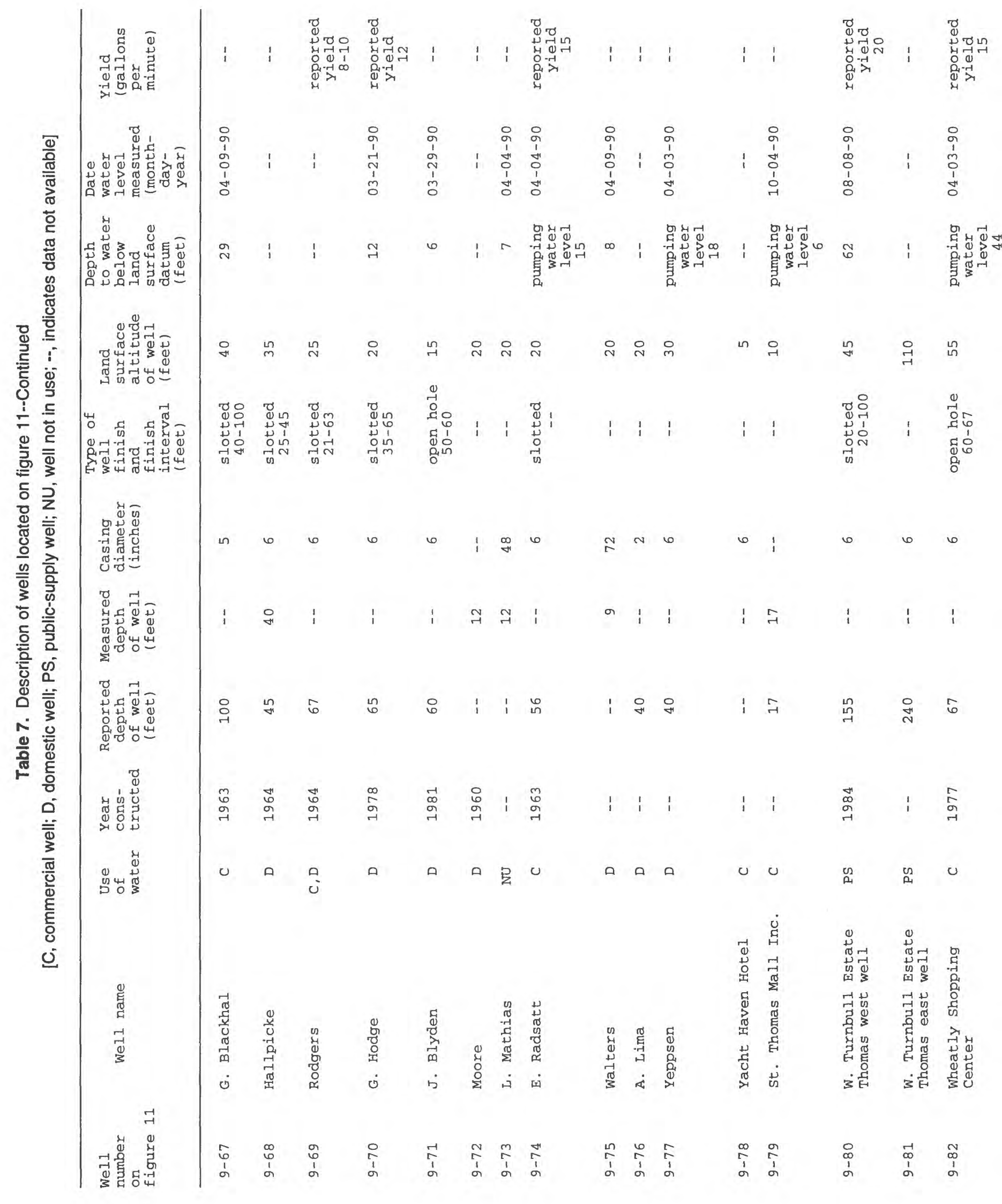




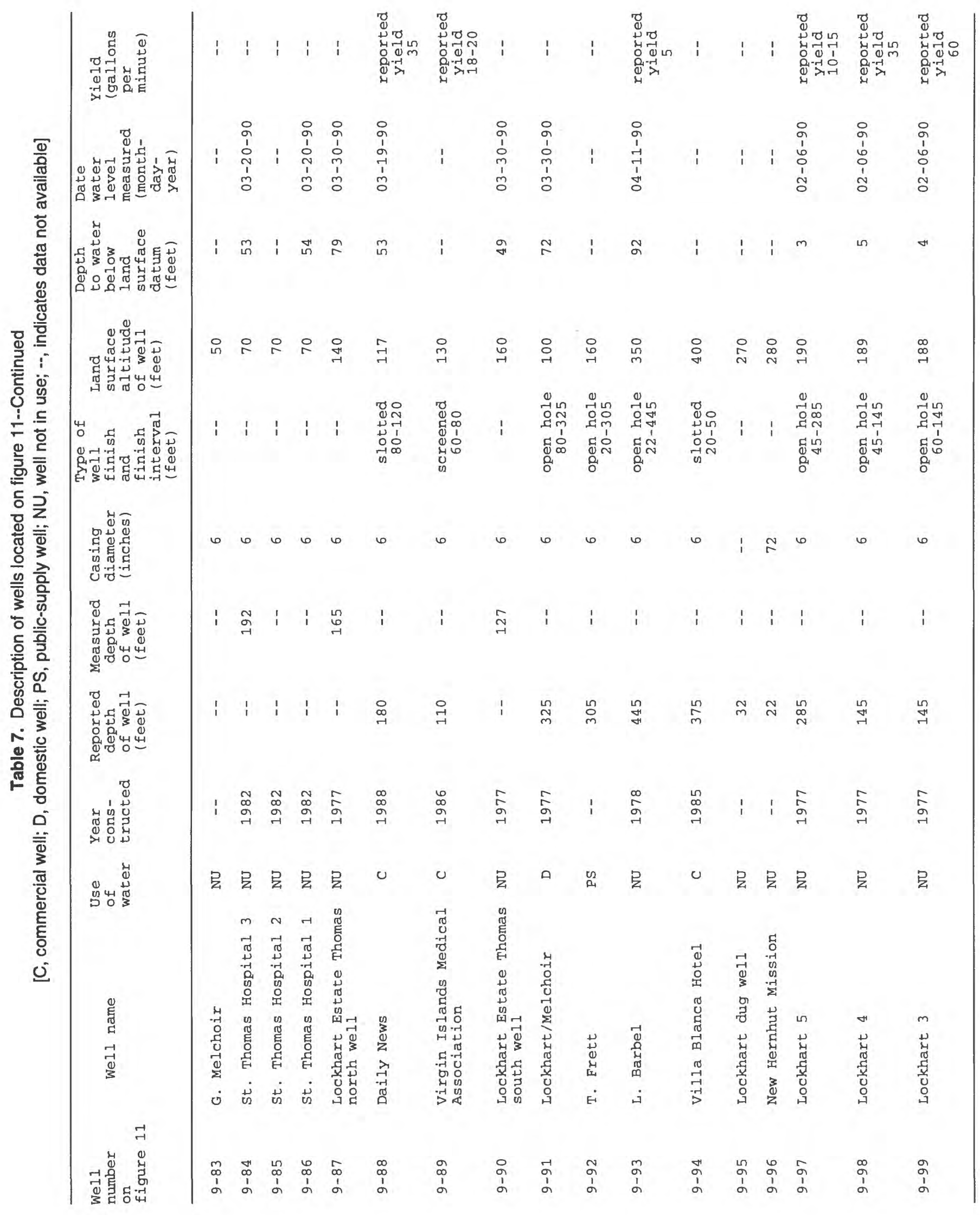




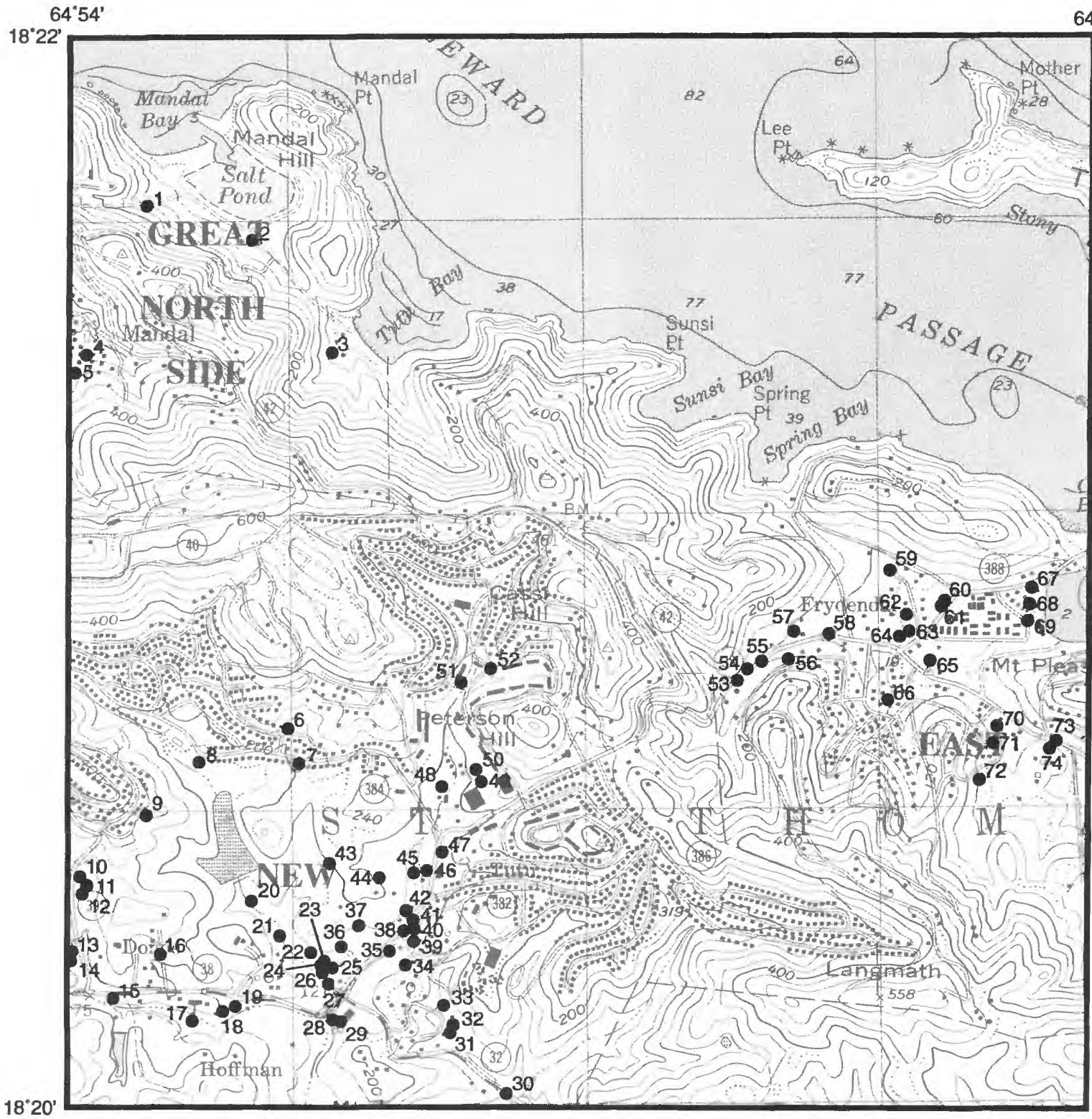

Base from U.S. Geological Survey

Eastern St. Thomas, V.I. 1:24,000, 1982 SCALE

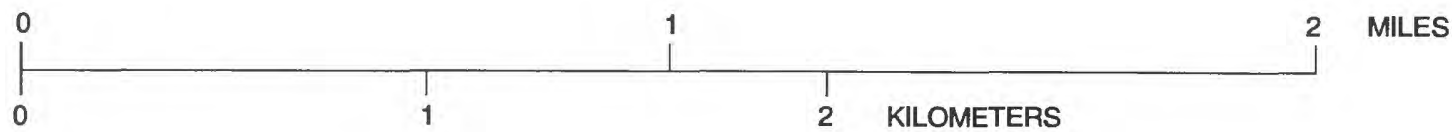

Figure 12. Location of wells on grid 10 of figure 1 . The well numbers shown on this figure correspond to the well numbers which begin with 10 - in table 8 and appendixes A and B. 


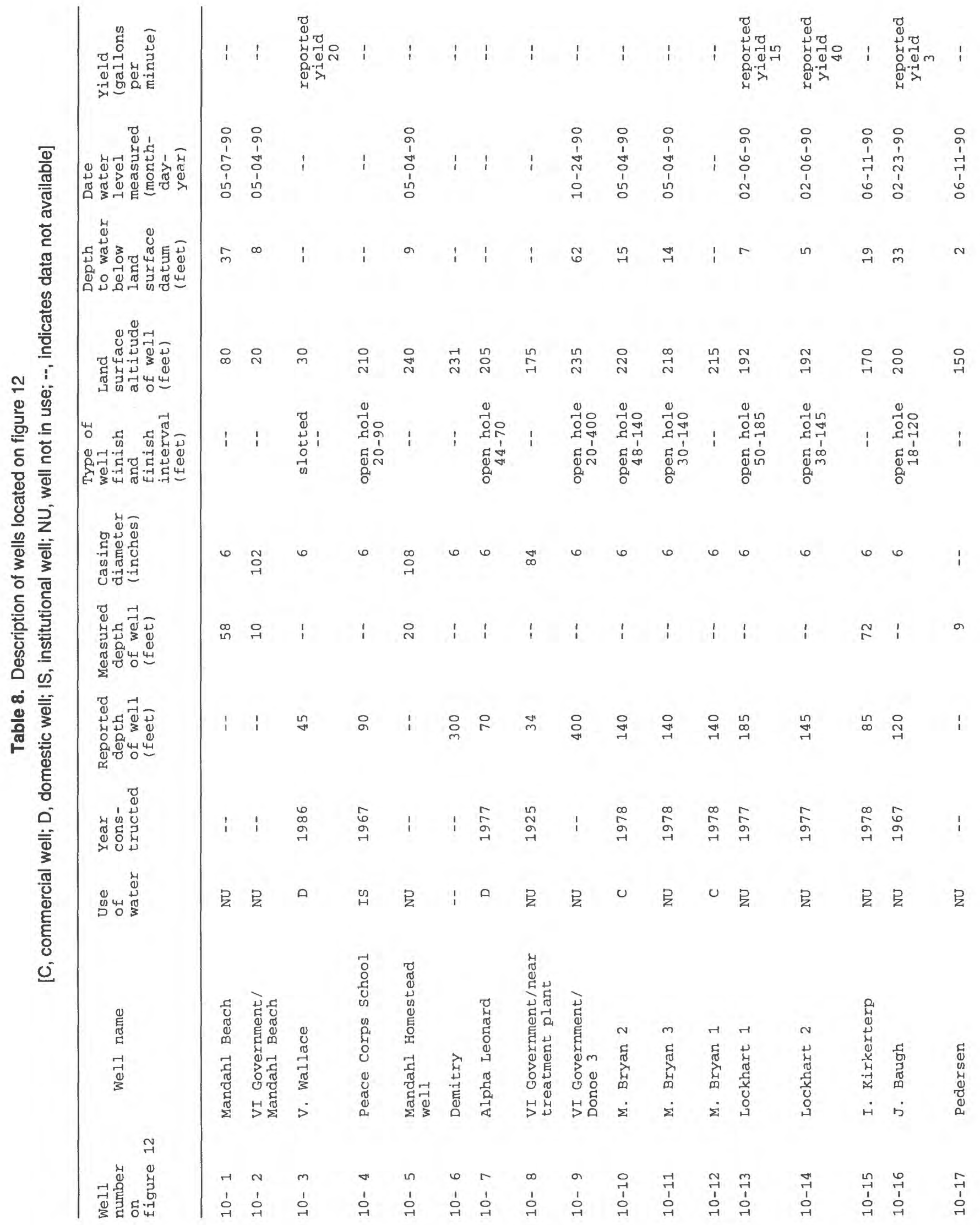




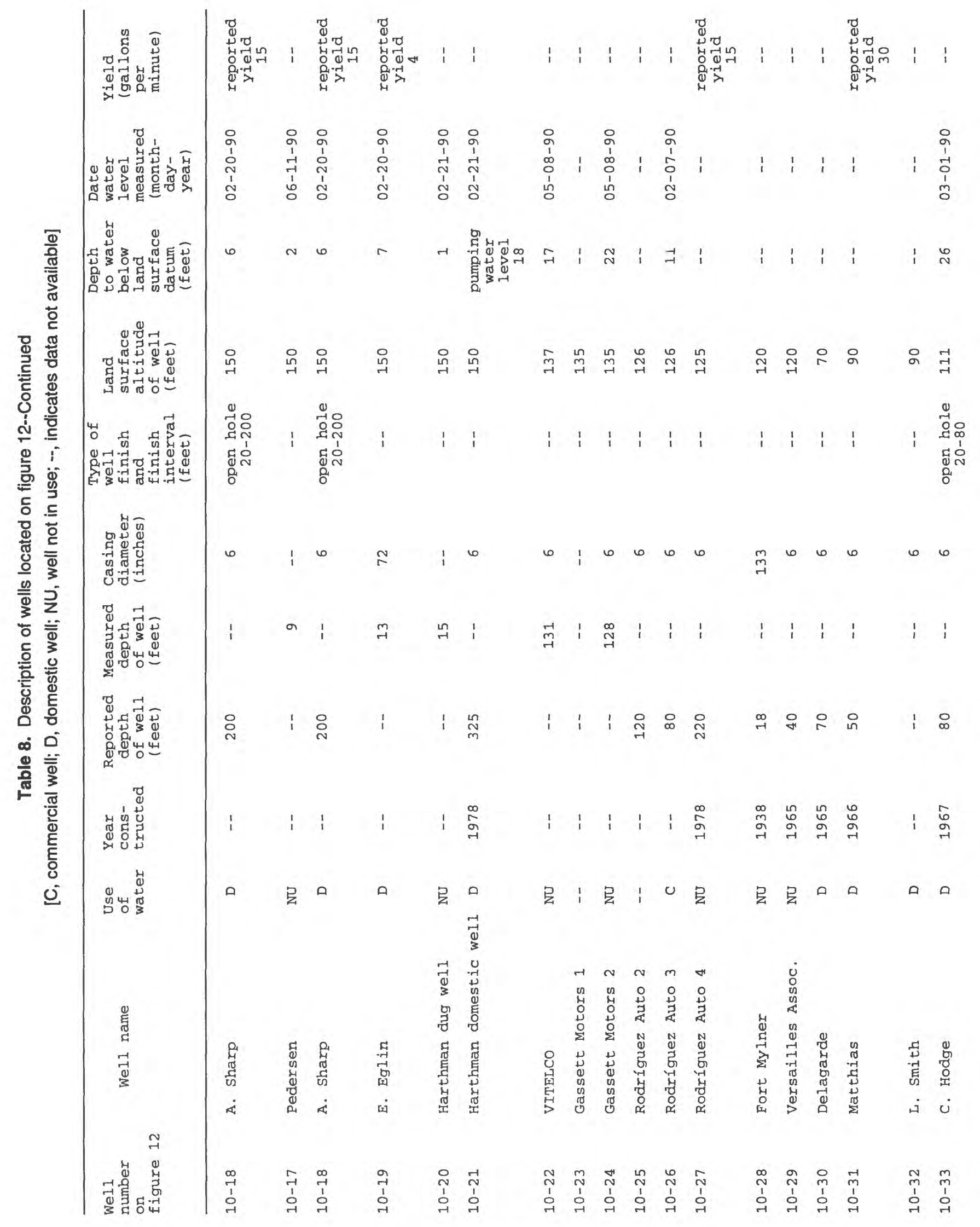




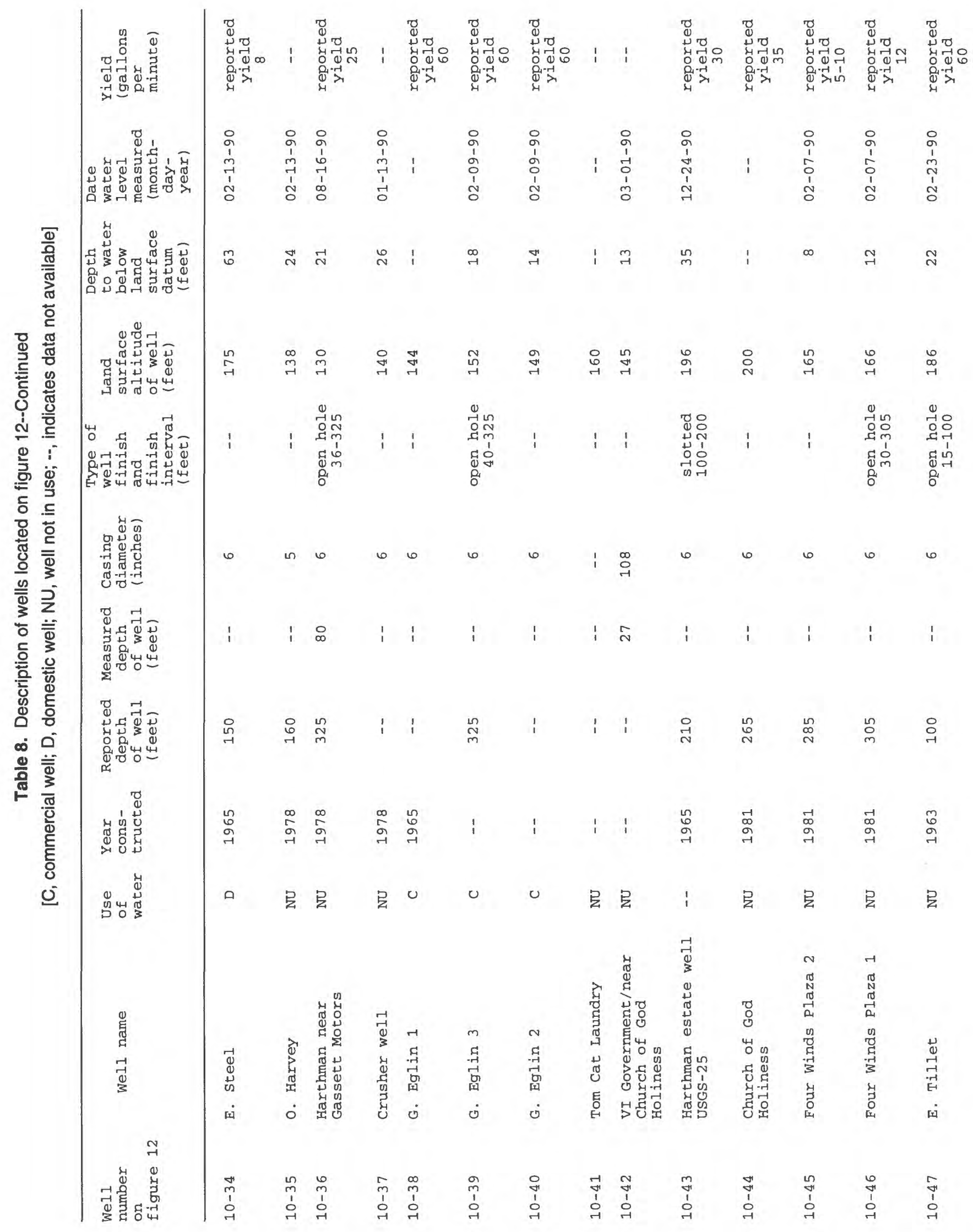




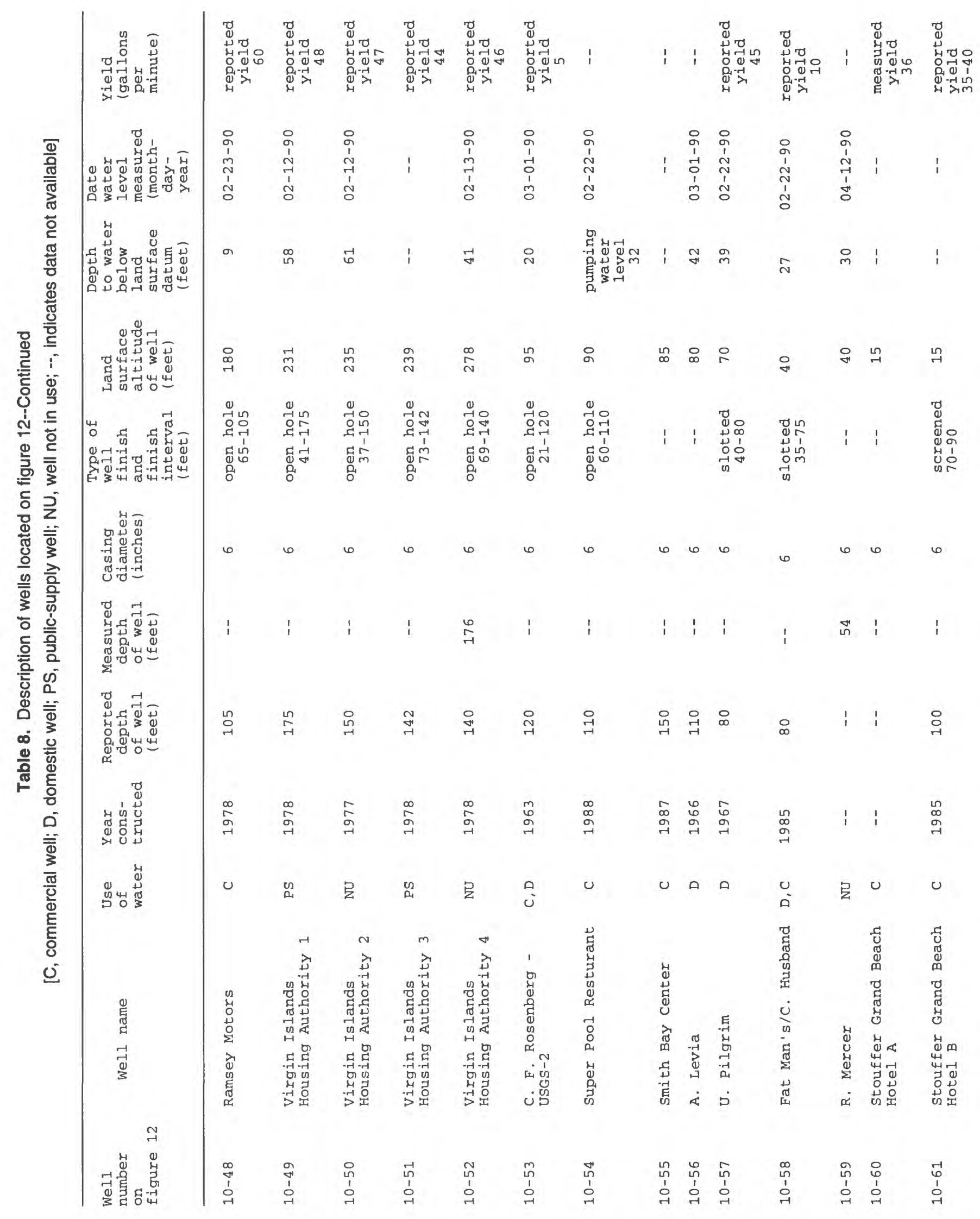




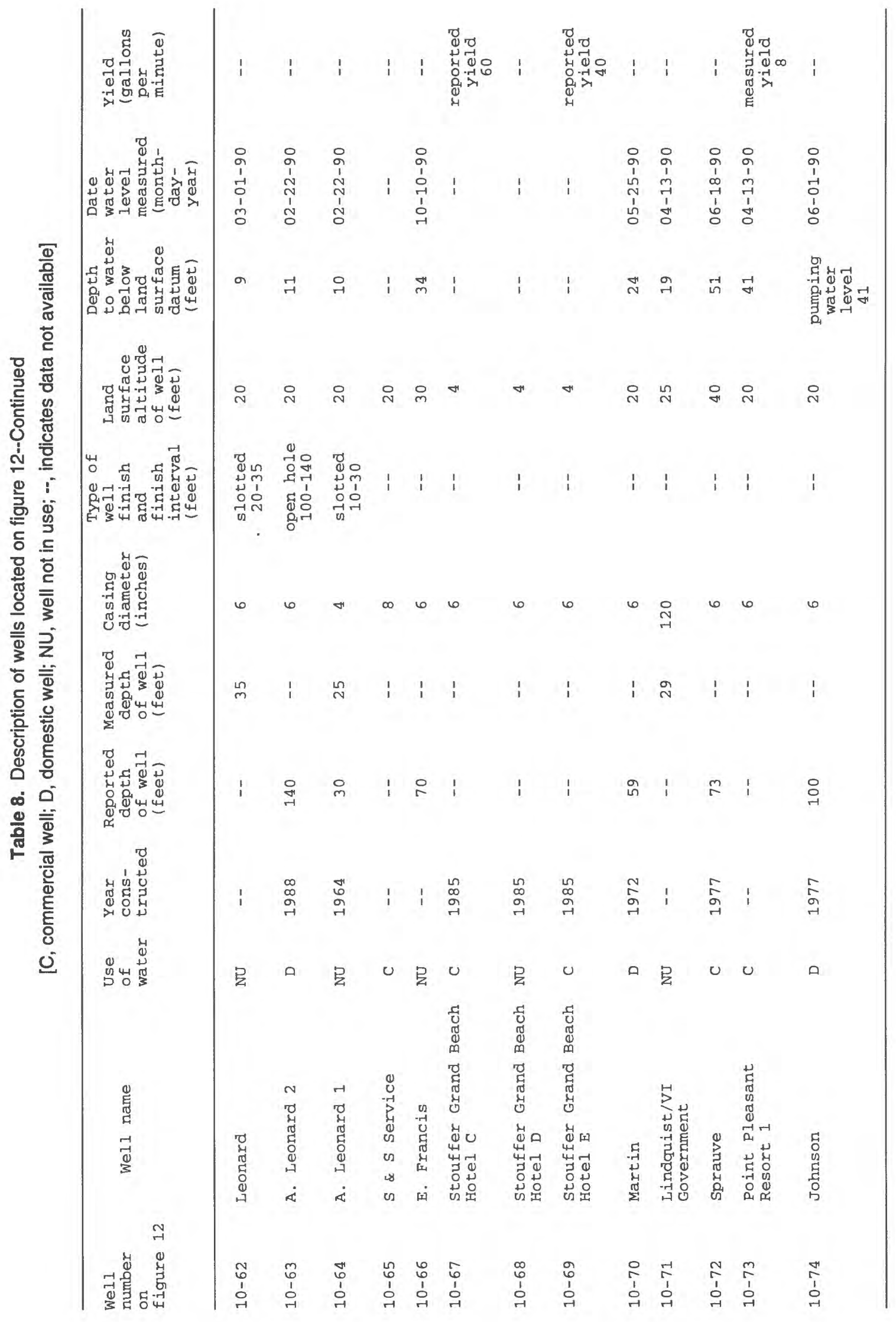




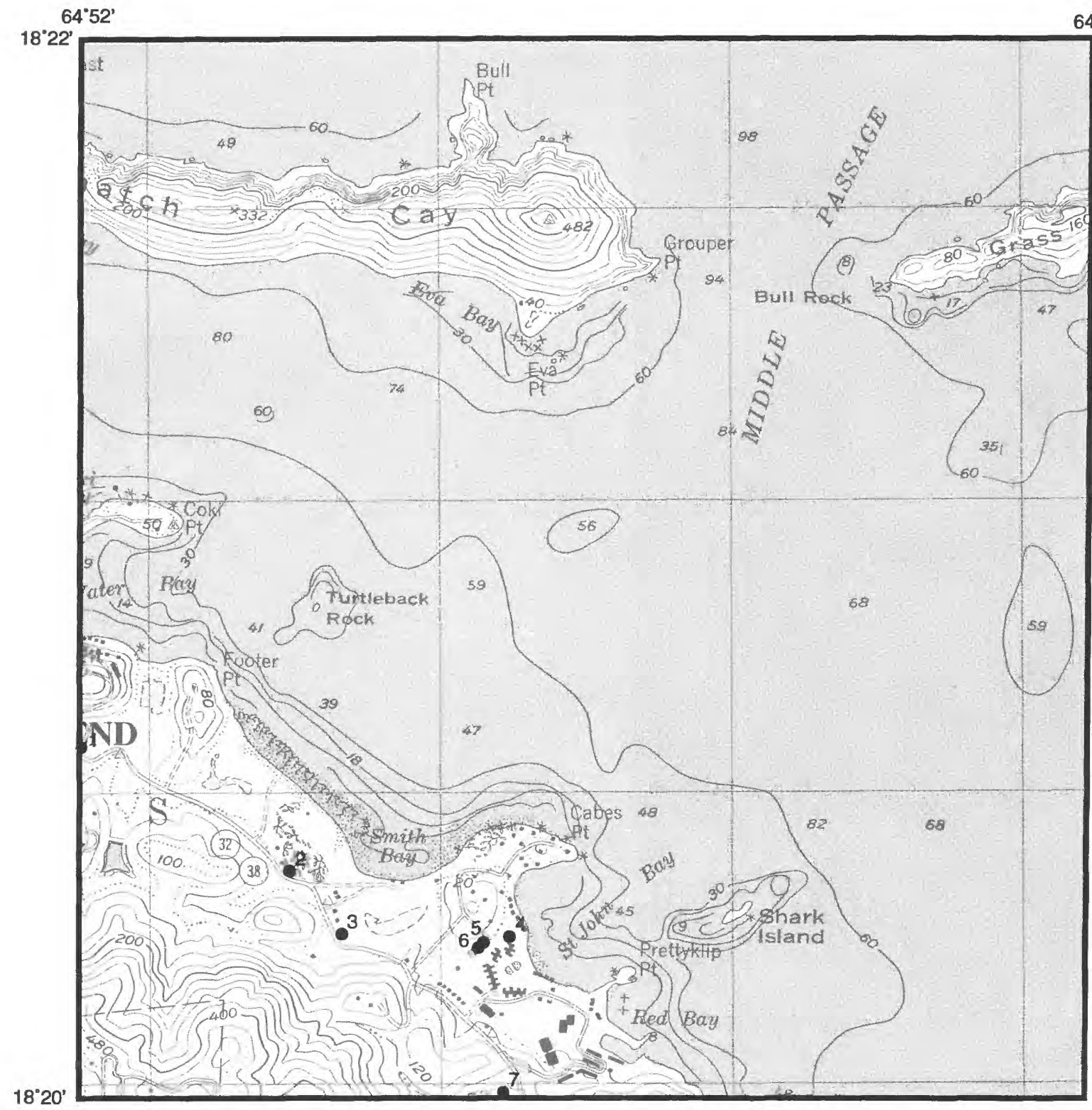

Base from U.S. Geological Survey

Eastern St. Thomas, V.I. 1:24,000, 1982 SCALE

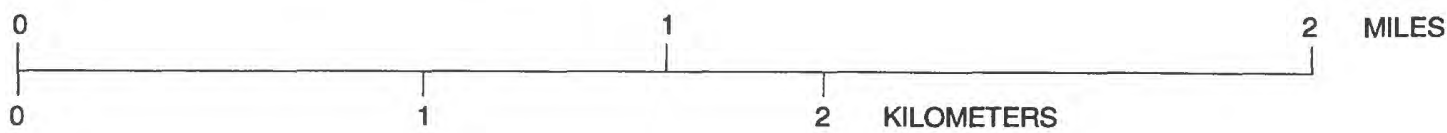

Figure 13. Location of wells on grid 11 of figure 1. The well numbers shown on this figure correspond to the well numbers which begin with 11 - in table 9 and appendixes A and B. 


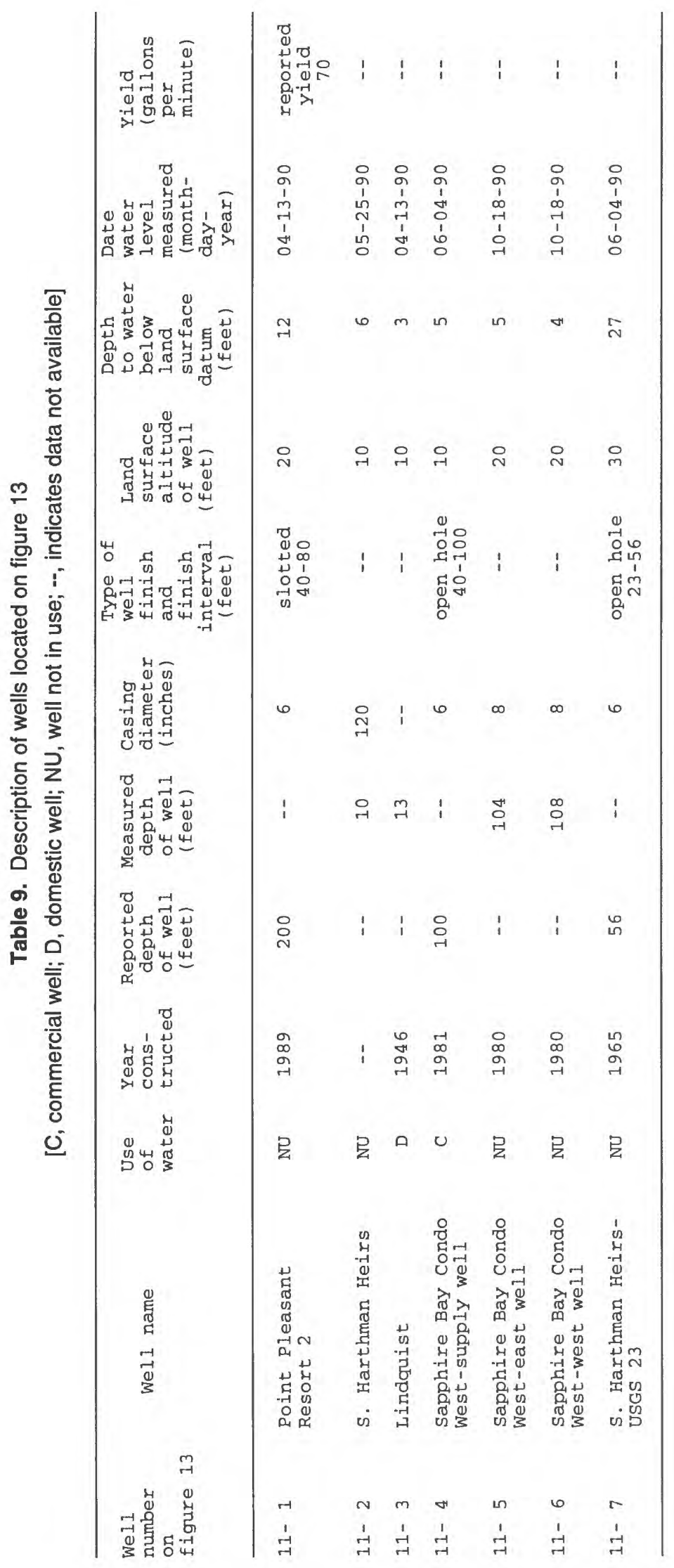



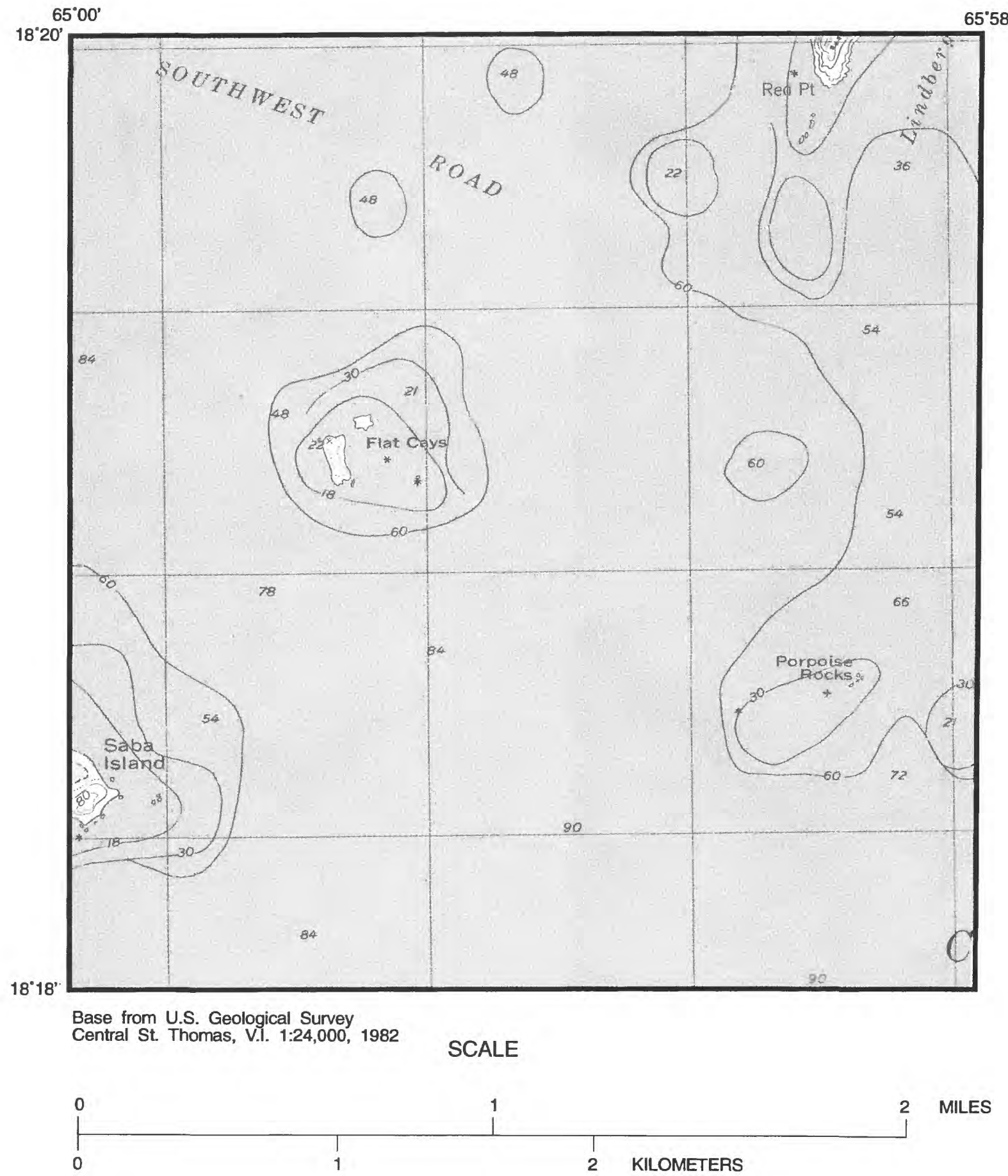

Figure 14. Grid 12 of figure 1. 


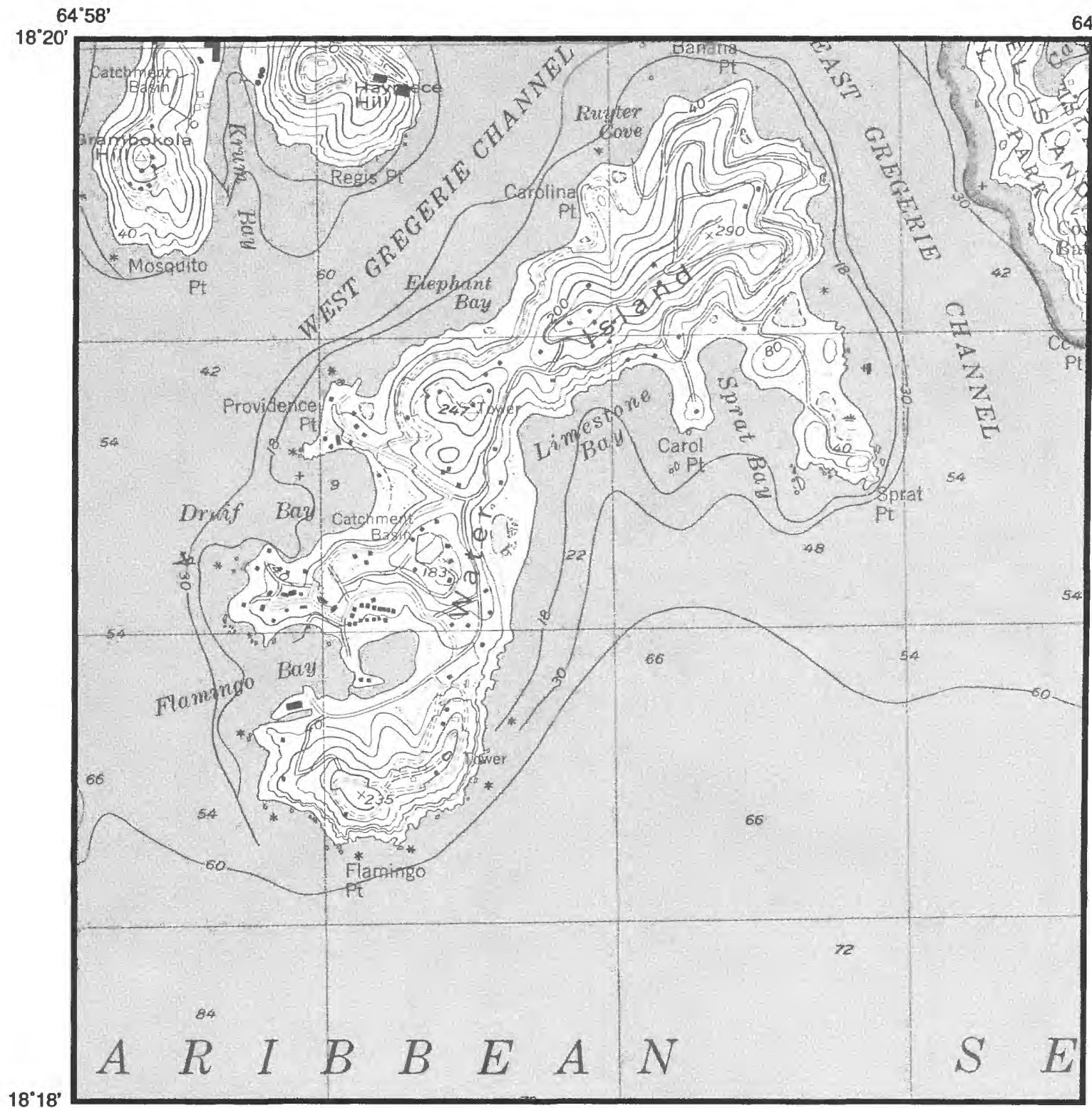

Base from U.S. Geological Survey

Central St. Thomas, V.I. 1:24,000, 1982

SCALE

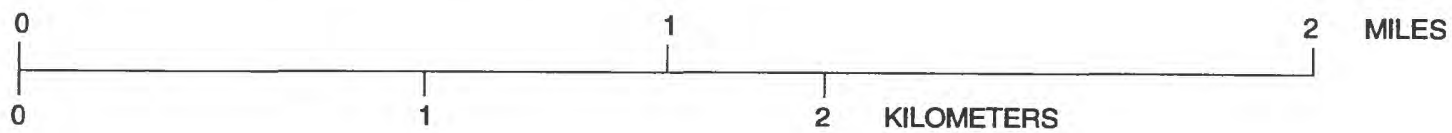

Figure 15. Grid 13 of figure 1. 


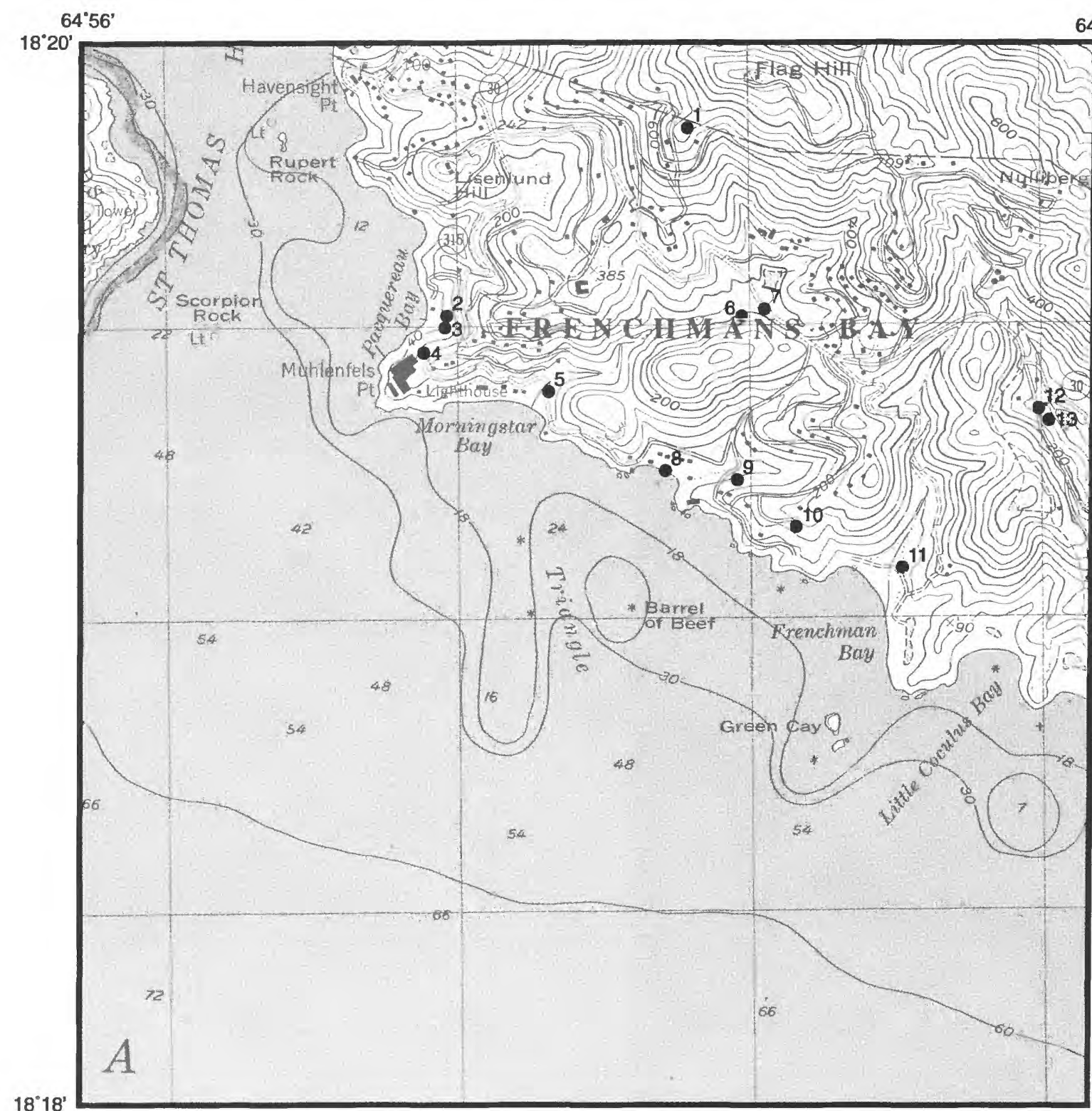

Base from U.S. Geological Survey

Central St. Thomas, V.1. 1:24,000, 1982 SCALE

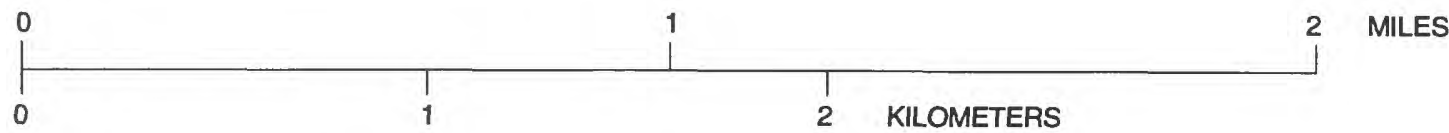

Figure 16. Location of wells on grid 14 of figure 1. The well numbers shown on this figure correspond to the well numbers which begin with 14 - in table 10 and appendixes $A$ and $B$. 


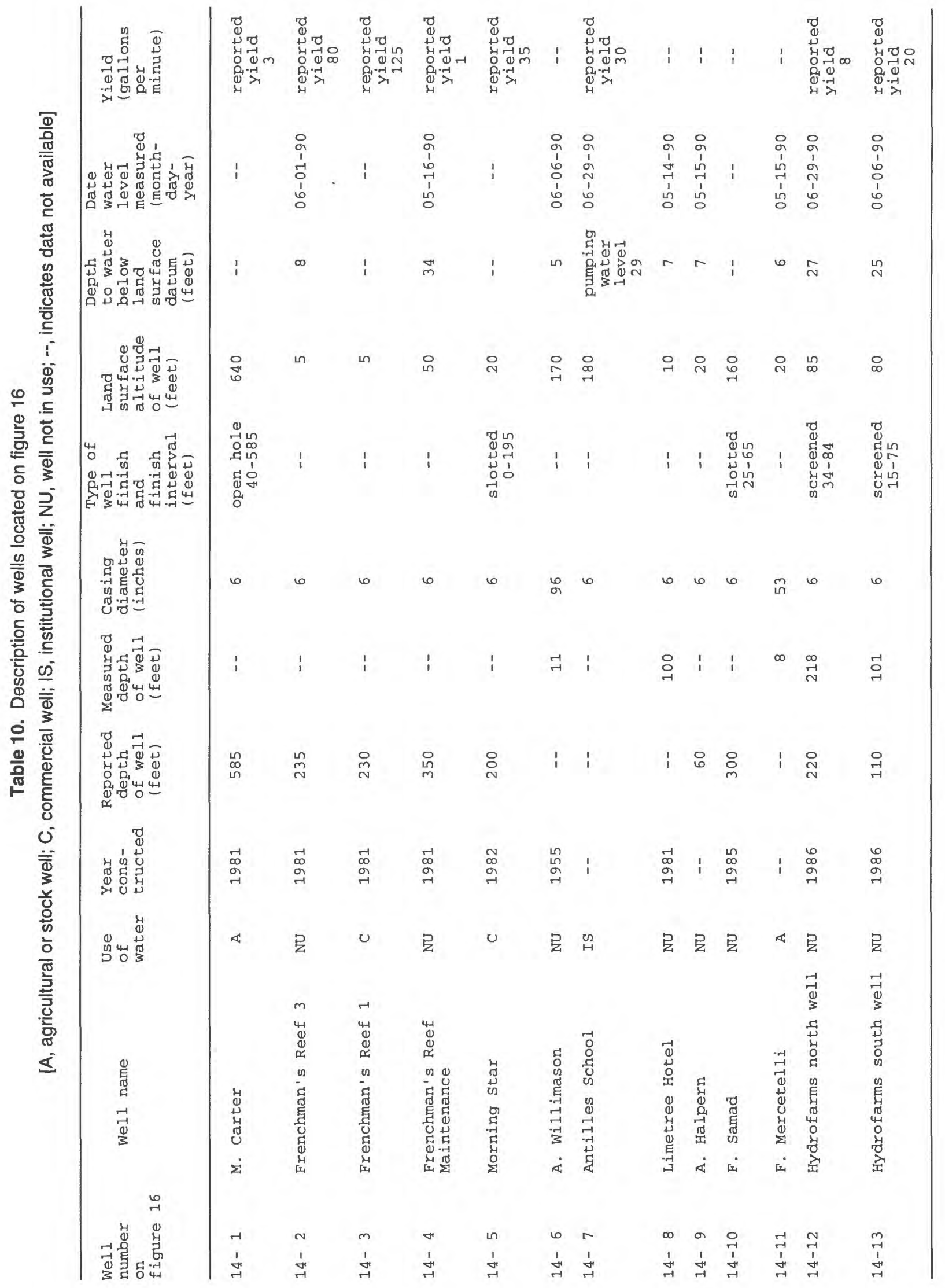




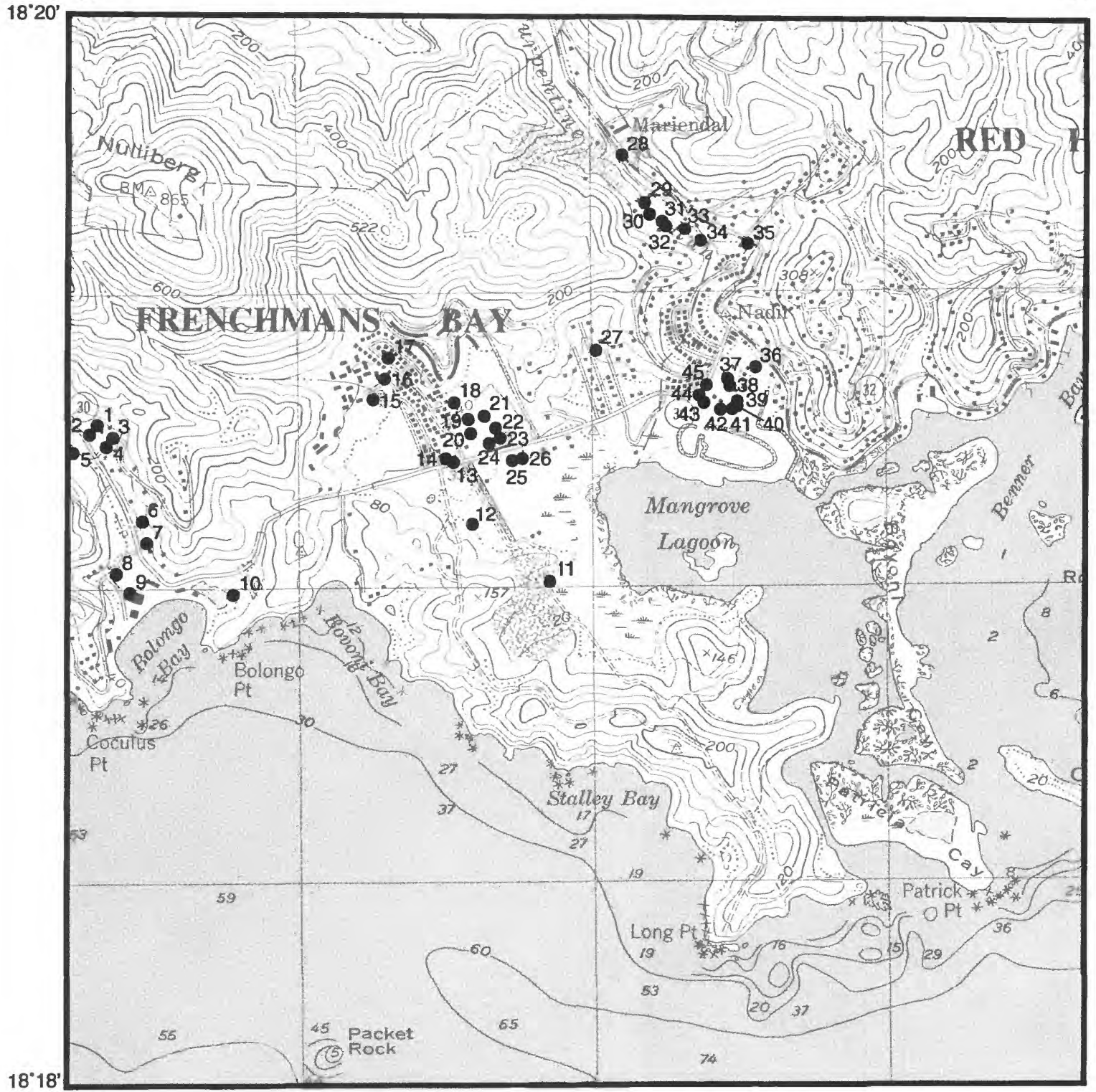

Base from U.S. Geological Survey Eastern St. Thomas, V.I. 1:24,000, 1982

SCALE

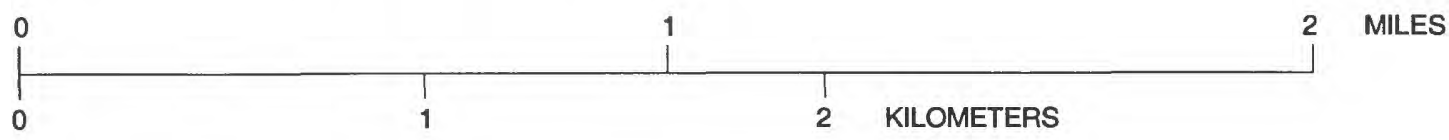

Figure 17. Location of wells on grid 15 of figure 1 . The well numbers shown on this figure correspond to the well numbers which begin with 15 - in table 11 and appendixes $A$ and $B$. 


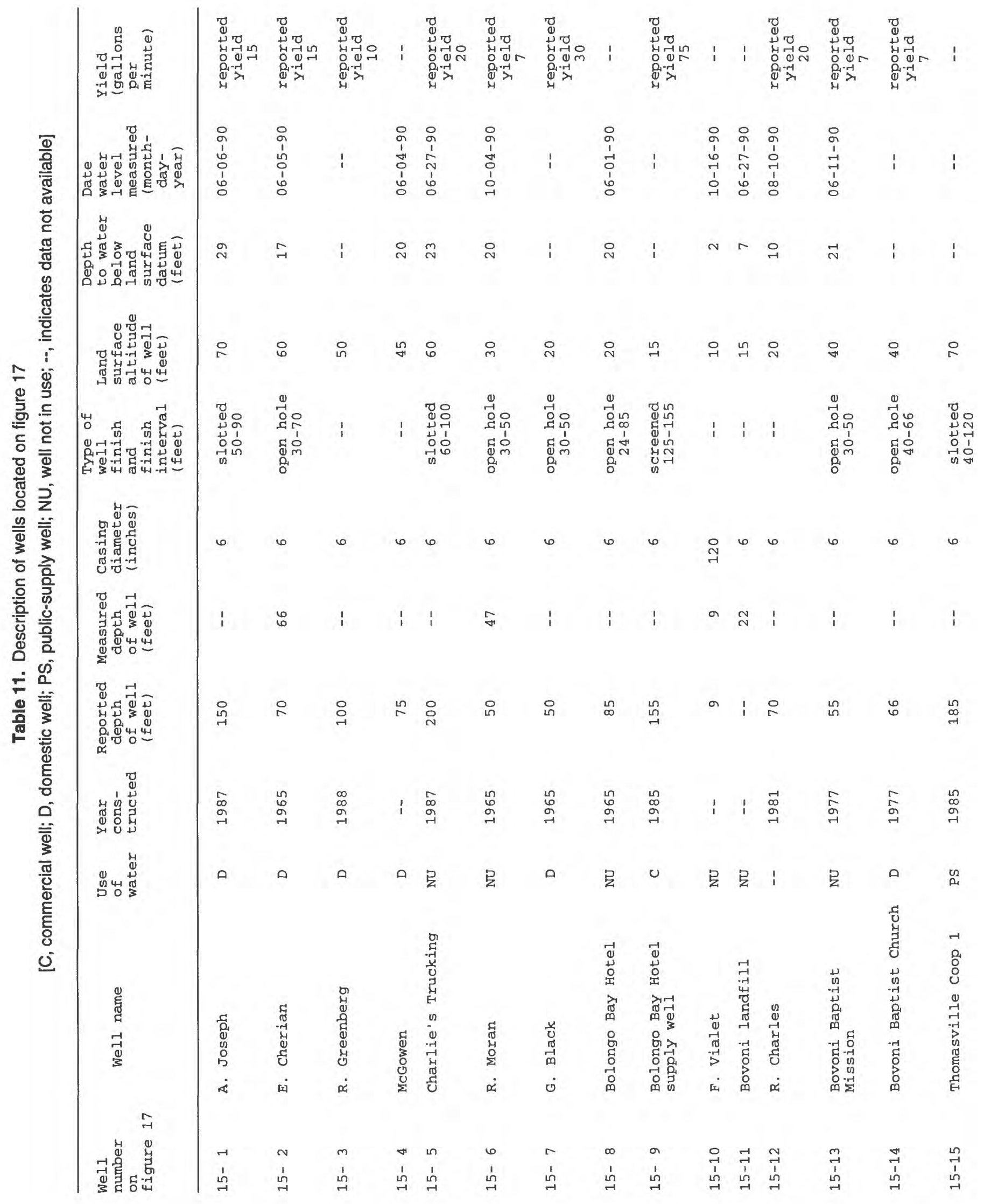




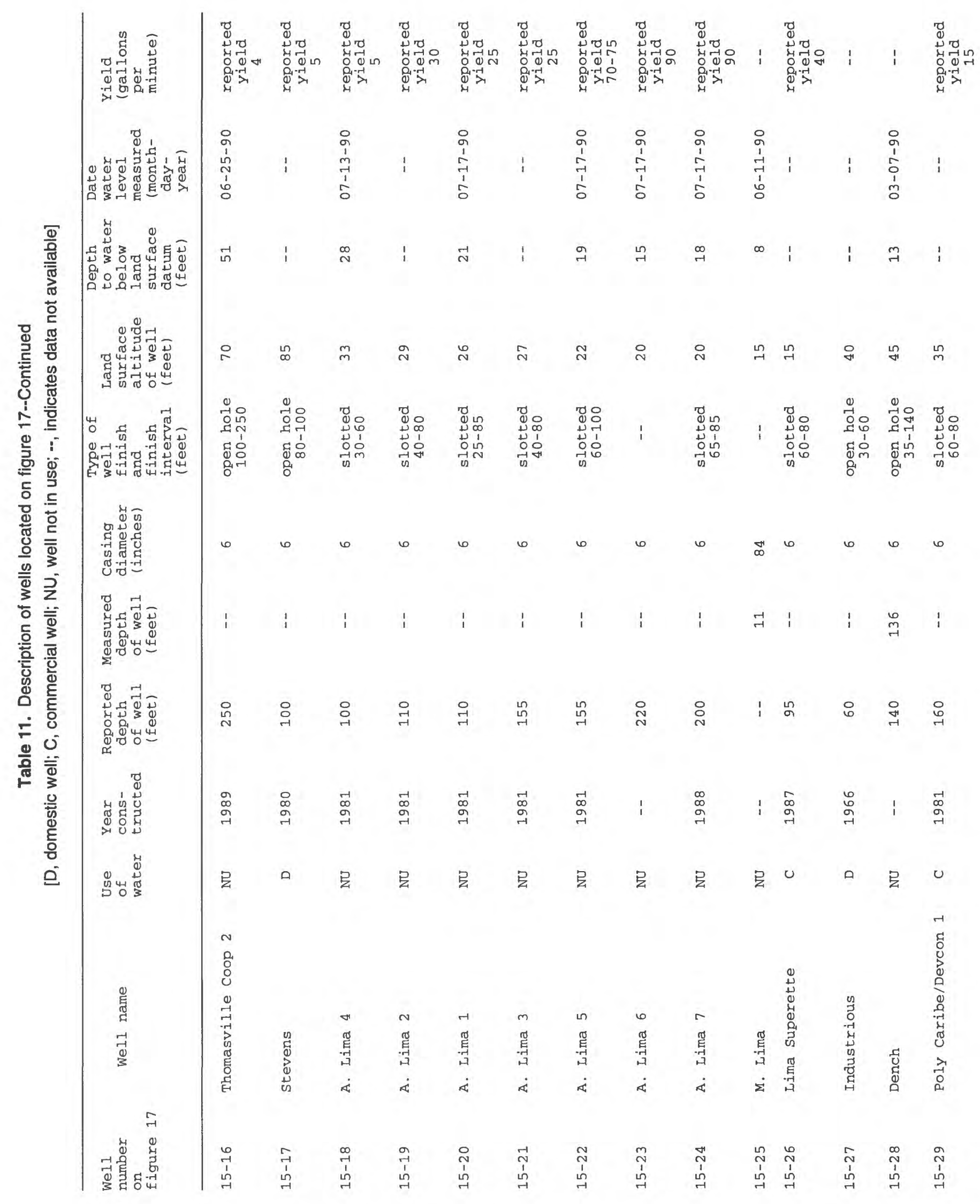




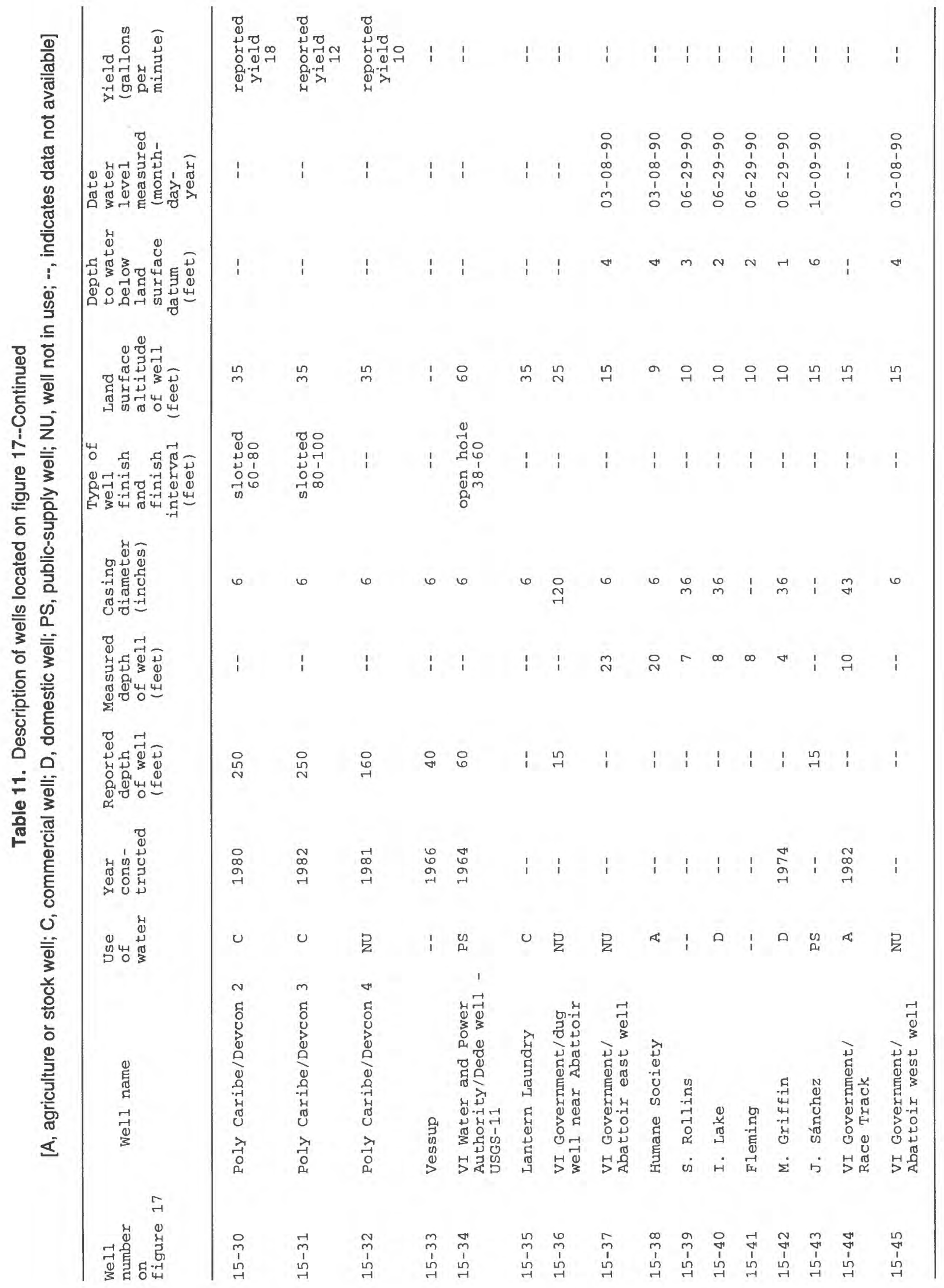




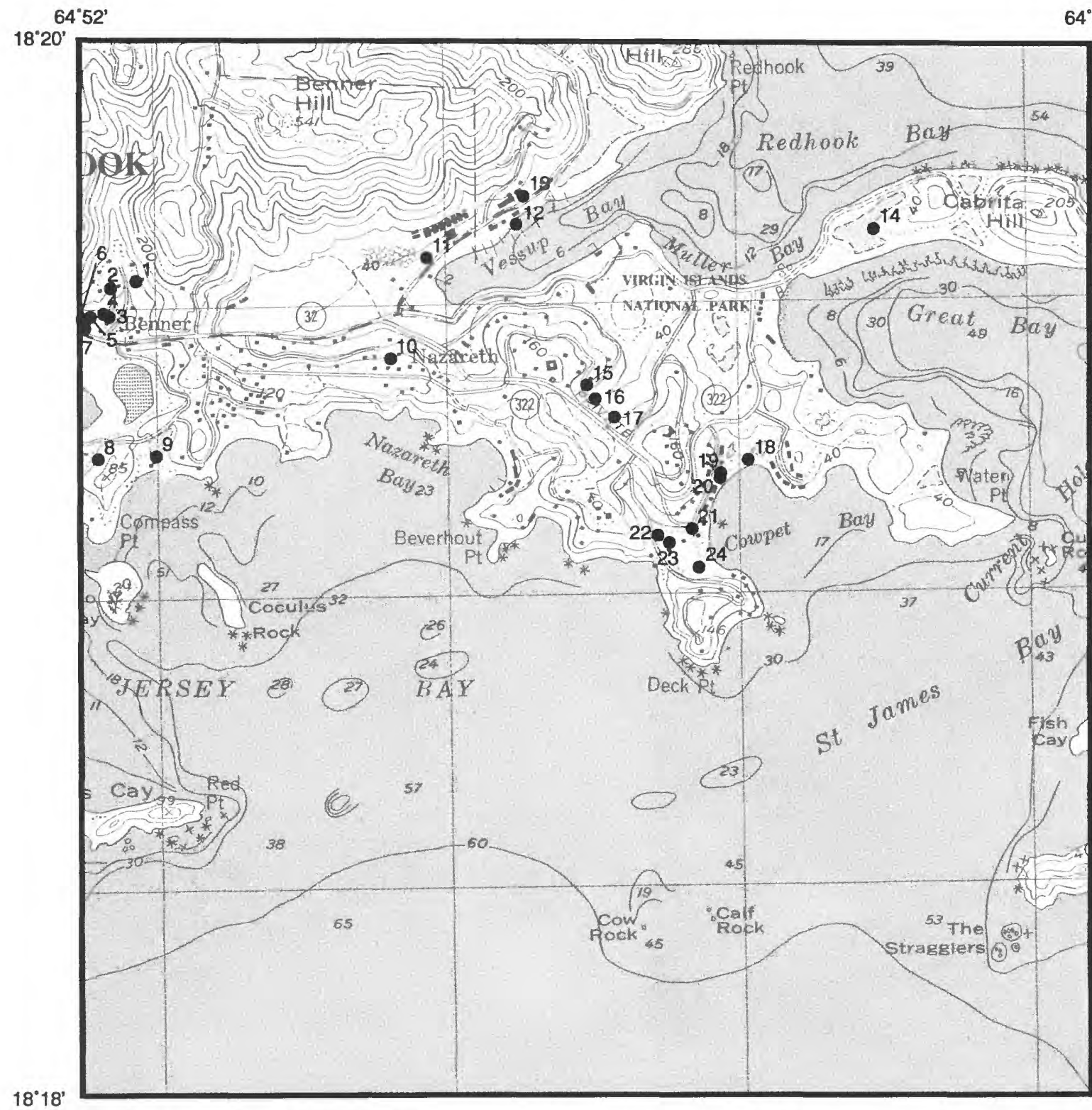

Base from U.S. Geological Survey

Eastern St. Thomas, V.I. 1:24,000, 1982 SCALE

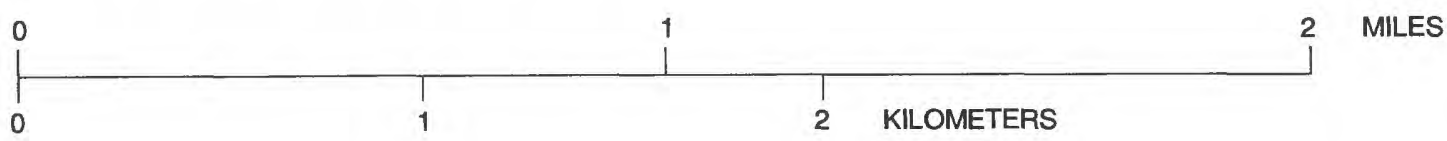

Figure 18. Location of wells on grid 16 of figure 1 . The well numbers shown on this figure correspond to the well numbers which begin with 16- in table 12 and appendixes $A$ and $B$. 


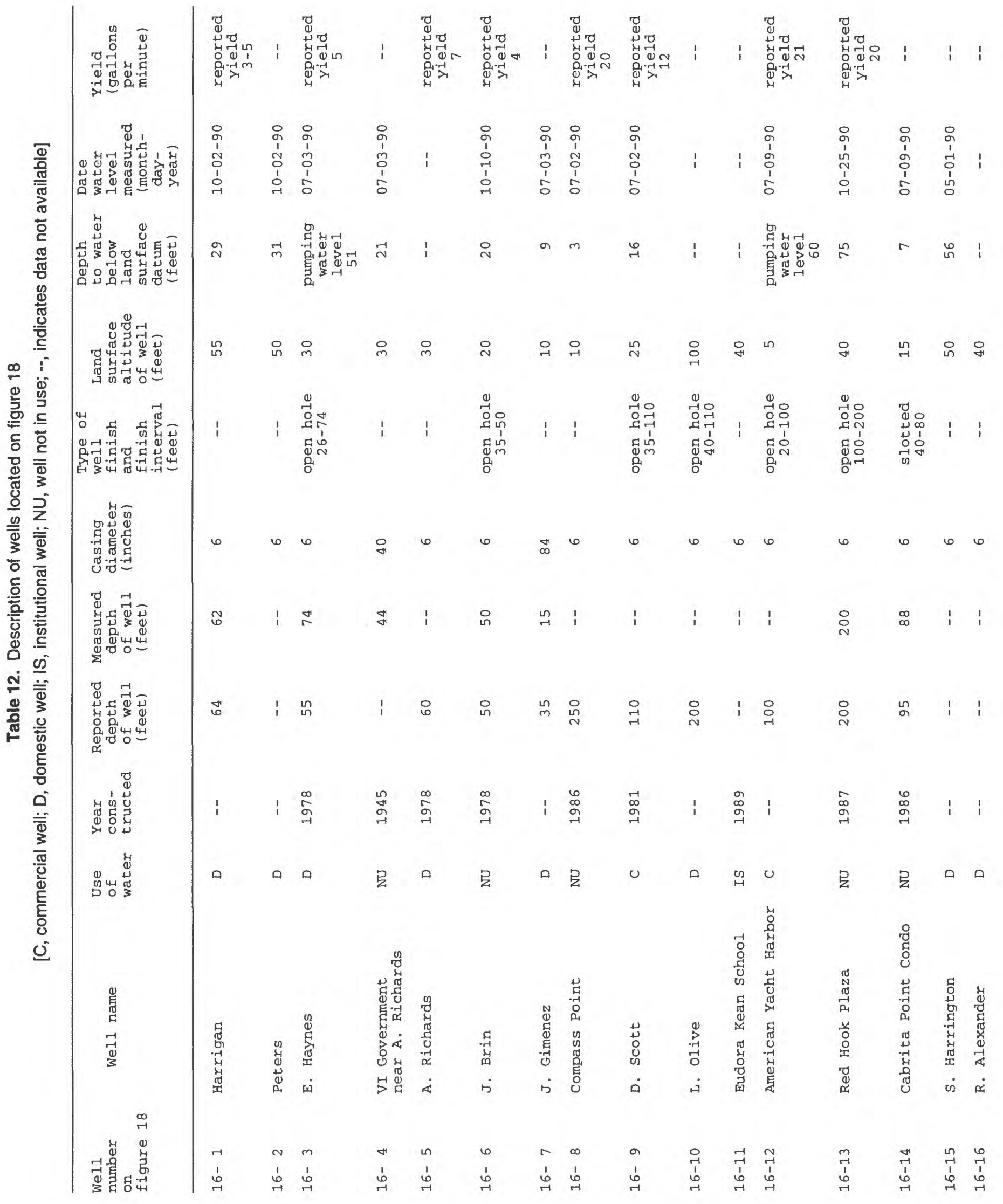




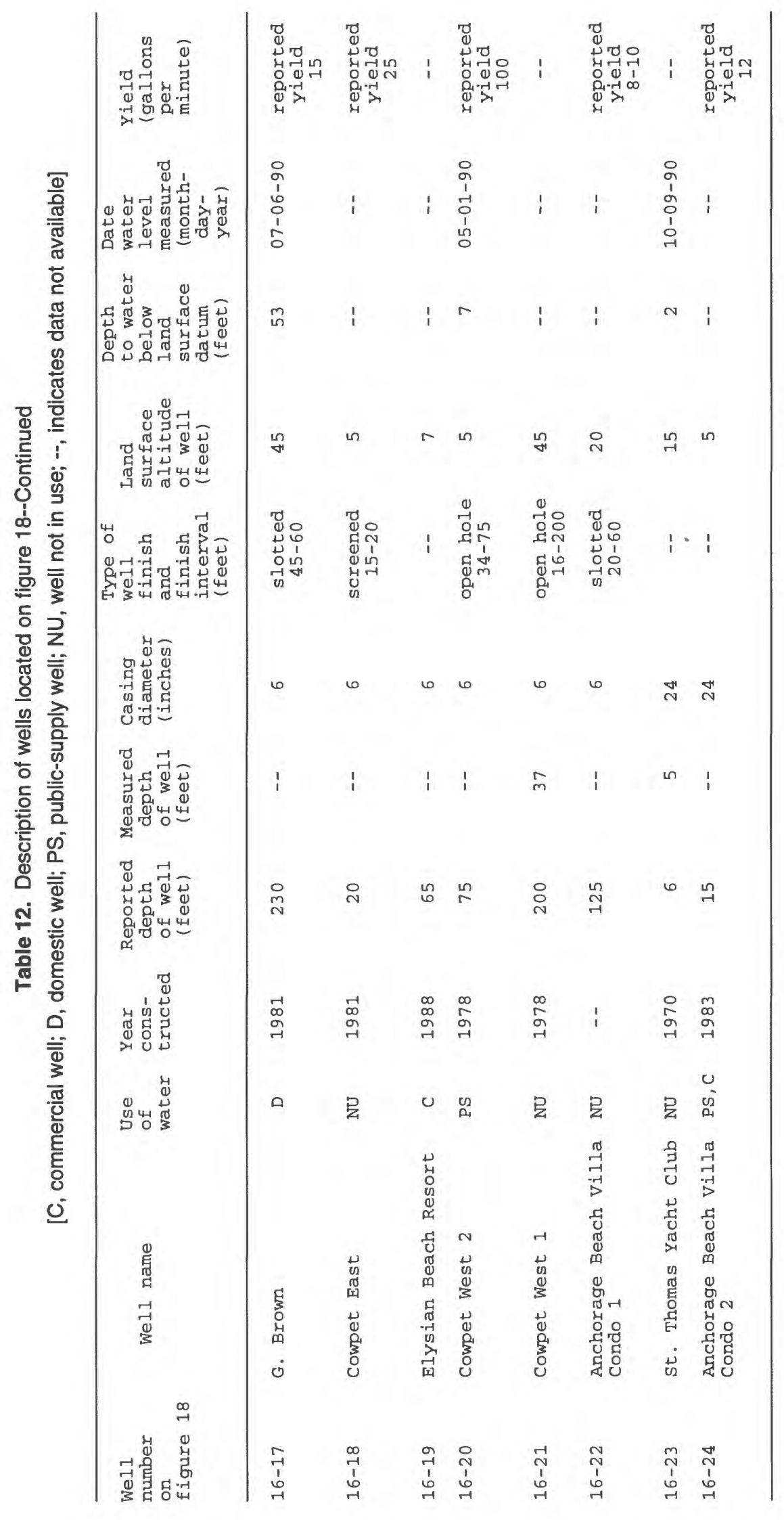



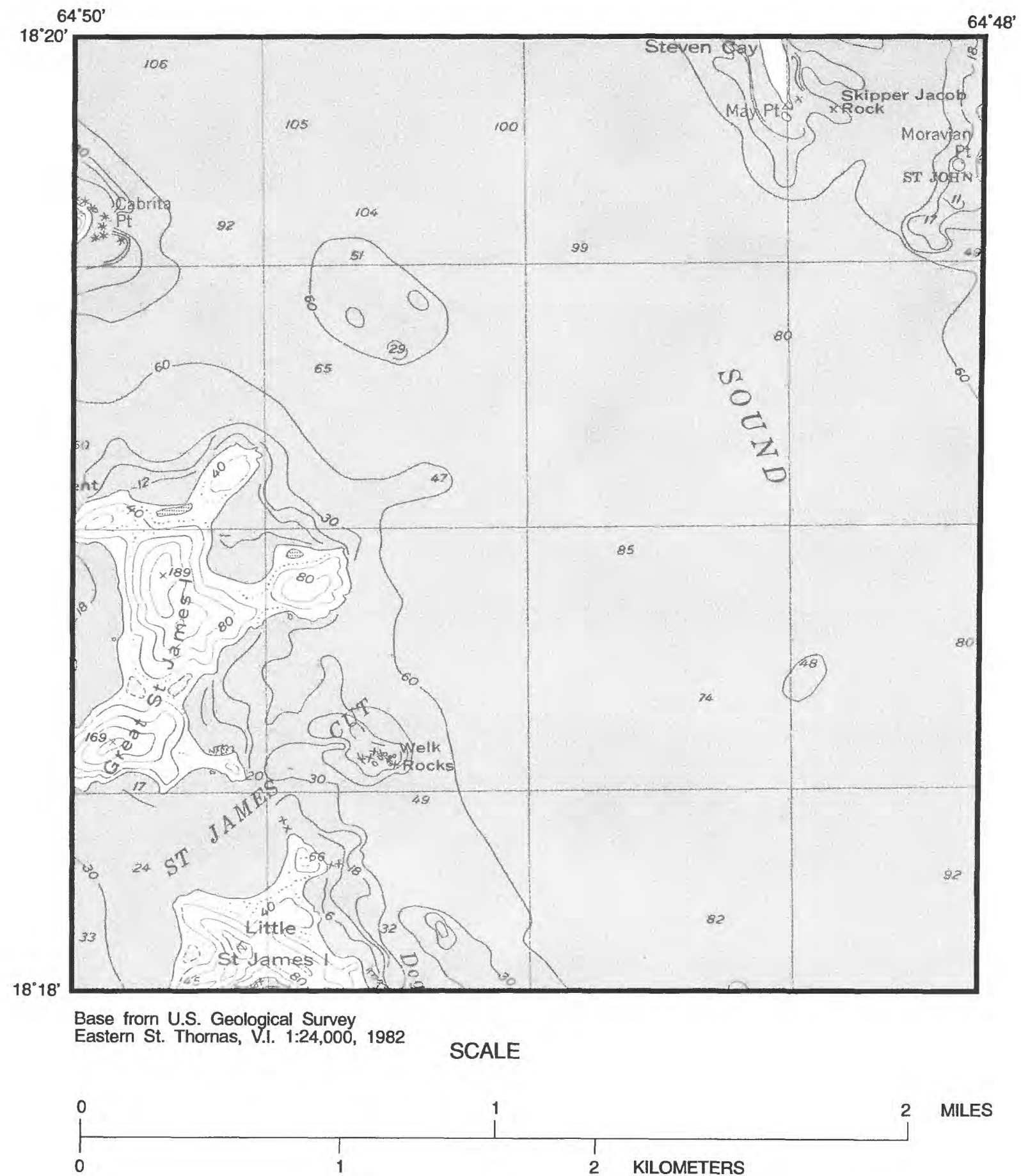

Figure 19. Grid 17 of figure 1. 
Appendix A. Well names, Estates, and site-identification numbers of wells on St. Thomas, U.S. Virgin Islands

\begin{tabular}{|c|c|c|c|c|}
\hline $\begin{array}{l}\text { We11 } \\
\text { number }\end{array}$ & $\begin{array}{l}\text { Figure } \\
\text { number }\end{array}$ & Well name & $\begin{array}{c}\text { Estate } \\
\text { (see figure 2) }\end{array}$ & $\begin{array}{c}\text { Site } \\
\text { identification } \\
\text { number }\end{array}$ \\
\hline $2-1$ & 4 & C. Bushfield & Caret Bay & 182214064584500 \\
\hline $3-1$ & 5 & $\begin{array}{l}\text { Dorothea Beach } \\
\text { Resort } 2\end{array}$ & Dorothea & 182213064574500 \\
\hline $3-2$ & 5 & $\begin{array}{l}\text { Dorothea Beach } \\
\text { Resort } 3\end{array}$ & Dorothea & 182212064574400 \\
\hline $3-3$ & 5 & $\begin{array}{l}\text { Dorothea Beach } \\
\text { Resort } 4\end{array}$ & Dorothea & 182212064574401 \\
\hline $3-4$ & 5 & $\begin{array}{l}\text { Dorothea Beach } \\
\text { Resort dug well }\end{array}$ & Dorothea & 182214064574200 \\
\hline $3-5$ & 5 & $\begin{array}{l}\text { Dorothea Beach } \\
\text { Resort } 8\end{array}$ & Dorothea & 182213064574101 \\
\hline $3-6$ & 5 & $\begin{array}{l}\text { Dorothea Beach } \\
\text { Resort } 7\end{array}$ & Dorothea & 182213064574100 \\
\hline $3-7$ & 5 & $\begin{array}{l}\text { Dorothea Beach } \\
\text { Resort } 6\end{array}$ & Dorothea & 182213064574200 \\
\hline $3-8$ & 5 & $\begin{array}{l}\text { Dorothea Beach } \\
\text { Resort } 5\end{array}$ & Dorothea & 182212064574200 \\
\hline $3-9$ & 5 & $\begin{array}{l}\text { Dorothea Beach } \\
\text { Resort } 9\end{array}$ & Dorothea & 182212064574201 \\
\hline $3-10$ & 5 & $\begin{array}{l}\text { Dorothea Beach } \\
\text { Resort Gut well }\end{array}$ & Dorothea & 182212064573900 \\
\hline $3-11$ & 5 & R. Laplace & Hull & 182214064571700 \\
\hline $3-12$ & 5 & Hull Hideaway & Hull & 182215064570800 \\
\hline $3-13$ & 5 & L. Laplace & Hul1 & 182215064570700 \\
\hline $3-14$ & 5 & $\begin{array}{l}\text { L. Laplace } \\
\text { irrigation well }\end{array}$ & Hull & 182215064570500 \\
\hline $3-15$ & 5 & L. \& S. Bryan & Hul1 & 182215064565800 \\
\hline $3-16$ & 5 & A. Newmann & st. Peter & 182216064564000 \\
\hline $6-1$ & 8 & W. Wernicke & Fortuna & 182046065010500 \\
\hline $7-1$ & 9 & $\begin{array}{l}\text { Perseverance Bay } \\
\text { well }\end{array}$ & Perseverance & 182116064595400 \\
\hline $7-2$ & 9 & B. Nelthrup & Lindberg Bay & 182129064581200 \\
\hline $7-3$ & 9 & $\begin{array}{l}\text { University of the } \\
\text { Virgin Islands } \\
\text { Reichold Center D }\end{array}$ & $\begin{array}{l}\text { John Brewer's } \\
\text { Bay }\end{array}$ & 182050064583100 \\
\hline $7-4$ & 9 & $\begin{array}{l}\text { University of the } \\
\text { Virgin Islands } \\
\text { Reichold Center A }\end{array}$ & $\begin{array}{l}\text { John Brewer's } \\
\text { Bay }\end{array}$ & 182047064583100 \\
\hline $7-5$ & 9 & $\begin{array}{l}\text { University of the } \\
\text { Virgin Islands } \\
\text { Reichold center C }\end{array}$ & $\begin{array}{l}\text { John Brewer's } \\
\text { Bay }\end{array}$ & 182047064583000 \\
\hline $7-6$ & 9 & $\begin{array}{l}\text { University of the } \\
\text { Virgin Islands } \\
\text { Reichold Center B }\end{array}$ & $\begin{array}{l}\text { John Brewer's } \\
\text { Bay }\end{array}$ & 182048064582900 \\
\hline $7-7$ & 9 & $\begin{array}{l}\text { University of the } \\
\text { Virgin Islands } 3\end{array}$ & Lindberg Bay & 182045064581700 \\
\hline $7-8$ & 9 & $\begin{array}{l}\text { University of the } \\
\text { Virgin Islands } 2\end{array}$ & Lindberg Bay & 182039064581500 \\
\hline $7-9$ & 9 & $\begin{array}{l}\text { University of the } \\
\text { Virgin Islands } 1\end{array}$ & Lindberg Bay & 182036064581300 \\
\hline $7-10$ & 9 & $\begin{array}{l}\text { University of the } \\
\text { Virgin Islands } s 1\end{array}$ & Lindberg Bay & 182033064581400 \\
\hline
\end{tabular}


Appendix A. Well names, Estates, and site-identification numbers of wells on St. Thomas, U.S. Virgin Islands--Continued

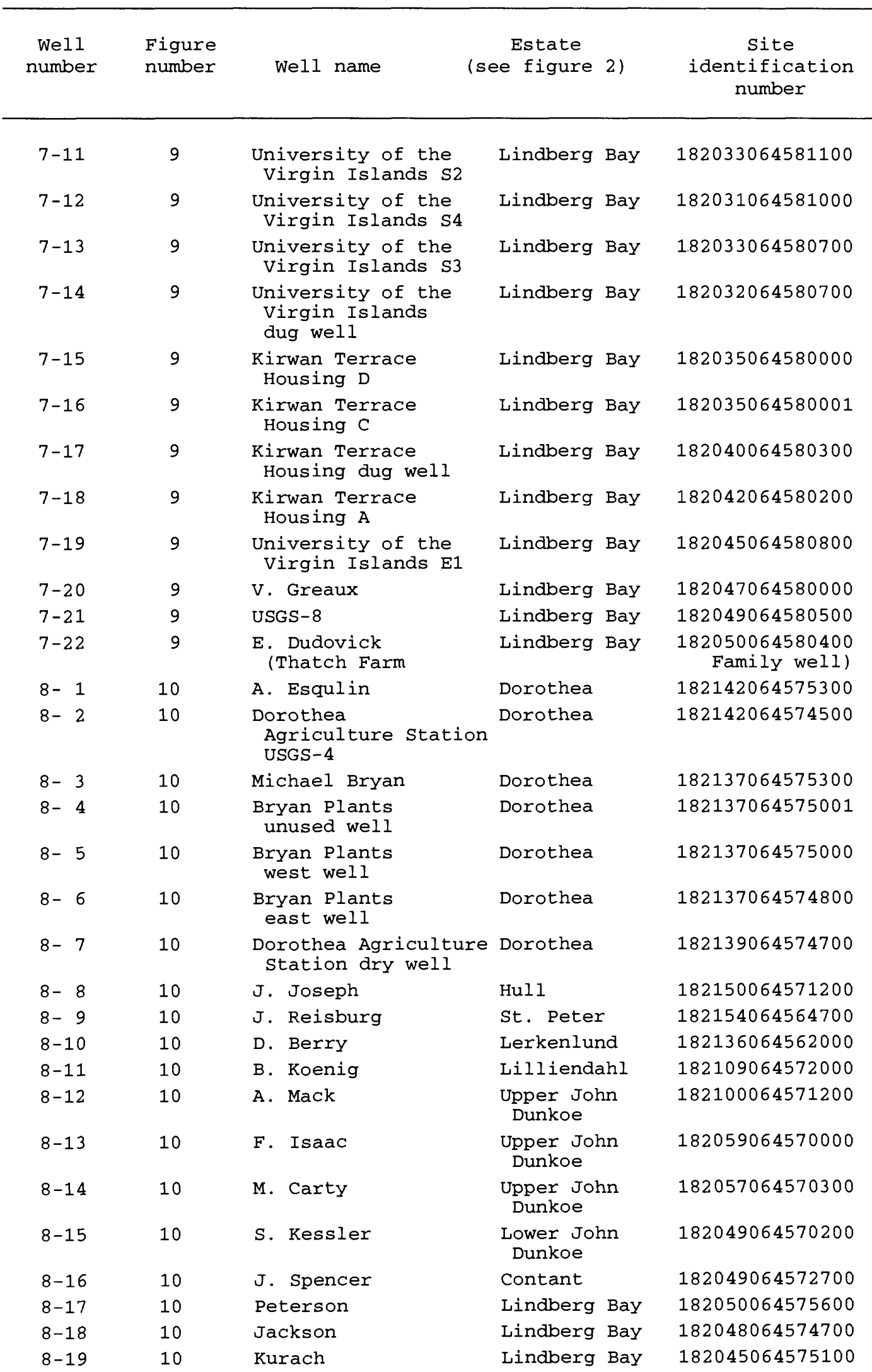


Appendix A. Well names, Estates, and site-identification numbers of wells on St. Thomas, U.S. Virgin Islands--Continued

\begin{tabular}{|c|c|c|c|c|}
\hline $\begin{array}{c}\text { Well } \\
\text { number }\end{array}$ & $\begin{array}{l}\text { Figure } \\
\text { number }\end{array}$ & Well name & $\begin{array}{l}\text { Estate } \\
\text { (see figure 2) }\end{array}$ & $\begin{array}{c}\text { Site } \\
\text { identification } \\
\text { number }\end{array}$ \\
\hline $8-20$ & 10 & W. Smith & Lindberg Bay & 182043064574900 \\
\hline $8-21$ & 10 & S. Joshua & Lindberg Bay & 182043064575300 \\
\hline $8-22$ & 10 & $\begin{array}{l}\text { Kirwan Terrace } \\
\text { Housing } \mathrm{B}\end{array}$ & Lindberg Bay & 182038064575700 \\
\hline $8-23$ & 10 & J. Odato & Lindberg Bay & 182037064575000 \\
\hline $8-24$ & 10 & Delagard & Lindberg Bay & 182035064575300 \\
\hline $8-25$ & 10 & F. El-Haj & Lindberg Bay & 182032064574800 \\
\hline $8-26$ & 10 & $\begin{array}{l}\text { Sunny Mountain } \\
\text { Water }\end{array}$ & Lindberg Bay & 182031064574700 \\
\hline $8-27$ & 10 & $\begin{array}{l}\text { Island Beachcomber } \\
\text { Hotel }\end{array}$ & Lindberg Bay & 182017064575200 \\
\hline $8-28$ & 10 & Rock Crusher Plant & Lindberg Bay & 182024064574200 \\
\hline $8-29$ & 10 & Muhsen/Muhsen & Contant & 182028064573800 \\
\hline $8-30$ & 10 & $\begin{array}{l}\text { W. Turnbull Estate } \\
\text { Contant west well }\end{array}$ & Contant & 182029064573300 \\
\hline $8-31$ & 10 & $\begin{array}{l}\text { W. Turnbull Estate } \\
\text { Contant east well }\end{array}$ & Contant & 182028064573200 \\
\hline $8-32$ & 10 & A. King & Contant & 182027064573300 \\
\hline $8-33$ & 10 & $\begin{array}{l}\text { Virgin Islands } \\
\text { Development Corp. }\end{array}$ & Contant & 182025064573300 \\
\hline $8-34$ & 10 & Supercat laundry & Contant & 182025064573301 \\
\hline $8-35$ & 10 & $\begin{array}{l}\text { Friendly } \\
\text { Supermarket }\end{array}$ & Contant & 182024064573400 \\
\hline $8-36$ & 10 & J. Monsanto & Nisky & 182018064571800 \\
\hline $8-37$ & 10 & E. Quaniang & Contant & 182022064571800 \\
\hline $8-38$ & 10 & F. Esquirdo & Contant & 182023064571100 \\
\hline $8-39$ & 10 & Bamboushay club & Contant & 182024064571100 \\
\hline $8-40$ & 10 & L. Olive & Contant & 182028064571200 \\
\hline $8-41$ & 10 & Aubain & Contant & 182028064571000 \\
\hline $8-42$ & 10 & M. Hughes & Contant & 182036064571500 \\
\hline $8-43$ & 10 & Soto Town & Contant & 182038064571400 \\
\hline $8-44$ & 10 & W. White & Contant & 182042064571400 \\
\hline $8-45$ & 10 & Vanterpool & Contant & 182042064571200 \\
\hline $8-46$ & 10 & Virgin Isle Hotel & $\begin{array}{l}\text { Lower John } \\
\text { Dunkoe }\end{array}$ & 182039064570200 \\
\hline $8-47$ & 10 & H. Richards & Contant & 182029064565800 \\
\hline $8-48$ & 10 & J. Gifft & Contant & 182029064565600 \\
\hline $8-49$ & 10 & Gilmore Enterprises & Contant & 182024064565700 \\
\hline $8-50$ & 10 & Gottlieb Quick Way & Contant & 182024064565200 \\
\hline $8-51$ & 10 & L. Mitchell & Contant & 182026064565200 \\
\hline $8-52$ & 10 & K. Thomas & Contant & 182028064565300 \\
\hline $8-53$ & 10 & V. Hendricks & Contant & 182028064565200 \\
\hline $8-54$ & 10 & Linden Corp. & Altona & 182024064564200 \\
\hline $8-55$ & 10 & $\begin{array}{l}\text { Housing, Parks, } \\
\text { and Recreation/ } \\
\text { Altona north well }\end{array}$ & Altona & 182036064564100 \\
\hline $8-56$ & 10 & $\begin{array}{l}\text { Housing, Parks, } \\
\text { and Recreation/ } \\
\text { Altona south well }\end{array}$ & Altona & 182035064564100 \\
\hline $8-57$ & 10 & H. Lanclos & Anna's Fancy & 182035064563100 \\
\hline
\end{tabular}


Appendix A. Well names, Estates, and site-identification numbers of wells on St. Thomas, U.S. Virgin Islands--Continued

\begin{tabular}{|c|c|c|c|c|}
\hline $\begin{array}{l}\text { Wel1 } \\
\text { number }\end{array}$ & $\begin{array}{l}\text { Figure } \\
\text { number }\end{array}$ & Well name & $\begin{array}{c}\text { Estate } \\
\text { see figure 2) }\end{array}$ & $\begin{array}{c}\text { Site } \\
\text { identification } \\
\text { number }\end{array}$ \\
\hline $8-58$ & 10 & $\begin{array}{l}\text { VI Government/ } \\
\text { Municipal Cemetery }\end{array}$ & Anna's Fancy & 182033064563100 \\
\hline $8-59$ & 10 & C. Christopher & Anna's Fancy & 182037064562700 \\
\hline $8-60$ & 10 & H. Turnbull & $\begin{array}{l}\text { City of } \\
\text { Charlott } \\
\text { Amalie }\end{array}$ & 182035064562100 \\
\hline $8-61$ & 10 & Pure Tropical water & $\begin{array}{l}\text { City of } \\
\text { Charlotte } \\
\text { Amalie }\end{array}$ & 182031064561700 \\
\hline $8-62$ & 10 & F. Magras & $\begin{array}{l}\text { City of } \\
\text { Charlotte } \\
\text { Amalie }\end{array}$ & 182031064561701 \\
\hline $8-63$ & 10 & V. Lettsome & Staabi & 182049064561900 \\
\hline $8-64$ & 10 & $\begin{array}{l}\text { VI Government/ } \\
\text { Barnaber well }\end{array}$ & $\begin{array}{l}\text { City of } \\
\text { Charlotte } \\
\text { Amalie }\end{array}$ & 182047064561000 \\
\hline $8-65$ & 10 & H. Turnbull & $\begin{array}{l}\text { City of } \\
\text { Charlotte } \\
\text { Amalie }\end{array}$ & 182044064561200 \\
\hline $9-1$ & 11 & $\begin{array}{l}\text { Magens Beach } \\
\text { dug well }\end{array}$ & Magens Bay & 182157064552200 \\
\hline $9-2$ & 11 & $\begin{array}{l}\text { Magens Beach } \\
\text { drilled well }\end{array}$ & Magens Bay & 182155064552200 \\
\hline $9-3$ & 11 & Arboretum & Magens Bay & 182136064553100 \\
\hline $9-4$ & 11 & D. Raimer & $\begin{array}{l}\text { St. Joseph } \\
\text { \& Rosendal }\end{array}$ & 182146064550500 \\
\hline $9-5$ & 11 & J. Ladee & $\begin{array}{l}\text { St. Joseph } \\
\text { \& Rosendal }\end{array}$ & 182143064545900 \\
\hline $9-6$ & 11 & Magens Point Hotel & $\begin{array}{l}\text { St. Joseph } \\
\text { \& Rosendal }\end{array}$ & 182141064545700 \\
\hline $9-7$ & 11 & St. Thomas Dairy E & $\begin{array}{l}\text { St. Joseph } \\
\text { \& Rosendal }\end{array}$ & 182142064545600 \\
\hline $9-8$ & 11 & St. Thomas Dairy F & $\begin{array}{l}\text { St. Joseph } \\
\text { \& Rosendal }\end{array}$ & 182141064545300 \\
\hline $9-9$ & 11 & St. Thomas Dairy A & $\begin{array}{l}\text { St. Joseph } \\
\text { \& Rosendal }\end{array}$ & 182142064545000 \\
\hline $9-10$ & 11 & St. Thomas Dairy B & $\begin{array}{l}\text { St. Joseph } \\
\text { \& Rosendal }\end{array}$ & 182141064545000 \\
\hline $9-11$ & 11 & $\begin{array}{l}\text { st. Thomas Dairy } \\
\text { supply well }\end{array}$ & Lovenlund & 182149064544800 \\
\hline $9-12$ & 11 & $\begin{array}{l}\text { St. Thomas Dairy } \\
\text { dug well }\end{array}$ & $\begin{array}{l}\text { St. Joseph } \\
\& \text { Rosendal }\end{array}$ & 182148064544700 \\
\hline $9-13$ & 11 & Mahogany Run 1 & Lovenlund & 182150064544500 \\
\hline $9-14$ & 11 & Mahogany Run 2 & Lovenlund & 182150064544100 \\
\hline $9-15$ & 11 & Mahogany Run 3 & Lovenlund & 182148064543900 \\
\hline $9-16$ & 11 & Mahogany Run 3A & Lovenlund & 182148064543800 \\
\hline $9-17$ & 11 & Mahogany Run 3B & Lovenlund & 182147064543700 \\
\hline $9-18$ & 11 & Mahogany Run 4 & Lovenlund & 182146064543600 \\
\hline $9-19$ & 11 & Mahogany Run 5 & Lovenlund & 182145064543500 \\
\hline $9-20$ & 11 & Mahogany Run $5 a$ & Lovenlund & 182144064543400 \\
\hline $9-21$ & 11 & Mahogany Run 7 & Lovenlund & 182143064543300 \\
\hline $9-22$ & 11 & Mahogany Run 8 & Lovenlund & 182142064543300 \\
\hline
\end{tabular}


Appendix A. Well names, Estates, and site-identification numbers of wells on St. Thomas, U.S. Virgin Islands--Continued

\begin{tabular}{|c|c|c|c|c|}
\hline $\begin{array}{l}\text { Wel1 } \\
\text { number }\end{array}$ & $\begin{array}{l}\text { Figure } \\
\text { number }\end{array}$ & Well name & $\begin{array}{c}\text { Estate } \\
\text { see figure 2) }\end{array}$ & $\begin{array}{c}\text { Site } \\
\text { identification } \\
\text { number }\end{array}$ \\
\hline $9-23$ & 11 & Mahogany Run 9 & Lovenlund & 182141064543300 \\
\hline $9-24$ & 11 & $\begin{array}{l}\text { Mahogany Run dug } \\
\text { well }\end{array}$ & Lovenlund & 182140064543200 \\
\hline $9-25$ & 11 & Mahogany Run 11 & Lovenlund & 182140064543000 \\
\hline $9-26$ & 11 & $\begin{array}{l}\text { Mahogany Run } 10 / \\
\text { St. Thomas Dairy } \\
\text { USGS-3 }\end{array}$ & Lovenlund & 182138064543101 \\
\hline $9-27$ & 11 & Mahogany Run 12 & Lovenlund & 182138064543000 \\
\hline $9-28$ & 11 & Mahogany Run 14 & Lovenlund & 182135064542700 \\
\hline $9-29$ & 11 & Mahogany Run 14A & Lovenlund & 182135064542701 \\
\hline $9-30$ & 11 & Mahogany Run 15 & Lovenlund & 182138064543100 \\
\hline $9-31$ & 11 & Mahogany Run 16 & Lovenlund & 182138064542500 \\
\hline $9-32$ & 11 & Mahogany Run 17 & Lovenlund & 182136064541900 \\
\hline $9-33$ & 11 & F. McConnel & Canaan & 182131064551300 \\
\hline $9-34$ & 11 & A. Raimer & $\begin{array}{l}\text { St. Joseph } \\
\text { \& Rosendal }\end{array}$ & 182118064544900 \\
\hline $9-35$ & 11 & Moorehead & $\begin{array}{l}\text { St. Joseph } \\
\text { \& Rosendal }\end{array}$ & 182117064544600 \\
\hline $9-36$ & 11 & P. Clooney & Wintberg & 182111064542500 \\
\hline $9-37$ & 11 & A. Bornn & Wintberg & 182105064542100 \\
\hline $9-38$ & 11 & E. Lyons & Wintberg & 182103064542400 \\
\hline $9-39$ & 11 & $\begin{aligned} \text { National Park } \\
\text { Service USGS-5 }\end{aligned}$ & Wintberg & 182059064541200 \\
\hline $9-40$ & 11 & E. O'Brien & Mafolie & 182113064555600 \\
\hline $9-41$ & 11 & $\begin{array}{l}\text { VI Government/ } \\
\text { Department of } \\
\text { Education }\end{array}$ & $\begin{array}{l}\text { Litton's } \\
\text { Fancy }\end{array}$ & 182046064555500 \\
\hline $9-42$ & 11 & $\begin{array}{l}\text { VI Government/near } \\
\text { Department of } \\
\text { Education }\end{array}$ & $\begin{array}{l}\text { Litton's } \\
\text { Fancy }\end{array}$ & 182046064555300 \\
\hline $9-43$ & 11 & $\begin{array}{l}\text { VI Government/ } \\
\text { Wishing well }\end{array}$ & $\begin{array}{l}\text { City of } \\
\text { Charlotte } \\
\text { Amalie }\end{array}$ & 182037064555900 \\
\hline $9-44$ & 11 & $\begin{array}{l}\text { VI Government/ } \\
\text { Back street }\end{array}$ & $\begin{array}{l}\text { City of } \\
\text { Charlotte } \\
\text { Amalie }\end{array}$ & 182040064555400 \\
\hline $9-45$ & 11 & $\begin{array}{l}\text { Knud Hansen Complex } \\
\text { drilled well }\end{array}$ & $\begin{array}{l}\text { Hospital } \\
\text { Ground }\end{array}$ & 182053064553600 \\
\hline $9-46$ & 11 & $\begin{array}{l}\text { Knud Hansen Complex } \\
\text { dug well }\end{array}$ & $\begin{array}{l}\text { Hospital } \\
\text { Ground }\end{array}$ & 182050064553500 \\
\hline $9-47$ & 11 & B. George & $\begin{array}{l}\text { Hospital } \\
\text { Ground }\end{array}$ & 182049064552900 \\
\hline $9-48$ & 11 & D. Michael & $\begin{array}{l}\text { City of } \\
\text { Charlotte }\end{array}$ & 182042064553600 \\
\hline $9-49$ & 11 & W. Turnbull & $\begin{array}{l}\text { City of } \\
\text { Charlotte } \\
\text { Amalie }\end{array}$ & 182042064553400 \\
\hline $9-50$ & 11 & $\begin{array}{l}\text { VI Government/ } \\
\text { dug well by } \\
\text { Wet willy's }\end{array}$ & $\begin{array}{l}\text { City of } \\
\text { Charlotte } \\
\text { Amalie }\end{array}$ & 182035064553500 \\
\hline $9-51$ & 11 & $\begin{array}{l}\text { Bluebeards } \\
\text { Castle Hotel } 2\end{array}$ & $\begin{array}{l}\text { City of } \\
\text { Charlotte } \\
\text { Amalie }\end{array}$ & 182033064553000 \\
\hline
\end{tabular}


Appendix A. Well names, Estates, and site-identification numbers of wells on St. Thomas, U.S. Virgin Islands--Continued

\begin{tabular}{|c|c|c|c|c|}
\hline $\begin{array}{l}\text { Well } \\
\text { number }\end{array}$ & $\begin{array}{l}\text { Figure } \\
\text { number }\end{array}$ & Well name & $\begin{array}{l}\text { Estate } \\
\text { ee figure 2) }\end{array}$ & $\begin{array}{c}\text { Site } \\
\text { identification } \\
\text { number }\end{array}$ \\
\hline $9-52$ & 11 & $\begin{array}{l}\text { Bluebeards } \\
\text { Castle Hotel } 1\end{array}$ & $\begin{array}{l}\text { City of } \\
\text { Charlotte } \\
\text { Amalie }\end{array}$ & 182030064552700 \\
\hline $9-53$ & 11 & Barbel Assoc. & Thomas & 182030064551800 \\
\hline $9-54$ & 11 & $\begin{array}{l}\text { VI Government/ } \\
\text { Department of } \\
\text { Human Services }\end{array}$ & Thomas & 182030064551500 \\
\hline $9-55$ & 11 & Hendricks & Thomas & 182029064551200 \\
\hline $9-56$ & 11 & C. Jensen & Thomas & 182029064551000 \\
\hline $9-57$ & 11 & Burnette Towers & Thomas & 182028064551000 \\
\hline $9-58$ & 11 & I. Estrill & Thomas & 182029064550500 \\
\hline $9-59$ & 11 & One stop & Thomas & 182033064550500 \\
\hline $9-60$ & 11 & $\begin{array}{l}\text { Lockhart School } \\
\text { northwest corner }\end{array}$ & Thomas & 182037064550500 \\
\hline $9-61$ & 11 & $\begin{array}{l}\text { Grade School } \\
\text { well } 3\end{array}$ & Thomas & 182038064550300 \\
\hline $9-62$ & 11 & $\begin{array}{l}\text { Water and Power } \\
\text { Authority/ } \\
\text { Wheatly Skill } \\
\text { Center west well }\end{array}$ & Thomas & 182041064550500 \\
\hline $9-63$ & 11 & $\begin{array}{l}\text { Water and Power } \\
\text { Authority/ } \\
\text { Wheatly Skill } \\
\text { Center east well }\end{array}$ & Thomas & 182041064550300 \\
\hline $9-64$ & 11 & $\begin{array}{l}\text { Water and Power } \\
\text { Authority/ } \\
\text { WAPA pump station }\end{array}$ & Thomas & 182039064550000 \\
\hline $9-65$ & 11 & $\begin{array}{l}\text { Lockhart School } \\
\text { northeast corner }\end{array}$ & Thomas & 182038064550000 \\
\hline $9-66$ & 11 & $\begin{array}{l}\text { Lockhart School } \\
\text { playground }\end{array}$ & Thomas & 182033064550000 \\
\hline $9-67$ & 11 & G. Blackhal & Thomas & 182029064550100 \\
\hline $9-68$ & 11 & Hallpicke & Thomas & 182028064550200 \\
\hline $9-69$ & 11 & Rodgers & Thomas & 182025064550300 \\
\hline $9-70$ & 11 & G. Hodge & Thomas & 182026064550400 \\
\hline $9-71$ & 11 & J. Blyden & Thomas & 182025064550800 \\
\hline $9-72$ & 11 & Moore & Thomas & 182025064550600 \\
\hline $9-73$ & 11 & L. Mathias & Thomas & 182023064550600 \\
\hline $9-74$ & 11 & E. Radsatt & Thomas & 182023064550601 \\
\hline $9-75$ & 11 & Walters & Thomas & 182022064550500 \\
\hline $9-76$ & 11 & A. Lima & Thomas & 182023064550300 \\
\hline $9-77$ & 11 & Yeppsen & Thomas & 182024064550100 \\
\hline $9-78$ & 11 & Yacht Haven Hotel & Thomas & 182017064551300 \\
\hline $9-79$ & 11 & St. Thomas Mall Inc. & Thomas & 182020064550400 \\
\hline $9-80$ & 11 & $\begin{array}{l}\text { W. Turnbull Estate } \\
\text { Thomas west well }\end{array}$ & Thomas & 182014064550000 \\
\hline $9-81$ & 11 & $\begin{array}{l}\text { W. Turnbull Estate } \\
\text { Thomas east well }\end{array}$ & Thomas & 182015064545400 \\
\hline $9-82$ & 11 & $\begin{array}{l}\text { Wheatly } \\
\text { Shopping center }\end{array}$ & Thomas & 182024064545000 \\
\hline $9-83$ & 11 & G. Melchoir & Thomas & 182028064545300 \\
\hline
\end{tabular}


Appendix A. Well names, Estates, and site-identification numbers of wells on St. Thomas, U.S. Virgin Islands--Continued

\begin{tabular}{|c|c|c|c|c|}
\hline $\begin{array}{l}\text { Wel1 } \\
\text { number }\end{array}$ & $\begin{array}{l}\text { Figure } \\
\text { number }\end{array}$ & Well name & $\begin{array}{l}\text { Estate } \\
\text { ee figure 2) }\end{array}$ & $\begin{array}{c}\text { Site } \\
\text { identification } \\
\text { number }\end{array}$ \\
\hline $9-84$ & 11 & $\begin{array}{l}\text { St. Thomas } \\
\text { Hospital } 3\end{array}$ & Thomas & 182031064545500 \\
\hline $9-85$ & 11 & $\begin{array}{l}\text { St. Thomas } \\
\text { Hospital } 2\end{array}$ & Thomas & 182033064545500 \\
\hline $9-86$ & 11 & $\begin{array}{l}\text { St. Thomas } \\
\text { Hospital } 1\end{array}$ & Thomas & 182036064545600 \\
\hline $9-87$ & 11 & $\begin{array}{l}\text { Lockhart Estate } \\
\text { Thomas north well }\end{array}$ & Thomas & 182037064544600 \\
\hline $9-88$ & 11 & Daily News & Thomas & 182035064544800 \\
\hline $9-89$ & 11 & $\begin{array}{l}\text { Virgin Islands } \\
\text { Medical Association }\end{array}$ & Thomas & 182033064544800 \\
\hline $9-90$ & 11 & $\begin{array}{l}\text { Lockhart Estate } \\
\text { Thomas south well }\end{array}$ & Thomas & 182032064544600 \\
\hline $9-91$ & 11 & Lockhart/ Melchoir & Thomas & 182030064544800 \\
\hline $9-92$ & 11 & T. Frett & Thomas & 182014064544100 \\
\hline $9-93$ & 11 & L. Barbei & Raphune & 182009064543300 \\
\hline $9-94$ & 11 & Villa Blanca Hotel & Thomas & 182021064543400 \\
\hline $9-95$ & 11 & Lockhart dug well & Raphune & 182017064542300 \\
\hline $9-96$ & 11 & New Hernhut Mission & New Hernhut & 182002064540700 \\
\hline $9-97$ & 11 & Lockhart 5 & Donoe & 182015064540000 \\
\hline $9-98$ & 11 & Lockhart 4 & Donoe & 182016064540000 \\
\hline $9-99$ & 11 & Lockhart 3 & Donoe & 182016064535900 \\
\hline $10-1$ & 12 & Mandahl Beach & Mandahl & 182141064535000 \\
\hline $10-2$ & 12 & $\begin{array}{l}\text { VI Government/ } \\
\text { Mandah1 Beach }\end{array}$ & Mandah1 & 182137064533800 \\
\hline $10-3$ & 12 & V. Wallace & Mandah1 & 182124064532900 \\
\hline $10-4$ & 12 & Peace Corps School & Mandah1 & 182125064535700 \\
\hline $10-5$ & 12 & $\begin{array}{l}\text { Mandahl } \\
\text { Homestead well }\end{array}$ & Mandahl & 182122064535900 \\
\hline $10-6$ & 12 & Demitry & $\begin{array}{l}\text { Anna's } \\
\text { Retreat }\end{array}$ & 182042064533400 \\
\hline $10-7$ & 12 & Alpha Leonard & $\begin{array}{l}\text { Anna's } \\
\text { Retreat }\end{array}$ & 182038064533300 \\
\hline $10-8$ & 12 & $\begin{array}{l}\text { VI Government/ } \\
\text { near treatment } \\
\text { plant }\end{array}$ & $\begin{array}{l}\text { Anna's } \\
\text { Retreat }\end{array}$ & 182039064534400 \\
\hline $10-9$ & 12 & $\begin{array}{l}\text { VI Government/ } \\
\text { Donoe } 3\end{array}$ & Donoe & 182029064535200 \\
\hline $10-10$ & 12 & M. Bryan 2 & Donoe & 182026064535800 \\
\hline $10-11$ & 12 & M. Bryan 3 & Donoe & 182025064535800 \\
\hline $10-12$ & 12 & M. Bryan 1 & Donoe & 182024064535800 \\
\hline $10-13$ & 12 & Lockhart 1 & Donoe & 182018064535900 \\
\hline $10-14$ & 12 & Lockhart 2 & Donoe & 182017064535900 \\
\hline $10-15$ & 12 & I. Kirkerterp & Hoffman & 182012064535400 \\
\hline $10-16$ & 12 & J. Baugh & $\begin{array}{l}\text { Charlotte } \\
\text { Amalie }\end{array}$ & 182017064534900 \\
\hline $10-17$ & 12 & Pedersen & Hoffman & 182009064534500 \\
\hline $10-18$ & 12 & A. Sharp & $\begin{array}{l}\text { Charlotte } \\
\text { Amalie }\end{array}$ & 182010064534200 \\
\hline $10-19$ & 12 & E. Eglin & $\begin{array}{l}\text { Charlotte } \\
\text { Amalie }\end{array}$ & 182011064534000 \\
\hline
\end{tabular}


Appendix A. Well names, Estates, and site-identification numbers of wells on St. Thomas, U.S. Virgin Islands--Continued

\begin{tabular}{|c|c|c|c|c|}
\hline $\begin{array}{l}\text { We11 } \\
\text { number }\end{array}$ & $\begin{array}{l}\text { Figure } \\
\text { number }\end{array}$ & Well name & $\begin{array}{c}\text { Estate } \\
\text { (see figure 2) }\end{array}$ & $\begin{array}{c}\text { Site } \\
\text { identification } \\
\text { number }\end{array}$ \\
\hline $10-20$ & 12 & Harthman dug well & $\begin{array}{l}\text { Charlotte } \\
\text { Amalie }\end{array}$ & 182023064533800 \\
\hline $10-21$ & 12 & $\begin{array}{l}\text { Harthman } \\
\text { domestic well }\end{array}$ & $\begin{array}{l}\text { Charlotte } \\
\text { Amalie }\end{array}$ & 182019064533500 \\
\hline $10-22$ & 12 & VITELCO & $\begin{array}{l}\text { Charlotte } \\
\text { Amalie }\end{array}$ & 182018064533200 \\
\hline $10-23$ & 12 & Gassett Motors 1 & $\begin{array}{l}\text { Charlotte } \\
\text { Amalie }\end{array}$ & 182017064533000 \\
\hline $10-24$ & 12 & Gassett Motors 2 & $\begin{array}{l}\text { Charlotte } \\
\text { Amalie }\end{array}$ & 182018064533100 \\
\hline $10-25$ & 12 & Rodriguez Auto 2 & $\begin{array}{l}\text { Charlotte } \\
\text { Amalie }\end{array}$ & 182016064532900 \\
\hline $10-26$ & 12 & Rodriguez Auto 3 & $\begin{array}{l}\text { Charlotte } \\
\text { Amalie }\end{array}$ & 182015064533000 \\
\hline $10-27$ & 12 & Rodriguez Auto 4 & $\begin{array}{l}\text { Charlotte } \\
\text { Amalie }\end{array}$ & 182014064532900 \\
\hline $10-28$ & 12 & Fort Mylner & $\begin{array}{l}\text { Charlotte } \\
\text { Amalie }\end{array}$ & 182010064532900 \\
\hline $10-29$ & 12 & Versailles Assoc. & $\begin{array}{l}\text { Charlotte } \\
\text { Amalie }\end{array}$ & 182009064532800 \\
\hline $10-30$ & 12 & Delagarde & $\begin{array}{l}\text { Charlotte } \\
\text { Amalie }\end{array}$ & 182001064530800 \\
\hline $10-31$ & 12 & Matthias & $\begin{array}{l}\text { Charlotte } \\
\text { Amalie }\end{array}$ & 182008064531400 \\
\hline $10-32$ & 12 & L. Smith & $\begin{array}{l}\text { Charlotte } \\
\text { Amalie }\end{array}$ & 182009064531300 \\
\hline $10-33$ & 12 & C. Hodge & $\begin{array}{l}\text { Anna's } \\
\text { Retreat }\end{array}$ & 182012064531400 \\
\hline $10-34$ & 12 & E. Steel & $\begin{array}{l}\text { Anna's } \\
\text { Retreat }\end{array}$ & 182016064532000 \\
\hline $10-35$ & 12 & O. Harvey & $\begin{array}{l}\text { Anna's } \\
\text { Retreat }\end{array}$ & 182017064532200 \\
\hline $10-36$ & 12 & $\begin{array}{l}\text { Harthman near } \\
\text { Gassett Motors }\end{array}$ & $\begin{array}{l}\text { Charlotte } \\
\text { Amalie }\end{array}$ & 182018064532800 \\
\hline $10-37$ & 12 & Crusher Well & $\begin{array}{l}\text { Charlotte } \\
\text { Amalie }\end{array}$ & 182021064532500 \\
\hline $10-38$ & 12 & G. Eglin 1 & $\begin{array}{l}\text { Anna's } \\
\text { Retreat }\end{array}$ & 182020064532000 \\
\hline $10-39$ & 12 & G. Eglin 3 & $\begin{array}{l}\text { Anna's } \\
\text { Retreat }\end{array}$ & 182019064531900 \\
\hline $10-40$ & 12 & G. Eglin 2 & $\begin{array}{l}\text { Anna's } \\
\text { Retreat }\end{array}$ & 182020064531900 \\
\hline $10-41$ & 12 & Tom Cat laundry & $\begin{array}{l}\text { Anna's } \\
\text { Retreat }\end{array}$ & 182021064531900 \\
\hline $10-42$ & 12 & $\begin{array}{l}\text { VI Government/ } \\
\text { near Church } \\
\text { of God Holiness }\end{array}$ & $\begin{array}{l}\text { Anna's } \\
\text { Retreat }\end{array}$ & 182021064532000 \\
\hline $10-43$ & 12 & $\begin{array}{l}\text { Harthman Estate } \\
\text { well USGS-25 }\end{array}$ & $\begin{array}{l}\text { Charlotte } \\
\text { Amalie }\end{array}$ & 182027064532900 \\
\hline $10-44$ & 12 & $\begin{array}{l}\text { Church of } \\
\text { God Holiness }\end{array}$ & $\begin{array}{l}\text { Anna's } \\
\text { Retreat }\end{array}$ & 182025064532300 \\
\hline $10-45$ & 12 & Four Winds Plaza 2 & $\begin{array}{l}\text { Anna's } \\
\text { Retreat }\end{array}$ & 182027064531900 \\
\hline
\end{tabular}


Appendix A. Well names, Estates, and site-identification numbers of wells on St. Thomas, U.S. Virgin Islands--Continued

\begin{tabular}{|c|c|c|c|c|}
\hline $\begin{array}{c}\text { Well } \\
\text { number }\end{array}$ & $\begin{array}{l}\text { Figure } \\
\text { number }\end{array}$ & Well name & $\begin{array}{l}\text { Estate } \\
\text { ee figure 2) }\end{array}$ & $\begin{array}{c}\text { Site } \\
\text { identification } \\
\text { number }\end{array}$ \\
\hline $10-46$ & 12 & Four Winds Plaza 1 & $\begin{array}{l}\text { Anna's } \\
\text { Retreat }\end{array}$ & 182027064531800 \\
\hline $10-47$ & 12 & E. Tillet & $\begin{array}{l}\text { Anna's } \\
\text { Retreat }\end{array}$ & 182029064531500 \\
\hline $10-48$ & 12 & Ramsey Motors & $\begin{array}{l}\text { Anna's } \\
\text { Retreat }\end{array}$ & 182036064531600 \\
\hline $10-49$ & 12 & $\begin{array}{l}\text { Virgin Islands } \\
\text { Housing } \\
\text { Authority } 1\end{array}$ & $\begin{array}{l}\text { Anna's } \\
\text { Retreat }\end{array}$ & 182037064531100 \\
\hline $10-50$ & 12 & $\begin{array}{l}\text { Virgin Islands } \\
\text { Housing } \\
\text { Authority } 2\end{array}$ & $\begin{array}{l}\text { Anna's } \\
\text { Retreat }\end{array}$ & 182038064531200 \\
\hline $10-51$ & 12 & $\begin{array}{l}\text { Virgin Islands } \\
\text { Housing } \\
\text { Authority } 3\end{array}$ & $\begin{array}{l}\text { Anna's } \\
\text { Retreat }\end{array}$ & 182048064531400 \\
\hline $10-52$ & 12 & $\begin{array}{l}\text { Virgin Islands } \\
\text { Housing } \\
\text { Authority } 4\end{array}$ & $\begin{array}{l}\text { Anna's } \\
\text { Retreat }\end{array}$ & 182049064530900 \\
\hline $10-53$ & 12 & $\begin{array}{l}\text { C. F. Rosenberg } \\
\text { USGS-2 }\end{array}$ & Frydendahl & 182048064524000 \\
\hline $10-54$ & 12 & Super Pool Resturant & Frydendahl & 182049064524000 \\
\hline $10-55$ & 12 & Smith Bay Center & Frydendahl & 182050064523800 \\
\hline $10-56$ & 12 & A. Levia & Frydendahl & 182051064523500 \\
\hline $10-57$ & 12 & U. Pilgrim & Frydendahl & 182054064523400 \\
\hline $10-58$ & 12 & $\begin{array}{c}\text { Fat Man's / } \\
\text { C. Husband }\end{array}$ & Frydendah1 & 182053064522900 \\
\hline $10-59$ & 12 & R. Mercer & Frydendah1 & 182100064522300 \\
\hline $10-60$ & 12 & $\begin{array}{l}\text { Stouffer Grand } \\
\text { Beach Hotel A }\end{array}$ & Frydendah1 & 182057064521600 \\
\hline $10-61$ & 12 & $\begin{array}{l}\text { Stouffer Grand } \\
\text { Beach Hotel B }\end{array}$ & Fryndenahl & 182056064521700 \\
\hline $10-62$ & 12 & Leonard & Frydendahl & 182055064522100 \\
\hline $10-63$ & 12 & A. Leonard 2 & Frydendahl & 182054064522000 \\
\hline $10-64$ & 12 & A. Leonard 1 & Frydendah1 & 182053064522100 \\
\hline $10-65$ & 12 & $S \& S$ Service & Smith Bay & 182050064521800 \\
\hline $10-66$ & 12 & E. Francis & Smith Bay & 182046064522300 \\
\hline $10-67$ & 12 & $\begin{array}{l}\text { Stouffer Grand } \\
\text { Beach Hotel C }\end{array}$ & Frydendah1 & 182058064520600 \\
\hline $10-68$ & 12 & $\begin{array}{l}\text { Stouffer Grand } \\
\text { Beach Hotel D }\end{array}$ & Frydendah1 & 182057064520600 \\
\hline $10-69$ & 12 & $\begin{array}{l}\text { Stouffer Grand } \\
\text { Beach Hotel E }\end{array}$ & Frydendah1 & 182055064520600 \\
\hline $10-70$ & 12 & Martin & Smith Bay & 182043064521000 \\
\hline $10-71$ & 12 & $\begin{array}{l}\text { Lindquist/VI } \\
\text { Government }\end{array}$ & Smith Bay & 182041064521100 \\
\hline $10-72$ & 12 & Sprauve & Smith Bay & 182037064521200 \\
\hline $10-73$ & 12 & $\begin{array}{l}\text { Point Pleasant } \\
\text { Resort } 1\end{array}$ & Smith Bay & 182041064520300 \\
\hline $10-74$ & 12 & Johnson & Smith Bay & 182040064520400 \\
\hline $11-1$ & 13 & $\begin{array}{l}\text { Point Pleasant } \\
\text { Resort } 2\end{array}$ & Smith Bay & 182040064520000 \\
\hline $11-2$ & 13 & S. Harthman Heirs & Smith Bay & 182026064513400 \\
\hline
\end{tabular}


Appendix A. Well names, Estates, and site-identification numbers of wells on St. Thomas, U.S. Virgin Islands--Continued

\begin{tabular}{|c|c|c|c|c|}
\hline $\begin{array}{c}\text { Wel1 } \\
\text { number }\end{array}$ & $\begin{array}{l}\text { Figure } \\
\text { number }\end{array}$ & Well name & $\begin{array}{c}\text { Estate } \\
\text { (see figure 2) }\end{array}$ & $\begin{array}{c}\text { Site } \\
\text { identification } \\
\text { number }\end{array}$ \\
\hline $11-3$ & 13 & Lindquist & Smith Bay & 182018064512800 \\
\hline $11-4$ & 13 & $\begin{array}{l}\text { Sapphire Bay Condo } \\
\text { West-supply well }\end{array}$ & Smith Bay & 182018064511800 \\
\hline $11-5$ & 13 & $\begin{array}{l}\text { Sapphire Bay Condo } \\
\text { West-east well }\end{array}$ & Smith Bay & 182017064511201 \\
\hline $11-6$ & 13 & $\begin{array}{l}\text { Sapphire Bay Condo } \\
\text { West-west well }\end{array}$ & Smith Bay & 182017064511200 \\
\hline $11-7$ & 13 & $\begin{array}{l}\text { S. Harthman Heirs } \\
\text { USGS-23 }\end{array}$ & Smith Bay & 182001064510900 \\
\hline $14-1$ & 16 & M. Carter & Bakkeroe & 181951064544900 \\
\hline $14-2$ & 16 & Frenchman's Reef 3 & Bakkeroe & 181929064551700 \\
\hline $14-3$ & 16 & Frenchman's Reef 1 & Bakkeroe & 181928064551700 \\
\hline $14-4$ & 16 & $\begin{array}{l}\text { Frenchman's Reef } \\
\text { maintenance }\end{array}$ & Bakkeroe & 181925064552000 \\
\hline $14-5$ & 16 & Morning Star & Bakkeroe & 181921064550500 \\
\hline $14-6$ & 16 & A. Williamson & Frenchman Bay & 181929064544200 \\
\hline $14-7$ & 16 & Antilles School & Frenchman Bay & 181930064543900 \\
\hline $14-8$ & 16 & Limetree Hotel & Frenchman Bay & 181912064545100 \\
\hline $14-9$ & 16 & A. Halpern & Frenchman Bay & 181911064544200 \\
\hline $14-10$ & 16 & F. Samad & Frenchman Bay & 181905064543600 \\
\hline $14-11$ & 16 & F. Mercetelli & Frenchman Bay & 181901064542300 \\
\hline $14-12$ & 16 & $\begin{array}{l}\text { Hydrofarms } \\
\text { north well }\end{array}$ & Bolongo & 181919064540700 \\
\hline $14-13$ & 16 & $\begin{array}{l}\text { Hydrofarms } \\
\text { south well }\end{array}$ & Bolongo & 181918064540600 \\
\hline $15-1$ & 17 & A. Joseph & Bolongo & 181914064535700 \\
\hline $15-2$ & 17 & E. Cherian & Bolongo & 181912064535700 \\
\hline $15-3$ & 17 & R. Greenberg & Bolongo & 181913064535400 \\
\hline $15-4$ & 17 & McGowen & Bolongo & 181911064535500 \\
\hline $15-5$ & 17 & Charlie's Trucking & Bolongo & 181911064540000 \\
\hline $15-6$ & 17 & R. Moran & Bolongo & 181903064535100 \\
\hline $15-7$ & 17 & G. Black & Bolongo & 181901064535100 \\
\hline $15-8$ & 17 & Bolongo Bay Hotel & Bolongo & 181857064535400 \\
\hline $15-9$ & 17 & $\begin{array}{l}\text { Bolongo Bay } \\
\text { Hotel supply well }\end{array}$ & Bolongo & 181855064535200 \\
\hline $15-10$ & 17 & F. Vialet & Bovoni & 181855064534000 \\
\hline $15-11$ & 17 & Bovoni landfill & Bovoni & 181857064530300 \\
\hline $15-12$ & 17 & R. Charles & Bovoni & 181903064531200 \\
\hline $15-13$ & 17 & $\begin{array}{l}\text { Bovoni Baptist } \\
\text { Mission }\end{array}$ & Bovoni & 181910064531400 \\
\hline $15-14$ & 17 & $\begin{array}{l}\text { Bovoni Baptist } \\
\text { Church }\end{array}$ & Bovoni & 181910064531500 \\
\hline $15-15$ & 17 & Thomasville coop 1 & Bovoni & 181917064532400 \\
\hline $15-16$ & 17 & Thomasville coop 2 & Bovoni & 181919064532200 \\
\hline $15-17$ & 17 & Stevens & Bovoni & 181922064532200 \\
\hline $15-18$ & 17 & A. Lima 4 & Bovoni & 181917064531400 \\
\hline $15-19$ & 17 & A. Lima 2 & Bovoni & 181915064531300 \\
\hline $15-20$ & 17 & A. Lima 1 & Bovoni & 181914064531300 \\
\hline $15-21$ & 17 & A. Lima 3 & Bovoni & 181916064531200 \\
\hline
\end{tabular}


Appendix A. Well names, Estates, and site-identification numbers of wells on St. Thomas, U.S. Virgin Islands--Continued

\begin{tabular}{|c|c|c|c|c|}
\hline $\begin{array}{l}\text { Well } \\
\text { number }\end{array}$ & $\begin{array}{l}\text { Figure } \\
\text { number }\end{array}$ & Well name & $\begin{array}{l}\text { Estate } \\
\text { (see figure 2) }\end{array}$ & $\begin{array}{c}\text { Site } \\
\text { identification } \\
\text { number }\end{array}$ \\
\hline $15-22$ & 17 & A. Lima 5 & Bovoni & 181915064531000 \\
\hline $15-23$ & 17 & A. Lima 6 & Bovoni & 181913064530900 \\
\hline $15-24$ & 17 & A. Lima 7 & Bovoni & 181912064531000 \\
\hline $15-25$ & 17 & M. Lima & Bovoni & 181910064530800 \\
\hline $15-26$ & 17 & Lima superette & Bovoni & 181910064530600 \\
\hline $15-27$ & 17 & Industrious & Bovoni & 181923064525800 \\
\hline $15-28$ & 17 & Dench & Mariendahl & 181945064525500 \\
\hline $15-29$ & 17 & $\begin{array}{l}\text { Poly Caribe/ } \\
\text { Devcon } 1\end{array}$ & Mariendahl & 181940064525200 \\
\hline $15-30$ & 17 & $\begin{array}{l}\text { Poly Caribe/ } \\
\text { Devcon } 2\end{array}$ & Mariendahl & 181938064525100 \\
\hline $15-31$ & 17 & $\begin{array}{l}\text { Poly Caribe/ } \\
\text { Devcon } 3\end{array}$ & Mariendahl & 181938064525000 \\
\hline $15-32$ & 17 & $\begin{array}{l}\text { Poly Caribe/ } \\
\text { Devcon } 4\end{array}$ & Mariendahl & 181937064524900 \\
\hline $15-33$ & 17 & Vessep & Mariendahl & 181936064524700 \\
\hline $15-34$ & 17 & $\begin{array}{l}\text { VI water and Power } \\
\text { Authority/Dede } \\
\text { well - USGS-11 }\end{array}$ & Mariendahl & 181935064524400 \\
\hline $15-35$ & 17 & Lantern laundry & Mariendahl & 181935064524000 \\
\hline $15-36$ & 17 & $\begin{array}{l}\text { VI Government / dug } \\
\text { well near Abattoir }\end{array}$ & Nadir & 181920064523900 \\
\hline $15-37$ & 17 & $\begin{array}{l}\text { VI Government/ } \\
\text { Abattoir east well }\end{array}$ & Nadir & 181920064524200 \\
\hline $15-38$ & 17 & Humane Society & Nadir & 181921064524100 \\
\hline $15-39$ & 17 & S. Rollins & Nadir & 181917064524200 \\
\hline $15-40$ & 17 & I. Lake & Nadir & 181916064524100 \\
\hline $15-41$ & 17 & Fleming & Nadir & 181916064524200 \\
\hline $15-42$ & 17 & M. Griffin & Nadir & 181916064524300 \\
\hline $15-43$ & 17 & J. Sanchez & Nadir & 181917064524501 \\
\hline $15-44$ & 17 & $\begin{array}{l}\text { VI Government/ } \\
\text { Race track }\end{array}$ & Nadir & 181917064524500 \\
\hline $15-45$ & 17 & $\begin{array}{l}\text { VI Government/ } \\
\text { Abattoir west well }\end{array}$ & Nadir & 181918064524400 \\
\hline $16-1$ & 18 & Harrigan & Frydenhoj & 181933064515300 \\
\hline $16-2$ & 18 & Peters & Frydenhoj & 181932064515600 \\
\hline $16-3$ & 18 & E. Haynes & Frydenhoj & 181929064515600 \\
\hline $16-4$ & 18 & $\begin{array}{l}\text { VI Government/ } \\
\text { near A. Richards }\end{array}$ & Frydenhoj & 181929064515700 \\
\hline $16-5$ & 18 & A. Richards & Frydenhoj & 181929064515900 \\
\hline $16-6$ & 18 & J. Brin & Frydenhoj & 181929064520000 \\
\hline $16-7$ & 18 & J. Gimenez & Frydenhoj & 181927064520000 \\
\hline $16-8$ & 18 & Compass Point & Frydenhoj & 181913064515800 \\
\hline $16-9$ & 18 & D. Scott & Frydenhoj & 181914064515100 \\
\hline $16-10$ & 18 & L. Olive & Nazareth & 181924064512300 \\
\hline $16-11$ & 18 & Eudora Kean School & Nazareth & 181936064511900 \\
\hline $16-12$ & 18 & $\begin{array}{l}\text { American Yacht } \\
\text { Harbor }\end{array}$ & Nazareth & 181939064510700 \\
\hline $16-13$ & 18 & Red Hook Plaza & Smith Bay & 181943064510800 \\
\hline
\end{tabular}


Appendix A. Well names, Estates, and site-identification numbers of wells on St. Thomas, U.S. Virgin Islands--Continued

\begin{tabular}{|c|c|c|c|c|}
\hline $\begin{array}{l}\text { Wel1 } \\
\text { number }\end{array}$ & $\begin{array}{l}\text { Figure } \\
\text { number }\end{array}$ & Well name & $\begin{array}{c}\text { Estate } \\
\text { (see figure 2) }\end{array}$ & $\begin{array}{c}\text { Site } \\
\text { identification } \\
\text { number }\end{array}$ \\
\hline $16-14$ & 18 & $\begin{array}{l}\text { Cabrita Point } \\
\text { Condo }\end{array}$ & Nazareth & 181939064502500 \\
\hline $16-15$ & 18 & s. Harrington & Nazareth & 181921064505900 \\
\hline $16-16$ & 18 & R. Alexander & Nazareth & 181920064505800 \\
\hline $16-17$ & 18 & G. Brown & Nazareth & 181919064505700 \\
\hline $16-18$ & 18 & Cowpet East & Nazareth & 181913064504000 \\
\hline $16-19$ & 18 & $\begin{array}{l}\text { Elysian Beach } \\
\text { Resort }\end{array}$ & Nazareth & 181911064504300 \\
\hline $16-20$ & 18 & Cowpet West 2 & Nazareth & 181910064504300 \\
\hline $16-21$ & 18 & Cowpet West 1 & Nazareth & 181905064504700 \\
\hline $16-22$ & 18 & $\begin{array}{c}\text { Anchorage Beach } \\
\text { Villas Condo } 1\end{array}$ & Nazareth & 181904064505100 \\
\hline $16-23$ & 18 & $\begin{array}{l}\text { St. Thomas } \\
\text { Yacht Club }\end{array}$ & Nazareth & 181903064505000 \\
\hline $16-24$ & 18 & $\begin{array}{c}\text { Anchorage Beach } \\
\text { Villa Condo } 2\end{array}$ & Nazareth & 181900064504600 \\
\hline
\end{tabular}


Appendix B. Lithologic descriptions and remarks for wells on St. Thomas, U.S. Virgin Islands

$[--$, indicates data not available $]$

$\begin{array}{ccc}\text { Well } & \text { Figure } & \text { Lithologic description } \\ \text { number } & \text { and depth interval } & \text { Remarks } \\ & & \text { (feet) }\end{array}$

$2-1$
$3-1$
$3-2$
$3-3$
$3-4$
$3-5$
$3-6$
$3-7$

Unconsolidated material 0-18 Bedrock 18

5

5

Unconsolidated material 0-45 Bedrock 45

5

5

5

5

$3-8$
$3-9$

$3-10$

$3-11$

$3-12$

$3-13$

$3-14$

$3-15$

$3-16$

5

5

5

5

5

5

5

6- 1

8

$7-1 \quad 9$

$7-2 \quad 9$

$7-3 \quad 9$

$7-4 \quad 9$

$7-5 \quad 9$

$7-6 \quad 9$
Unconsolidated material 0-30 Bedrock 30

Unconsolidated material 0-26 Bedrock 26

Unconsolidated material 0-55 Bedrock 55

Unconsolidated material $0-30$ Bedrock 30

Unconsolidated material $0-12$ Bedrock 12
Owner estimated daily pumpage is 1,300 gallons.

Well is plugged at 25 feet.

Water-level data for 1963-65 available under "Wel1 4" in Robison and others, 1973.

Well used in supply of condominiums. Well owned by $D$ and $C$ Development.

Owner estimated daily pumpage is 10,000 gallons. Well could pump 40,000 gallons per day. Well used in supply of condominiums. Well owned by $D$ and $C$ Development.

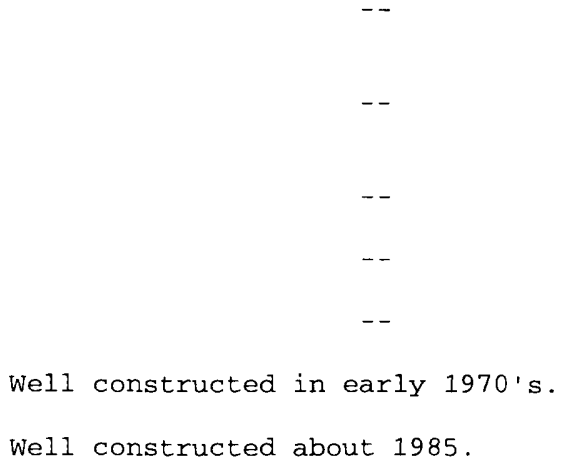

Well dug in 1964 or 1965 . Owner estimated daily pumpage is 5,000 gallons.

Well dug about 1750 .

Maintenance personel estimated daily pumpage is 20,000 gallons. 
Appendix B. Lithologic descriptions and remarks for wells on St. Thomas, U.S. Virgin Islands--Continued [--, indicates data not available]

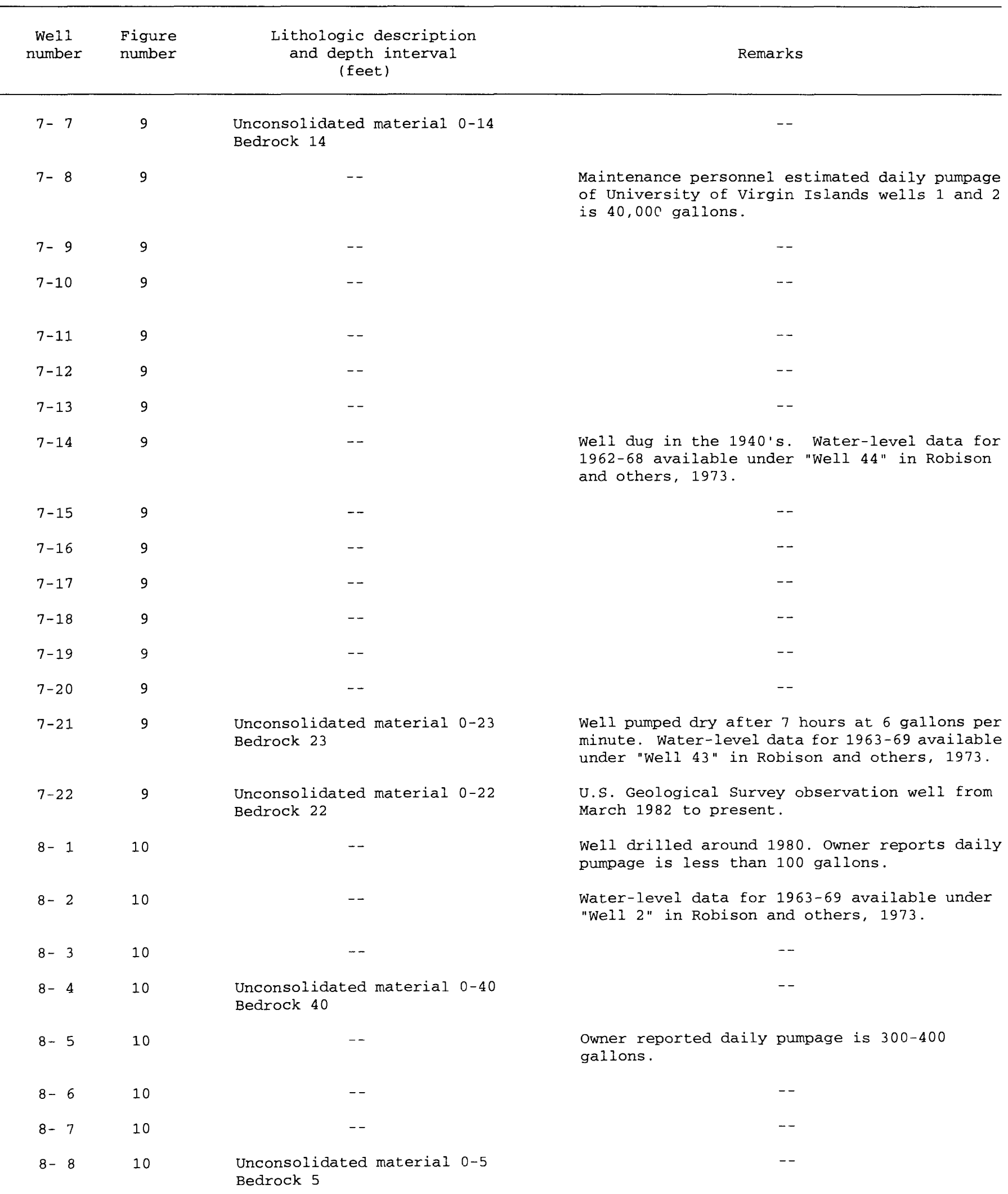


Appendix B. Lithologic descriptions and remarks for wells on St. Thomas, U.S. Virgin Islands--Continued [--, indicates data not available]

\begin{tabular}{|c|c|c|c|}
\hline $\begin{array}{l}\text { Well } \\
\text { number }\end{array}$ & $\begin{array}{l}\text { Figure } \\
\text { number }\end{array}$ & $\begin{array}{l}\text { Lithologic description } \\
\text { and depth interval } \\
\text { (feet) }\end{array}$ & Remarks \\
\hline $8-9$ & 10 & - & Owner reports weekly pumpage of 170 gallons. \\
\hline $8-10$ & 10 & - & $\begin{array}{l}\text { Owner reported yield of } 5-15 \text { gallons per minute } \\
\text { in dry season and } 10-15 \text { gallons per minute in } \\
\text { the wet season. }\end{array}$ \\
\hline $8-11$ & 10 & $\begin{array}{l}\text { Unconsolidated material } 0-26 \\
\text { Bedrock } 26\end{array}$ & -- \\
\hline $8-12$ & 10 & -- & $\begin{array}{l}\text { Well drilled in the late } 1970^{\prime} \mathrm{s} \text {. Owner } \\
\text { estimated daily pumpage is } 80-120 \text { gallons. }\end{array}$ \\
\hline $8-13$ & 10 & $\begin{array}{l}\text { Unconsolidated material } 0-14 \\
\text { Bedrock } 14\end{array}$ & -- \\
\hline $8-14$ & 10 & $\begin{array}{l}\text { Unconsolidated material } 0-34 \\
\text { Bedrock } 34\end{array}$ & -- \\
\hline $8-15$ & 10 & -- & Well drilled in $1980^{\prime} \mathrm{s}$. \\
\hline $8-16$ & 10 & - & Well drilled early 1980's. \\
\hline $8-17$ & 10 & -- & -- \\
\hline $8-18$ & 10 & $\begin{array}{l}\text { Unconsolidated material } 0-10 \\
\text { Bedrock } 10\end{array}$ & Driller reported well as dry. \\
\hline $8-19$ & 10 & - & Well blocked at 25 feet. No water level. \\
\hline $8-20$ & 10 & $\begin{array}{l}\text { Unconsolidated material } 0-40 \\
\text { Bedrock } 40\end{array}$ & -- \\
\hline $8-21$ & 10 & -- & Well was dug about 1955. Well is capped. \\
\hline $8-22$ & 10 & -- & -- \\
\hline $8-23$ & 10 & -- & Well was dug before the 1900 's. \\
\hline $8-24$ & 10 & -- & -- \\
\hline $8-25$ & 10 & $\begin{array}{l}\text { Unconsolidated material } 0-4 \\
\text { Bedrock } 4\end{array}$ & -- \\
\hline $8-26$ & 10 & $\begin{array}{l}\text { Unconsolidated material } 0-13 \\
\text { Bedrock } 13\end{array}$ & -- \\
\hline $8-27$ & 10 & -- & $\begin{array}{l}\text { Well was cleaned out and new } 4 \text {-inch PVC pipe } \\
\text { installed in } 1983 .\end{array}$ \\
\hline $8-28$ & 10 & -- & -- \\
\hline $8-29$ & 10 & $\begin{array}{l}\text { Unconsolidated material } 0-24 \\
\text { Bedrock } 24\end{array}$ & $\begin{array}{l}\text { Pumping water level is } 60-62 \text { feet. Well used } \\
\text { in supply of apartments. }\end{array}$ \\
\hline $8-30$ & 10 & -- & Depth to water is approximately 31 feet. \\
\hline $8-31$ & 10 & -- & -- \\
\hline $8-32$ & 10 & -- & -- \\
\hline $8-33$ & 10 & -- & -- \\
\hline
\end{tabular}


Appendix B. Lithologic descriptions and remarks for wells on St. Thomas, U.S. Virgin Islands--Continued $[--$ indicates data not available]

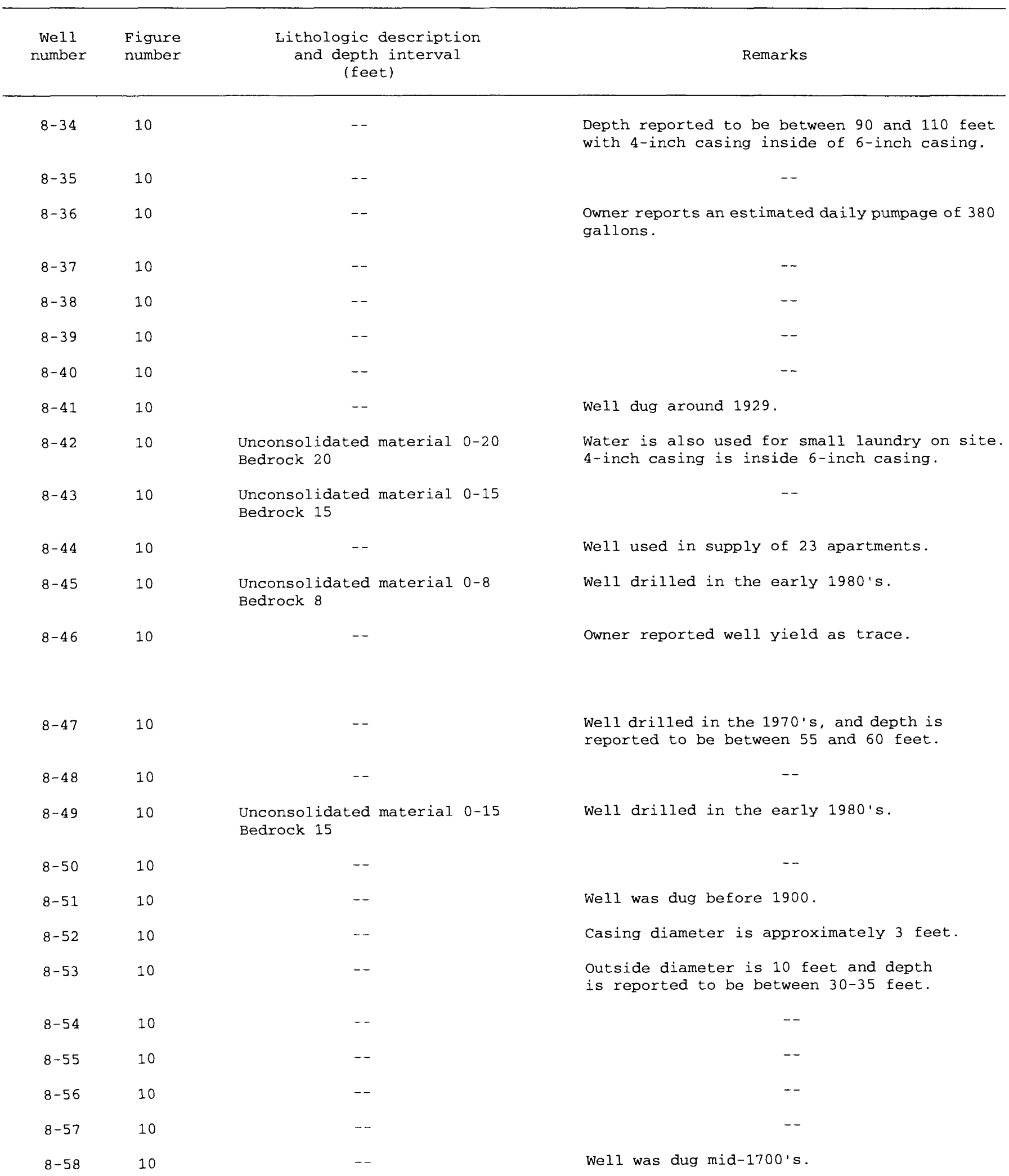


Appendix B. Lithologic descriptions and remarks for wells on St. Thomas, U.S. Virgin Islands--Continued

\section{[--, indicates data not available]}

\begin{tabular}{|c|c|c|c|}
\hline $\begin{array}{l}\text { Well } \\
\text { number }\end{array}$ & $\begin{array}{l}\text { Figure } \\
\text { number }\end{array}$ & $\begin{array}{c}\text { Lithologic description } \\
\text { and depth interval } \\
\text { (feet) }\end{array}$ & Remarks \\
\hline $8-59$ & 10 & $\begin{array}{l}\text { Unconsolidated material } 0-28 \\
\text { Bedrock } 28\end{array}$ & $\begin{array}{l}\text { Well used in supply of aproximately } 13 \\
\text { apartments. }\end{array}$ \\
\hline $8-60$ & 10 & -- & -- \\
\hline $8-61$ & 10 & $\begin{array}{l}\text { Unconsolidated material } 0-40 \\
\text { Bedrock } 40\end{array}$ & $\begin{array}{l}\text { Owner estimated daily pumpage is } 14,000 \\
\text { gallons. }\end{array}$ \\
\hline $8-62$ & 10 & -- & -- \\
\hline $8-63$ & 10 & -- & $\begin{array}{l}\text { Owner estimated daily pumpage is } 250-400 \\
\text { gallons. }\end{array}$ \\
\hline $8-64$ & 10 & -- & -- \\
\hline $8-65$ & 10 & Unconsolidated material $0-52$ & -- \\
\hline $9-1$ & 11 & -- & 24-inch casing inside of the 120-inch casing. \\
\hline $9-2$ & 11 & Unconsolidated material $0-65$ & -- \\
\hline $9-3$ & 11 & -- & -- \\
\hline $9-4$ & 11 & -- & Well dug about 1750 . \\
\hline $9-5$ & 11 & $\begin{array}{l}\text { Unconsolidated material } 0-5 \\
\text { Bedrock } 5\end{array}$ & -- \\
\hline $9-6$ & 11 & $\begin{array}{l}\text { Unconsolidated material 0-5 } \\
\text { Bedrock } 5\end{array}$ & $\begin{array}{l}\text { Maintenance personnel estimated daily pumpage } \\
\text { is } 1,500 \text { gallons or } 2,000 \text { gallons if rainfall } \\
\text { is high. }\end{array}$ \\
\hline $9-7$ & 11 & $\begin{array}{l}\text { Unconsolidated material } 0-3 \\
\text { Bedrock } 3\end{array}$ & -- \\
\hline $9-8$ & 11 & $\begin{array}{l}\text { Unconsolidated material } 0-10 \\
\text { Bedrock } 10\end{array}$ & -- \\
\hline $9-9$ & 11 & -- & 4-inch casing inside of 6 -inch casing. \\
\hline $9-10$ & 11 & -- & Well drilled before 1975 . \\
\hline $9-11$ & 11 & -- & $\begin{array}{l}\text { 4-inch peforated casing inside of the } 6 \text {-inch } \\
\text { casing. }\end{array}$ \\
\hline $9-12$ & 11 & -- & -- \\
\hline $9-13$ & 11 & -- & -- \\
\hline $9-14$ & 11 & -- & $\begin{array}{l}\text { Water is also used for golf course. Well used } \\
\text { in supply of condominiums. Owned by M.R. } \\
\text { Holdings Inc. }\end{array}$ \\
\hline $9-15$ & 11 & -- & $\begin{array}{l}\text { Water is also used for golf course. Well used } \\
\text { in supply of condominiums. Owned by M.R. } \\
\text { Holdings Inc. }\end{array}$ \\
\hline $9-16$ & 11 & -- & -- \\
\hline $9-17$ & 11 & -- & -- \\
\hline
\end{tabular}


Appendix B. Lithologic descriptions and remarks for wells on St. Thomas, U.S. Virgin Islands--Continued [--, indicates data not available]

\begin{tabular}{|c|c|c|c|}
\hline $\begin{array}{l}\text { Wel1 } \\
\text { number }\end{array}$ & $\begin{array}{l}\text { Figure } \\
\text { number }\end{array}$ & $\begin{array}{l}\text { Lithologic description } \\
\text { and depth interval } \\
\text { (feet) }\end{array}$ & Remarks \\
\hline $9-18$ & 11 & -- & -- \\
\hline $9-19$ & 11 & -- & $\begin{array}{l}\text { Water is also used for golf course. Well used } \\
\text { in supply of condominiums. Owmed by M.R. } \\
\text { Holdings Inc. }\end{array}$ \\
\hline $9-20$ & 11 & -- & -- \\
\hline $9-21$ & 11 & -- & $\begin{array}{l}\text { Estimated pumping water level is } 75 \text { feet. Well } \\
\text { used in supply of condominiums. Owned by M.R. } \\
\text { Holdings Inc. }\end{array}$ \\
\hline $9-22$ & 11 & -- & Well owned by M.R. Holdings Inc. \\
\hline $9-23$ & 11 & -- & Well owned by M.R. Holdings Inc. \\
\hline $9-24$ & 11 & -- & $\begin{array}{l}\text { Wel1 dug before } 1860 \text {. Water-level data for } \\
1962-68 \text { available under "Wel1 } 8 \text { " in Robison and } \\
\text { others } 1973 \text {. }\end{array}$ \\
\hline $9-25$ & 11 & -- & Well owned by M.R. Holdings Inc. \\
\hline $9-26$ & 11 & -- & $\begin{array}{l}\text { Water-level data for } 1963-69 \text { available under } \\
\text { "Well } 7 \text { " in Robison and others, } 1973 .\end{array}$ \\
\hline $9-27$ & 11 & - & -- \\
\hline $9-28$ & 11 & -- & -- \\
\hline $9-29$ & 11 & -- & Well owned by M.R. Holdings Inc. \\
\hline $9-30$ & 11 & -- & -- \\
\hline $9-31$ & 11 & -- & - \\
\hline $9-32$ & 11 & -- & Well partially blocked at 17 feet. \\
\hline $9-33$ & 11 & $\begin{array}{l}\text { Unconsolidated material } 0-10 \\
\text { Bedrock } 10\end{array}$ & $\begin{array}{l}\text { 4-inch casing reported from } 0 \text { to } 20 \text { feet. } \\
\text { Estimated daily pumpage is } 450 \text { gallons. }\end{array}$ \\
\hline $9-34$ & 11 & -- & $\begin{array}{l}\text { Well dug during the } 1700 \text { 's. Water-level data } \\
\text { data for } 1962-64 \text { available under "Well } 5 \text { " in } \\
\text { Robison and others, } 1973 \text {. }\end{array}$ \\
\hline $9-35$ & 11 & -- & -- \\
\hline $9-36$ & 11 & -- & -- \\
\hline $9-37$ & 11 & -- & -- \\
\hline $9-38$ & 11 & -- & 7.5-inch casing visible at surface. \\
\hline $9-39$ & 11 & -- & $\begin{array}{l}\text { Well is blocked at } 70 \text { feet, no water level. } \\
\text { Water-level data for } 1963-69 \text { available under } \\
\text { "Well 6" in Robison and others, } 1973 \text {. }\end{array}$ \\
\hline $9-40$ & 11 & -- & -- \\
\hline $9-41$ & 11 & -- & -- \\
\hline $9-42$ & 11 & -- & -- \\
\hline
\end{tabular}


Appendix B. Lithologic descriptions and remarks for wells on St. Thomas, U.S. Virgin Islands--Continued [--, indicates data not available]

\begin{tabular}{|c|c|c|c|}
\hline $\begin{array}{l}\text { Well } \\
\text { number }\end{array}$ & $\begin{array}{l}\text { Figure } \\
\text { number }\end{array}$ & $\begin{array}{l}\text { Lithologic description } \\
\text { and depth interval } \\
\text { (feet) }\end{array}$ & Remarks \\
\hline $9-43$ & 11 & -- & We11 dug in the mid-1700's. \\
\hline $9-44$ & 11 & -- & -- \\
\hline $9-45$ & 11 & -- & -- \\
\hline $9-46$ & 11 & -- & Well dug before 1919. \\
\hline $9-47$ & 11 & -- & -- \\
\hline $9-48$ & 11 & -- & Owner plans to use well for public supply. \\
\hline $9-49$ & 11 & $\begin{array}{l}\text { Unconsolidated material } 0-15 \\
\text { Bedrock } 15\end{array}$ & -- \\
\hline $9-50$ & 11 & -- & -- \\
\hline $9-51$ & 11 & $\begin{array}{l}\text { Unconsolidated material } 0-6 \\
\text { Bedrock } 6\end{array}$ & -- \\
\hline $9-52$ & 11 & $\begin{array}{l}\text { Unconsolidated material } 0-40 \\
\text { Bedrock } 40\end{array}$ & $\begin{array}{l}\text { 6-inch casing from } 0 \text { to } 100 \text { feet with } 4 \text {-inch } \\
\text { slotted screen inside. }\end{array}$ \\
\hline $9-53$ & 11 & $\begin{array}{l}\text { Unconsolidated material } 0-25 \\
\text { Bedrock } 25\end{array}$ & 4 -inch casing inside of the 6 -inch casing. \\
\hline $9-54$ & 11 & -- & -- \\
\hline $9-55$ & 11 & -- & Well dug about 1945 \\
\hline $9-56$ & 11 & -- & $\begin{array}{l}\text { Well dug in late } 1950 \text { 's and casing diameter is } \\
48-60 \text { inches. }\end{array}$ \\
\hline $9-57$ & 11 & Unconsolidated material $0-36$ & Well owned by Burnette Tower Apartments. \\
\hline $9-58$ & 11 & -- & Well dug in the $1950^{\prime} \mathrm{s}$. \\
\hline $9-59$ & 11 & -- & Drilled in late 1980 's. \\
\hline $9-60$ & 11 & -- & Well is blocked at 31 feet. \\
\hline $9-61$ & 11 & -- & $\begin{array}{l}\text { U.S. Geological Survey observation well. Well } \\
\text { owned by Water and Power Authority. }\end{array}$ \\
\hline $9-62$ & 11 & -- & Well owned by the Water And Power Authority. \\
\hline $9-63$ & 11 & $\begin{array}{l}\text { Unconsolidated material } 0-45 \\
\text { Bedrock } 45\end{array}$ & Well owned by the water And Power Authority. \\
\hline $9-64$ & 11 & -- & $\begin{array}{l}\text { Well was reported as dry, yield less than } 1 \\
\text { gallon per minute. }\end{array}$ \\
\hline $9-65$ & 11 & $\begin{array}{l}\text { Unconsolidated material } 0-31 \\
\text { Bedrock } 31\end{array}$ & -- \\
\hline $9-66$ & 11 & -- & Well owned by the water and Power Authority. \\
\hline $9-67$ & 11 & $\begin{array}{l}\text { Unconsolidated material } 0-60 \\
\text { Bedrock } 60\end{array}$ & 6 -inch casing from 0 to 60 feet. \\
\hline $9-68$ & 11 & $\begin{array}{l}\text { Unconsolidated material } 0-45 \\
\text { Bedrock } 45\end{array}$ & -- \\
\hline
\end{tabular}


Appendix B. Lithologic descriptions and remarks for wells on St. Thomas, U.S. Virgin Islands--Continued [--, indicates data not available]

\begin{tabular}{|c|c|c|c|}
\hline $\begin{array}{l}\text { Well } \\
\text { number }\end{array}$ & $\begin{array}{l}\text { Figure } \\
\text { number }\end{array}$ & $\begin{array}{l}\text { Lithologic description } \\
\text { and depth interval } \\
\text { (feet) }\end{array}$ & Remarks \\
\hline $9-69$ & 11 & Unconsolidated material $0-67$ & -- \\
\hline $9-70$ & 11 & $\begin{array}{l}\text { Unconsolidated material } 0-60 \\
\text { Bedrock } 60\end{array}$ & -- \\
\hline $9-71$ & 11 & $\begin{array}{l}\text { Unconsolidated material } 0-45 \\
\text { Bedrock } 45\end{array}$ & -- \\
\hline $9-72$ & 11 & -- & Well is a 36 -inch by 108 -inch rectangle. \\
\hline $9-73$ & 11 & -- & -- \\
\hline $9-74$ & 11 & Unconsolidated material $0-56$ & -- \\
\hline $9-75$ & 11 & -- & Well was dug in the early 1960 's. \\
\hline $9-76$ & 11 & -- & 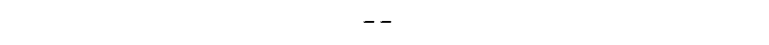 \\
\hline $9-77$ & 11 & -- & -- \\
\hline $9-78$ & 11 & -- & -- \\
\hline $9-79$ & 11 & -- & Well constructed about 40 years ago. \\
\hline $9-80$ & 11 & -- & $\begin{array}{l}\text { Well owned by Wallace Turnball and used in } \\
\text { supply of apartments. }\end{array}$ \\
\hline $9-81$ & 11 & -- & $\begin{array}{l}\text { Well owned by Wallace Turnball and used in } \\
\text { supply of approximately } 15 \text { apartments. }\end{array}$ \\
\hline $9-82$ & 11 & $\begin{array}{l}\text { Unconsolidated material } 0-60 \\
\text { Bedrock } 60\end{array}$ & -- \\
\hline $9-83$ & 11 & -- & $\begin{array}{l}\text { Well may be blocked at } 38 \text { feet. } 4 \text {-inch casing } \\
\text { inside of } 6 \text {-inch casing. }\end{array}$ \\
\hline $9-84$ & 11 & -- & -- \\
\hline $9-85$ & 11 & -- & -- \\
\hline $9-86$ & 11 & -- & -- \\
\hline $9-87$ & 11 & -- & -- \\
\hline $9-88$ & 11 & $\begin{array}{l}\text { Unconsolidated material } 0-56 \\
\text { Bedrock } 56\end{array}$ & -- \\
\hline $9-89$ & 11 & $\begin{array}{l}\text { Unconsolidated material } 0-45 \\
\text { Bedrock } 45\end{array}$ & -- \\
\hline $9-90$ & 11 & -- & -- \\
\hline $9-91$ & 11 & -- & -- \\
\hline $9-92$ & 11 & -- & $\begin{array}{l}\text { Well drilled about } 1986 \text {. Well owned by Thomas } \\
\text { Frett and used in supply of } 5 \text { apartments. }\end{array}$ \\
\hline $9-93$ & 11 & $\begin{array}{l}\text { Unconsolidated material } 0-16 \\
\text { Bedrock } 16\end{array}$ & -- \\
\hline
\end{tabular}


Appendix B. Lithologic descriptions and remarks for wells on St. Thomas, U.S. Virgin Islands--Continued $[-$, indicates data not available $]$

\begin{tabular}{|c|c|c|c|}
\hline $\begin{array}{l}\text { We11 } \\
\text { number }\end{array}$ & $\begin{array}{l}\text { Figure } \\
\text { number }\end{array}$ & $\begin{array}{l}\text { Lithologic description } \\
\text { and depth interval } \\
\text { (feet) }\end{array}$ & Remarks \\
\hline $9-94$ & 11 & $\begin{array}{l}\text { Unconsolidated material } 0-2 \\
\text { Bedrock } 2\end{array}$ & Owner estimated daily pumpage is 1,500 gallons. \\
\hline $9-95$ & 11 & -- & $\begin{array}{l}\text { Well was dug before } 1900 \text { and is oval with } \\
20 \text {-foot diameter at widest point. Water-level } \\
\text { data for } 1962-64 \text { available under "well 16" in } \\
\text { Robison and others, } 1973 \text {. }\end{array}$ \\
\hline $9-96$ & 11 & -- & Well was dug during the 1700 's. \\
\hline $9-97$ & 11 & $\begin{array}{l}\text { Unconsolidated material } 0-45 \\
\text { Bedrock } 45\end{array}$ & -- \\
\hline $9-98$ & 11 & $\begin{array}{l}\text { Unconsolidated material } 0-35 \\
\text { Bedrock } 35\end{array}$ & -- \\
\hline $9-99$ & 11 & $\begin{array}{l}\text { Unconsolidated material } 0-40 \\
\text { Bedrock } 40\end{array}$ & -- \\
\hline $10-1$ & 12 & -- & -- \\
\hline $10-2$ & 12 & -- & -- \\
\hline $10-3$ & 12 & Unconsolidated material $0-45$ & -- \\
\hline $10-4$ & 12 & -- & -- \\
\hline $10-5$ & 12 & -- & -- \\
\hline $10-6$ & 12 & -- & -- \\
\hline $10-7$ & 12 & -- & -- \\
\hline $10-8$ & 12 & - & -- \\
\hline $10-9$ & 12 & -- & U.S. Geological Survey observation well. \\
\hline $10-10$ & 12 & $\begin{array}{l}\text { Unconsolidated material } 0-30 \\
\text { Bedrock } 30\end{array}$ & $\begin{array}{l}\text { Owner estimated daily pumpage is } 25,000 \\
\text { gallons. }\end{array}$ \\
\hline $10-11$ & 12 & $\begin{array}{l}\text { Unconsolidated material } 0-20 \\
\text { Bedrock } 20\end{array}$ & -- \\
\hline $10-12$ & 12 & $\begin{array}{l}\text { Unconsolidated material } 0-28 \\
\text { Bedrock } 289\end{array}$ & $\begin{array}{l}\text { Well reported dry. Owner estimated daily } \\
\text { pumpage is } 25,000 \text { gallons. }\end{array}$ \\
\hline $10-13$ & 12 & $\begin{array}{l}\text { Unconsolidated material } 0-40 \\
\text { Bedrock } 40\end{array}$ & -- \\
\hline $10-14$ & 12 & $\begin{array}{l}\text { Unconsolidated material } 0-30 \\
\text { Bedrock } 30\end{array}$ & -- \\
\hline $10-15$ & 12 & -- & $\begin{array}{l}\text { Property manager reported well could yield } \\
38,000 \text { gallons per day. }\end{array}$ \\
\hline $10-16$ & 12 & -- & Yield was reported bailed. \\
\hline $10-17$ & 12 & -- & Inside diameter 85-95 inches. \\
\hline
\end{tabular}


Appendix B. Lithologic descriptions and remarks for wells on St. Thomas, U.S. Virgin Islands--Continued [--, indicates data not available]

\begin{tabular}{|c|c|c|c|}
\hline $\begin{array}{l}\text { Well } \\
\text { number }\end{array}$ & $\begin{array}{l}\text { Figure } \\
\text { number }\end{array}$ & $\begin{array}{l}\text { Lithologic description } \\
\text { and depth interval } \\
\text { (feet) }\end{array}$ & Remarks \\
\hline $10-18$ & 12 & $\begin{array}{l}\text { Unconsolidated material } 0-10 \\
\text { Bedrock } 10\end{array}$ & $\begin{array}{l}\text { Well drilled in the } 1960 \text { 's. Reported yield is } \\
\text { bailed. }\end{array}$ \\
\hline $10-19$ & 12 & -- & Well dug around 1938. \\
\hline $10-20$ & 12 & -- & $\begin{array}{l}\text { 60-72 inch diameter well dug before } 1943 \text {. } \\
\text { Water-level data for } 1967-69 \text { available under } \\
\text { "Well 18" in Robison and others, } 1973 \text {. }\end{array}$ \\
\hline $10-21$ & 12 & -- & -- \\
\hline $10-22$ & 12 & -- & -- \\
\hline $10-23$ & 12 & -- & -- \\
\hline $10-24$ & 12 & -- & -- \\
\hline $10-25$ & 12 & -- & Well drilled in 1972 or 1973. \\
\hline $10-26$ & 12 & -- & Well drilled in 1972 or 1973. \\
\hline $10-27$ & 12 & $\begin{array}{l}\text { Unconsolidated material } 0-20 \\
\text { Bedrock } 20\end{array}$ & -- \\
\hline $10-28$ & 12 & -- & $\begin{array}{l}\text { Well covered over. Water-level data for } \\
1958-69 \text { available under "Well } 26 " \text { in Robison } \\
\text { and other, } 1973 \text {. }\end{array}$ \\
\hline $10-29$ & 12 & -- & -- \\
\hline $10-30$ & 12 & -- & -- \\
\hline $10-31$ & 12 & -- & -- \\
\hline $10-32$ & 12 & -- & Well drilled in the 1960 's. \\
\hline $10-33$ & 12 & -- & -- \\
\hline $10-34$ & 12 & -- & Well caved in to 105 feet. \\
\hline $10-35$ & 12 & -- & -- \\
\hline $10-36$ & 12 & $\begin{array}{l}\text { Unconsolidated material } 0-30 \\
\text { Bedrock } 30\end{array}$ & -- \\
\hline $10-37$ & 12 & -- & $\begin{array}{l}\text { Owner reported depth } 125 \text { feet, previous } \\
\text { inventory reported depth of } 210 \text { feet. }\end{array}$ \\
\hline $10-38$ & 12 & -- & $\begin{array}{l}\text { Well reported to be redrilled to } 225 \text { feet in } \\
1979 \text {. Water-level data for } 1967-69 \text { available } \\
\text { under "Well } 24 \text { " in Robison and others, } 1973 \text {. }\end{array}$ \\
\hline $10-39$ & 12 & -- & -- \\
\hline $10-40$ & 12 & -- & $\begin{array}{l}\text { Owner reported depth to be } 385 \text { feet. Previous } \\
\text { Previous inventory reported depth of } 225 \text { feet } \\
\text { with casing to } 40 \text { feet. }\end{array}$ \\
\hline $10-41$ & 12 & - & -- \\
\hline
\end{tabular}


Appendix B. Lithologic descriptions and remarks for wells on St. Thomas, U.S. Virgin Islands--Continued [--, indicates data not available]

\begin{tabular}{|c|c|c|c|}
\hline $\begin{array}{l}\text { We11 } \\
\text { number }\end{array}$ & $\begin{array}{l}\text { Figure } \\
\text { number }\end{array}$ & $\begin{array}{l}\text { Lithologic description } \\
\text { and depth interval } \\
\text { (feet) }\end{array}$ & Remarks \\
\hline $10-42$ & 12 & -- & $\begin{array}{l}\text { Wel1 dug before } 1942 \text {. Water-level data for } \\
1962-63 \text { available under "Wel1 } 23^{\prime \prime} \text { in Robison } \\
\text { and others, } 1973 \text {. }\end{array}$ \\
\hline $10-43$ & 12 & -- & $\begin{array}{l}\text { Water is being used at temporary archeologic } \\
\text { site. Water-1evel data for } 1965-69 \text { available } \\
\text { under "We11 19" in Robison and others, } 1973 \text {. }\end{array}$ \\
\hline $10-44$ & 12 & -- & Well is covered over. \\
\hline $10-45$ & 12 & $\begin{array}{l}\text { Unconsolidated material } 0-30 \\
\text { Bedrock } 30\end{array}$ & -- \\
\hline $10-46$ & 12 & $\begin{array}{l}\text { Unconsolidated material } 0-24 \\
\text { Bedrock } 24\end{array}$ & -- \\
\hline $10-47$ & 12 & -- & -- \\
\hline $10-48$ & 12 & $\begin{array}{l}\text { Unconsolidated material } 0-10 \\
\text { Bedrock } 10\end{array}$ & -- \\
\hline $10-49$ & 12 & $\begin{array}{l}\text { Unconsolidated material } 0-32 \\
\text { Bedrock } 32\end{array}$ & $\begin{array}{l}\text { Well is owned by the U.S. Virgin Islands } \\
\text { Housing Authority. }\end{array}$ \\
\hline $10-50$ & 12 & $\begin{array}{l}\text { Unconsolidated material } 0-32 \\
\text { Bedrock } 32\end{array}$ & -- \\
\hline $10-51$ & 12 & -- & $\begin{array}{l}\text { Well is owned by the U.S. Virgin Islands } \\
\text { Housing Authority. }\end{array}$ \\
\hline $10-52$ & 12 & -- & -- \\
\hline $10-53$ & 12 & $\begin{array}{l}\text { Unconsolidated material } 0-12 \\
\text { Bedrock } 12\end{array}$ & $\begin{array}{l}\text { Water-level data for } 1963-69 \text { available under } \\
\text { "We11 9" in Robison and others, } 1973 .\end{array}$ \\
\hline $10-54$ & 12 & $\begin{array}{l}\text { Unconsolidated material } 0-18 \\
\text { Bedrock } 18\end{array}$ & -- \\
\hline $10-55$ & 12 & -- & -- \\
\hline $10-56$ & 12 & -- & -- \\
\hline $10-57$ & 12 & -- & -- \\
\hline $10-58$ & 12 & $\begin{array}{l}\text { Unconsolidated material } 0-65 \\
\text { Bedrock } 65\end{array}$ & well screen diameter is 5 inches. \\
\hline $10-59$ & 12 & -- & -- \\
\hline $10-60$ & 12 & $\begin{array}{l}\text { Unconsolidated material } 0-28 \\
\text { Bedrock } 28\end{array}$ & -- \\
\hline $10-61$ & 12 & $\begin{array}{l}\text { Unconsolidated material } 0-30 \\
\text { Bedrock } 30\end{array}$ & -- \\
\hline $10-62$ & 12 & -- & well drilled in the early 1970 's. \\
\hline $10-63$ & 12 & $\begin{array}{l}\text { Unconsolidated material } 0-80 \\
\text { Bedrock } 80\end{array}$ & -- \\
\hline
\end{tabular}


Appendix B. Lithologic descriptions and remarks for wells on St. Thomas, U.S. Virgin Islands--Continued [--, indicates data not available]

\begin{tabular}{|c|c|c|c|}
\hline $\begin{array}{l}\text { Well } \\
\text { number }\end{array}$ & $\begin{array}{l}\text { Figure } \\
\text { number }\end{array}$ & $\begin{array}{l}\text { Lithologic description } \\
\text { and depth interval } \\
\text { (feet) }\end{array}$ & Remarks \\
\hline $10-64$ & 12 & -- & -- \\
\hline $10-65$ & 12 & -- & -- \\
\hline $10-66$ & 12 & -- & Well drilled in 1963 or 1964 . \\
\hline $10-67$ & 12 & -- & -- \\
\hline $10-68$ & 12 & -- & -- \\
\hline $10-69$ & 12 & -- & -- \\
\hline $10-70$ & 12 & -- & -- \\
\hline $10-71$ & 12 & -- & -- \\
\hline $10-72$ & 12 & -- & -- \\
\hline $10-73$ & 12 & -- & -- \\
\hline $10-74$ & 12 & -- & -- \\
\hline $11-1$ & 13 & $\begin{array}{l}\text { Unconsolidated material } 0-3 \\
\text { Bedrock } 3\end{array}$ & 8-inch casing at surface. \\
\hline $11-2$ & 13 & -- & -- \\
\hline $11-3$ & 13 & -- & $\begin{array}{l}\text { Well is square } 96 \text { by } 96 \text { inches. Water-level } \\
\text { data for } 1962-64 \text { available under "Well 11" in } \\
\text { Robison and others, } 1973 \text {. }\end{array}$ \\
\hline $11-4$ & 13 & $\begin{array}{l}\text { Unconsolidated material } 0-40 \\
\text { Bedrock } 40\end{array}$ & -- \\
\hline $11-5$ & 13 & -- & -- \\
\hline $11-6$ & 13 & -- & -- \\
\hline $11-7$ & 13 & -- & $\begin{array}{l}\text { Water-level data for } 1965-69 \text { available under } \\
\text { "Well 12" in Robison and others, } 1973 \text {. }\end{array}$ \\
\hline $14-1$ & 16 & -- & Well reported caved in to 508 feet. \\
\hline $14-2$ & 16 & $\begin{array}{l}\text { Unconsolidated material } 0-28 \\
\text { Bedrock } 28\end{array}$ & -- \\
\hline $14-3$ & 16 & $\begin{array}{l}\text { Unconsolidated material } 0-54 \\
\text { Bedrock } 54\end{array}$ & -- \\
\hline $14-4$ & 16 & $\begin{array}{l}\text { Unconsolidated material } 0-10 \\
\text { Bedrock } 10\end{array}$ & $\begin{array}{l}\text { Well was reported abandoned and filled with } \\
\text { concrete. }\end{array}$ \\
\hline $14-5$ & 16 & $\begin{array}{l}\text { Unconsolidated material } 0-60 \\
\text { Bedrock } 60\end{array}$ & 4 -inch slotted casing inside of 6 -inch casing. \\
\hline $14-6$ & 16 & -- & $\begin{array}{l}\text { Water-level data for } 1962-69 \text { available under } \\
\text { "Well } 34 \text { " in Robison and others, } 1973 \text {. }\end{array}$ \\
\hline $14-7$ & 16 & -- & -- \\
\hline $14-8$ & 16 & -- & -- \\
\hline
\end{tabular}


Appendix B. Lithologic descriptions and remarks for wells on St. Thomas, U.S. Virgin Islands--Continued [--, indicates data not available]

\begin{tabular}{|c|c|c|c|}
\hline $\begin{array}{l}\text { Well } \\
\text { number }\end{array}$ & $\begin{array}{l}\text { Figure } \\
\text { number }\end{array}$ & $\begin{array}{l}\text { Lithologic description } \\
\text { and depth interval } \\
\text { (feet) }\end{array}$ & Remarks \\
\hline $14-9$ & 16 & -- & $\begin{array}{l}\text { Well drilled in } 1980^{\prime} \mathrm{s} \text {. Owner estimated daily } \\
\text { pumpage is } 3,000 \text { gallons. }\end{array}$ \\
\hline $14-10$ & 16 & $\begin{array}{l}\text { Unconsolidated material } 0-10 \\
\text { Bedrock } 10\end{array}$ & Very low yield reported. \\
\hline $14-11$ & 16 & -- & -- \\
\hline $14-12$ & 16 & $\begin{array}{l}\text { Unconsolidated material } 0-35 \\
\text { Bedrock } 35\end{array}$ & -- \\
\hline $14-13$ & 16 & $\begin{array}{l}\text { Unconsolidated material } 0-15 \\
\text { Bedrock } 15\end{array}$ & -- \\
\hline $15-1$ & 17 & $\begin{array}{l}\text { Unconsolidated material } 0-26 \\
\text { Bedrock } 26\end{array}$ & -- \\
\hline $15-2$ & 17 & -- & -- \\
\hline $15-3$ & 17 & $\begin{array}{l}\text { Unconsolidated material } 0-38 \\
\text { Bedrock } 38\end{array}$ & -- \\
\hline $15-4$ & 17 & -- & Well was drilled around 1970. \\
\hline $15-5$ & 17 & $\begin{array}{l}\text { Unconsolidated material } 0-19 \\
\text { Bedrock } 19\end{array}$ & -- \\
\hline $15-6$ & 17 & -- & -- \\
\hline $15-7$ & 17 & -- & -- \\
\hline $15-8$ & 17 & -- & -- \\
\hline $15-9$ & 17 & $\begin{array}{l}\text { Unconsolidated material } 0-25 \\
\text { Bedrock } 25\end{array}$ & -- \\
\hline $15-10$ & 17 & -- & -- \\
\hline $15-11$ & 17 & -- & -- \\
\hline $15-12$ & 17 & $\begin{array}{l}\text { Unconsolidated material } 0-16 \\
\text { Bedrock } 16\end{array}$ & -- \\
\hline $15-13$ & 17 & $\begin{array}{l}\text { Unconsolidated material } 0-18 \\
\text { Bedrock } 18\end{array}$ & -- \\
\hline $15-14$ & 17 & $\begin{array}{l}\text { Unconsolidated material } 0-30 \\
\text { Bedrock } 30\end{array}$ & -- \\
\hline $15-15$ & 17 & $\begin{array}{l}\text { Unconsolidated material } 0-55 \\
\text { Bedrock } 55\end{array}$ & $\begin{array}{l}\text { Estimated daily pumpage is } 4,000 \text { gallons and } \\
\text { reported to be } 8,000 \text { gallons during the wet } \\
\text { season. Well owned by Thomasville coop } \\
\text { Association. }\end{array}$ \\
\hline $15-16$ & 17 & $\begin{array}{l}\text { Unconsolidated material } 0-15 \\
\text { Bedrock } 15\end{array}$ & -- \\
\hline $15-17$ & 17 & $\begin{array}{l}\text { Unconsolidated material } 0-68 \\
\text { Bedrock } 68\end{array}$ & -- \\
\hline
\end{tabular}


Appendix B. Lithologic descriptions and remarks for wells on St. Thomas, U.S. Virgin Islands--Continued [--, indicates data not available]

\begin{tabular}{|c|c|c|c|}
\hline $\begin{array}{l}\text { Wel1 } \\
\text { number }\end{array}$ & $\begin{array}{l}\text { Figure } \\
\text { number }\end{array}$ & $\begin{array}{l}\text { Lithologic description } \\
\text { and depth interval } \\
\text { (feet) }\end{array}$ & Remarks \\
\hline $15-18$ & 17 & $\begin{array}{l}\text { Unconsolidated material } 0-18 \\
\text { Bedrock } 18\end{array}$ & -- \\
\hline $15-19$ & 17 & $\begin{array}{l}\text { Unconsolidated material } 0-40 \\
\text { Bedrock } 40\end{array}$ & -- \\
\hline $15-20$ & 17 & $\begin{array}{l}\text { Unconsolidated material } 0-35 \\
\text { Bedrock } 35\end{array}$ & -- \\
\hline $15-21$ & 17 & $\begin{array}{l}\text { Unconsolidated material } 0-45 \\
\text { Bedrock } 45\end{array}$ & -- \\
\hline $15-22$ & 17 & $\begin{array}{l}\text { Unconsolidated material } 0-45 \\
\text { Bedrock } 45\end{array}$ & -- \\
\hline $15-23$ & 17 & -- & Well drilled in 1986 or 1987. \\
\hline $15-24$ & 17 & $\begin{array}{l}\text { Unconsolidated material } 0-60 \\
\text { Bedrock } 60\end{array}$ & -- \\
\hline $15-25$ & 17 & -- & Well dug about 1900. \\
\hline $15-26$ & 17 & $\begin{array}{l}\text { Unconsolidated material } 0-40 \\
\text { Bedrock } 40\end{array}$ & - \\
\hline $15-27$ & 17 & -- & -- \\
\hline $15-28$ & 17 & -- & -- \\
\hline $15-29$ & 17 & -- & -- \\
\hline $15-30$ & 17 & $\begin{array}{l}\text { Unconsolidated material } 0-26 \\
\text { Bedrock } 26\end{array}$ & -- \\
\hline $15-31$ & 17 & -- & -- \\
\hline $15-32$ & 17 & $\begin{array}{l}\text { Unconsolidated material } 0-42 \\
\text { Bedrock } 42\end{array}$ & 4-inch casing inside of the 6-inch casing. \\
\hline $15-33$ & 17 & -- & -- \\
\hline $15-34$ & 17 & $\begin{array}{l}\text { Unconsolidated material } 0-28 \\
\text { Bedrock } 28\end{array}$ & $\begin{array}{l}\text { We11 owned by water and Power Authority. } \\
\text { Water-1evel data for } 1964-68 \text { available under } \\
\text { "Well 29" in Robison and others, } 1973 .\end{array}$ \\
\hline $15-35$ & 17 & $\cdots$ & -- \\
\hline $15-36$ & 17 & -- & $\begin{array}{l}\text { Wel1 was dug before } 1944 \text {. Water-level data for } \\
1958 \text { and } 1960-69 \text { available under "Well } 30 " \text { in } \\
\text { Robison and others, } 1973 \text {. }\end{array}$ \\
\hline $15-37$ & 17 & -- & -- \\
\hline $15-38$ & 17 & -- & -- \\
\hline $15-39$ & 17 & -- & -- \\
\hline $15-40$ & 17 & -- & -- \\
\hline $15-41$ & 17 & -- & -- \\
\hline $15-42$ & 17 & -- & -- \\
\hline
\end{tabular}


Appendix B. Lithologic descriptions and remarks for wells on St. Thomas, U.S. Virgin Islands--Continued

[--, indicates data not available]

$\begin{array}{ccc}\text { Wel1 } & \text { Figure } & \text { Lithologic description } \\ \text { number } & \text { number } & \text { Repth interval } \\ \text { (feet) }\end{array}$

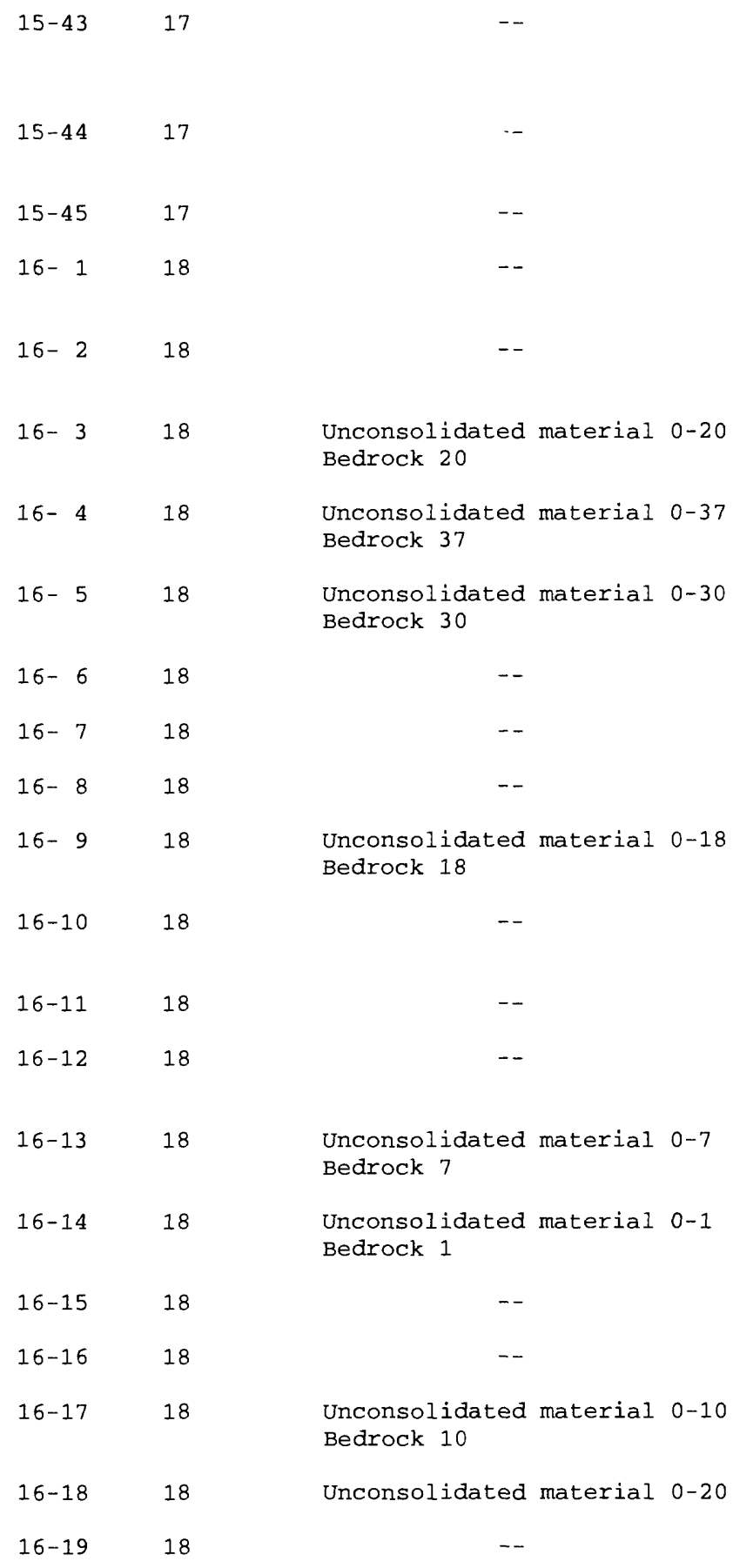

Well was constructed about 1970. Well casing is 3.5 feet by 2.5 feet. Well used in supply of 5 apartments.

Well reported drilled in early 1960's.

Well reported drilled about 1980 . Reported depth is 65-70 feet.

Water-level data for 1962-68 available under "We11 13" in Robison and others, 1973.

Reported 4-inch slotted casing from 0 to 200 feet.

Well dug in the early 1910's.

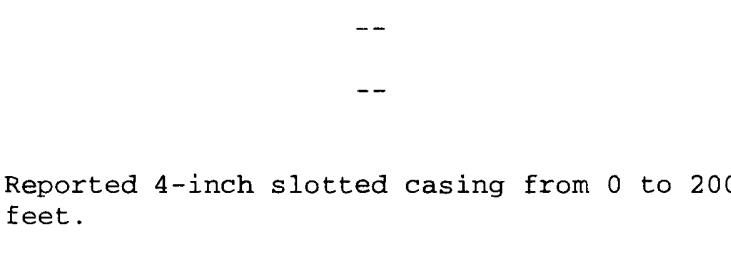

Well drilled in late 1970 's. Well may have caved in to $75-80$ feet.

Company president estimated daily pumpage is 3,000 gallons.

Well drilled about 1980 .

Sand filter on botton of casing. Maintenance personnel estimated daily pumpage is 40,000 gallons. 
Appendix B. Lithologic descriptions and remarks for wells on St. Thomas, U.S. Virgin Islands--Continued [--, indicates data not available]

\begin{tabular}{|c|c|c|c|}
\hline $\begin{array}{l}\text { Wel1 } \\
\text { number }\end{array}$ & $\begin{array}{l}\text { Figure } \\
\text { number }\end{array}$ & $\begin{array}{c}\text { Lithologic description } \\
\text { and depth interval } \\
\text { (feet) }\end{array}$ & Remarks \\
\hline $16-20$ & 18 & $\begin{array}{l}\text { Unconsolidated material } 0-28 \\
\text { Bedrock } 28\end{array}$ & $\begin{array}{l}\text { Well used in supply of condominiums. Well } \\
\text { owned by Cowper Bay west Condos. }\end{array}$ \\
\hline $16-21$ & 18 & $\begin{array}{l}\text { Unconsolidated material } 0-15 \\
\text { Bedrock } 15\end{array}$ & Well blocked at 37 feet, no water level. \\
\hline $16-22$ & 18 & $\begin{array}{l}\text { Unconsolidated material } 0-25 \\
\text { Bedrock } 25\end{array}$ & $\begin{array}{l}\text { Drilled in early } 1980 \text { 's. } 4 \text {-inch slotted casing } \\
\text { inside of } 6 \text {-inch casing to } 125 \text { feet. }\end{array}$ \\
\hline $16-23$ & 18 & -- & -- \\
\hline $16-24$ & 18 & -- & $\begin{array}{l}\text { Wel1 used in supply of condominiums. Well } \\
\text { owned by Anchorage Condo Association. }\end{array}$ \\
\hline
\end{tabular}

